US Army Corps

of Engineers $s_{\circledast}$

Engineer Research and

Development Center

\title{
Application of Laser Induced Breakdown Spectroscopy (LIBS) for Environmental, Chemical, and Biological Sensing
}

Holly VerMeulen, Jay L. Clausen, Ashley Mossell, Michael Morgan,

June 2021

Komi Messan, and Sam Beal 
The U.S. Army Engineer Research and Development Center (ERDC) solves the nation's toughest engineering and environmental challenges. ERDC develops innovative solutions in civil and military engineering, geospatial sciences, water resources, and environmental sciences for the Army, the Department of Defense, civilian agencies, and our nation's public good. Find out more at www.erdc.usace.army.mil.

To search for other technical reports published by ERDC, visit the ERDC online library at https://erdclibrary.on.worldcat.org/discovery. 


\section{Application of Laser Induced Breakdown Spectroscopy (LIBS) for Environmental, Chemical, and Biological Sensing}

Holly VerMeulen, Jay L. Clausen, Ashley Mossell, Michael Morgan, Komi Messan, and Sam Beal

Cold Regions Research and Engineering Laboratory

U.S. Army Engineer Research and Development Center

72 Lyme Road

Hanover, NH 03755

Final report

Approved for public release; distribution is unlimited.

Prepared for U.S. Army Corps of Engineers

Washington, DC 20134

Under Program Element Number 633734A, Project Number T15, Task 2 


\section{Preface}

This study was conducted for the US Army Corps of Engineers under Program Element Number 633734A, Project Number T15, Task 2. The technical monitor was Dr. Jay L. Clausen.

The work was performed by the Biogeochemical Sciences Branch (Mr. David Ringelberg, Acting Chief) of the Research and Engineering Division (Dr. George Calfas, Chief), U.S. Army Engineer Research and Development Center, Cold Regions Research and Engineering Laboratory (ERDC-ITL). At the time of publication, the Deputy Director of ERDCCRREL was Mr. David B. Ringelberg, and the Director was Dr. Joseph L. Corriveau.

This paper was originally presented at the SPIE 2019 Conference held in San Diego, CA on 11-15 August 2019 and published in the conference proceedings.

The authors would like to acknowledge the USACE ERDC T-15 Program for their contributions to this study.

The Commander of ERDC was COL Teresa A. Schlosser and the Director was Dr. David W. Pittman.

DISCLAIMER: The contents of this report are not to be used for advertising, publication, or promotional purposes. Citation of trade names does not constitute an official endorsement or approval of the use of such commercial products. All product names and trademarks cited are the property of their respective owners. The findings of this report are not to be construed as an official Department of the Army position unless so designated by other authorized documents. 


\title{
Application of laser induced breakdown spectroscopy (LIBS) for environmental, chemical, and biological sensing
}

\begin{abstract}
The Army is interested in sensors capable of characterizing/monitoring the environment (battlefield or military training ranges) at proximal distances. Recently, we evaluated laser induced breakdown spectroscopy (LIBS) systems (hand-held, proximal, and bench top) for the characterization of metals (antimony, copper, lead, tungsten, and zinc) in soils obtained from military training ranges. We then compared the results to findings obtained with standard field and laboratory instrumentation for metals analysis - X-ray Fluorescence (XRF) and Inductively Couple Plasma- Optical Emission Spectroscopy (ICP-OES).
\end{abstract}

\section{ACRONYM LIST}

ASI

ASTM

CONUS

CRREL

DOD

ERDC

GC/MS

HPLC

ICP-OES

LC/MS

LIBS

ng

OCONUS

ppm

USACE

XRF

Applied Spectra Inc.

American Society of Testing Materials

continental United States

Cold Regions Research and Engineering Laboratory

Department of Defense

Engineer Research and Development Center

gas chromatography/mass spectrometry

high-performance liquid chromatography

inductively-coupled plasma-optical emission spectrometry

liquid chromatography mass spectrometry

laser-induced breakdown spectroscopy

nanogram

outside continental United States

parts per million

U.S. Army Corps of Engineers

$\mathrm{x}$-ray fluorescence 


\section{INTRODUCTION}

Conventional analytical approaches to metal and organic analysis utilizes lab-based instruments such as X-ray Fluorescence (XRF), inductively coupled plasma- optical emission spectroscopy (ICP-OES), liquid chromatography mass spectrometry (LC/MS), high performance liquid chromatography (HPLC), or gas chromatography mass spectrometry (GC/MS). These methods involve collecting large amounts of sample, shipping the sample to a laboratory, sample preparation involving sieving, grinding, and subsampling for soils, extraction/digestion with an appropriate organic solvent, and analysis. Though high sensitivity and precision can be achieved through use of these instruments, extensive sample preparation is needed prior to analysis and results cannot be acquired in real-time in the field. In addition, prior knowledge about the site or sample is often needed. Samples must also be packaged and shipped back to the laboratory, which results in a much higher analysis cost and lengthier process overall. An emerging technology, laser induced breakdown spectroscopy (LIBS), promises the benefits associated with on-site proximal analysis; including rapid acquisition of sample results.

LIBS as an analytical measurement technology has existed since the beginning of the laser. It was recognized early that laser induced plasma could be used as a spectroscopic source for direct sampling and analysis. Benefits of LIBS include real time, simultaneous, multi-element chemical analysis without sample preparation. The basis of LIBS is ablation of a tiny portion of the sample using a laser beam to produce high temperature luminous plasma. A plasma spectrum consists of narrow atomic, ionic, and molecular lines of emitted light collected using standard optics, a spectrometer, and detector. LIBS is an atomic emission spectroscopic technique allowing for rapid elemental analysis of a wide variety of samples, even in austere environments ${ }^{1}$. A variety of optical spectrographs (Echelle, Czerny-Turner, and multi-channel) have been employed with LIBS. Chemometric analysis of the laser ablation spectra of a complex matrix like soil, sediment, or water containing an allogenic contaminant such as an explosive can be used to identify the contaminant from amongst the complex elemental and isotopic spectra of the soil material itself. In addition to elemental, molecular, and isotopic analysis, each LIBS spectrum is a unique spectral signature (equivalent to a barcode) for every material. This signature bears information about the chemical and physical properties of the sample. Although the LIBS spectra comprise mainly atomic emission lines, relative intensities and shapes of these lines depend upon the nature of the sample. Thus, LIBS can discriminate even compositionally similar molecular compounds. The focus to date has been on qualitative assessments to discriminate between different populations of data or to obtain a yes/no presence. Our work has focused on whether the LIBS technology can be utilized in a quantitative manner to determine the concentration of the material of interest.

LIBS instrumentation is relatively inexpensive, has been ruggedized for use in industrial settings and harsh environments, and is adaptable for direct or proximal analysis. The method is straightforward, inherently sensitive (low parts per million [ppm] range), requires little to no sample preparation, consumes only small amounts of sample, nanograms (ng), is capable of stratigraphic profiling, and is amenable to use in the field. LIBS has been successfully applied to the analysis of biomaterials $^{2}$, soil analysis ${ }^{3}$, explosives ${ }^{4}$, items of forensic interest ${ }^{5}$, and many other materials. A field portable LIBS

instrument, CHEMCAM, is currently gathering data at proximal distance of up to $3 \mathrm{~m}$ on Mars as part of the instrument suite on the Curiosity Rover ${ }^{6}$.

LIBS is a versatile analytical method applicable to many projects of interest to the DoD because of its potential for use in field operations. It is an advanced analytical measurement technology developed, modified, and refined over the last decade to provide a wide range of analytical capability. LIBS has many advantages over conventional analytical technologies such as XRF, ICP, GC, and HPLC (Table 1) ${ }^{7,8}$. One application of interest to the Army is in support of existing countermine sensor detection technologies. Whereas these technologies identify a target based on its shape, LIBS could be used to augment these technologies by providing chemical information, e.g. is there explosive residue on the soil surface associated with a buried object location. 
Table 1. LIBS and Comparable Analytical Technologies.

\begin{tabular}{|c|c|c|c|c|c|}
\hline & LIBS & $\mathrm{XRF}^{\mathrm{a}}$ & ICP-OES/MS ${ }^{b}$ & $\mathrm{GC} / \mathrm{MS}^{\mathrm{c}}$ & HPLC $^{\mathrm{d}}$ \\
\hline Field Portable & Yes & Yes & No & Yes & No \\
\hline Sample Preparation Required & No & No & Yes - 10's min. & $\begin{array}{c}\text { Yes }-10 \text { 's } \\
\text { min. }\end{array}$ & $\begin{array}{c}\text { Yes }-10 \mathrm{~s} \\
\text { min. }\end{array}$ \\
\hline Hazardous Chemical Usage & No & No & Yes & Yes & Yes \\
\hline Analysis Time & nano-sec. & min's. & 10's min. & 10's min. & 10 's min. \\
\hline Multi-element & Yes & Yes $^{1}$ & Yes $^{1}$ & No & No \\
\hline Molecular Compounds & Yes & No & No & Yes & $\mathrm{Yes}^{2}$ \\
\hline Isotopic and Speciation Capabilities & Yes & No & Yes & No & No \\
\hline Depth Profiling Capability & $\mathrm{Yes}^{3}$ & No & No & No & No \\
\hline O\&M Instrument Cost & No & No & Yes & Yes & Yes \\
\hline
\end{tabular}

${ }^{1}$ Not capable of analyzing light elements $-\mathrm{H}, \mathrm{He}, \mathrm{C}, \mathrm{N}, \mathrm{O}$

${ }^{2}$ Limited to energetic compounds

${ }^{3}$ Soil, biota, and other hard surfaces

${ }^{a} \mathrm{X}$-ray fluorescence

binductively coupled-optical emission spectroscopy/mass spectroscopy

'gas chromatography/mass spectrometry

dhigh-performance liquid-chromatography

LIBS analysis systems provide valuable chemical information on almost any type of sample. This is particularly true given the recent advances in laser and spectrometry manufacturing as well as chemometrics. The advent of broadband spectrometers for LIBS has extended applications into the molecular domain and provided enhanced capability by taking the ratio of the elemental peaks. Traditionally, LIBS has been confined to laboratory analysis due to the size and fragility of the instrument. Recent technological advances have focused on ruggedization and miniaturization of system components to bring this technology to the field for near real-time analysis ${ }^{7,8}$. Consequently, numerous manufacturers have developed commercially available, field-portable, LIBS systems in the past five years. All of these systems are limited to a qualitative output or are focused on matching an unknown material to a material in a reference library. However, the quantification sensitivity of these instruments is unknown and is the objective of this study.

\section{Objectives of study}

The goal for this study is to demonstrate the viability of LIBS for in-field analysis of interest to the Army. Ultimately, a methodology for proximal analysis is desired in support of environmental awareness and characterization without the need for prior knowledge of the materials or areas being surveyed. Lowering analytical costs through reduction of sample shipping, sample preparation, reduction in sample production waste, elimination of the need for hazardous chemicals from extraction/digestion are specific objectives to be demonstrated. Success will be based on the ability of the LIBS technology to detect the analytes of interest at the desired sensitivity. The U.S. Department of Defense (DoD) spends tens of millions of dollars on analytical monitoring and characterization activities and the widespread field implementation of LIBS could potentially save DoD millions of dollars in annual monitoring costs. In the current fiscal environment approaches to reduce operational and maintenance costs and investigation costs are extremely important to the sustainable operation of the DoD. We are also introduced in applications to support the warfighter.

\section{EXPERIMENTAL APPROACH}

Field-portable LIBS instruments underwent extensive laboratory validation and optimization through comparisons with CRRELs fixed-based laboratory J200 LIBS system as well as comparisons with XRF and ICP-OES for metals and HPLC for energetics. Comparisons were performed on soil samples using known analytical standards analyzed in triplicate on all instruments allowing for instrument optimization. Archived soil samples from a variety of military installations across 
CONUS and OCONUS, previously analyzed by ERDC with XRF and ICP-OES, will then be analyzed with the fieldportable LIBS systems.

Prior to analysis, all soils were air dried, sieved to $<2 \mathrm{~m}$ particle size, milled for 5 minutes, and then pressed into pellets. Pellets were made using a die and applying a load of 2 tons for 3 minutes.

\subsection{XRF}

A subsample was taken from each sample and placed in a clear plastic bag. Samples were then analyzed with an Innov-X alpha series XRF by placing the instrument in direct contact with the sample utilizing a 2 minute counting interval.

\subsection{ICP-OES}

One gram of each sample was digested with a mixture of $2.5 \mathrm{~mL}$ nitric acid and $10 \mathrm{~mL}$ hydrochloric acid at $95{ }^{\circ} \mathrm{C}$ for 4 hours. Digests were allowed to cool and then diluted with MilliQ water to a final concentration of $2 \%$ and $5 \%$ nitric acid and hydrochloric acid respectively. Samples were digested in triplicate. Aliquots from these digestates were then analyzed with a Thermo Scientific iCAP 6000 Series ICP-OES followed US EPA Method 3050 procedures.

\subsection{LIBS}

Samples analyzed with the Applied Spectra J200 LIBS utilized a $266 \mathrm{~nm}$ laser, with a $25 \mathrm{~mJ}$ nominal maximum energy output, 250 nanosecond spectrometer delay, 9 integrated shots within a $3 \times 3$ grid and quantified based on calibration completed with NIST certified reference soils. NIST reference soils of known concentrations were combined with $\mathrm{KBr}$ and ground with mortar and pestle to dilute the material to concentrations between 0 and 100,000 ppm. These mixtures were then formed into $6 \mathrm{~mm}$ diameter pellets using a die at 2850 psi for 3 minutes. Four standards were used to develop calibration curves for quantification of the LIBS results: antimony, lead, tungsten, and zinc. A single variable linear approach was employed to develop calibration curves for each element from the intensity of the peak at that wavelength. Lead was quantified at the $405.768 \mathrm{~nm}$ wavelength, antimony at $276.956 \mathrm{~nm}$, tungsten at $401.648 \mathrm{~nm}$, and zinc at $202.535 \mathrm{~nm}$. Analysis of samples with a hand-held LIBs instrument is yet to commence so the focus of our work to date has been on the performance of the bench top LIBS to conventional detection technologies such as ICP-OES and XRF.

\section{RESULTS AND DISCUSSION}

Calibration of standard reference soils with the bench top LIBS system was conducted using a single variable linear approach (Figure 1). Four elements were used for the calibration: antimony, lead, zinc, and tungsten. Both lead and tungsten demonstrated a very linear relationship with peak intensity, having correlation coefficients above 0.89 , followed by zinc (0.81) and antimony (0.66) respectively. However, the concentrations calculated with these calibration curve were not in good agreement with concentrations found using the more conventional analytical techniques (Tables 2 and A1). Trends varied both between sites as well as among analytes (Table A1). This suggests that a single variable linear approach to calibration may not be as accurate as a multivariate approach to calibration. It has been supported that a multivariate approach that takes into account multiple emission lines for a single element, coupled with chemometric methods of analysis, are more robust ${ }^{9}$. This type of model is currently being developed for the data collected in this study. 


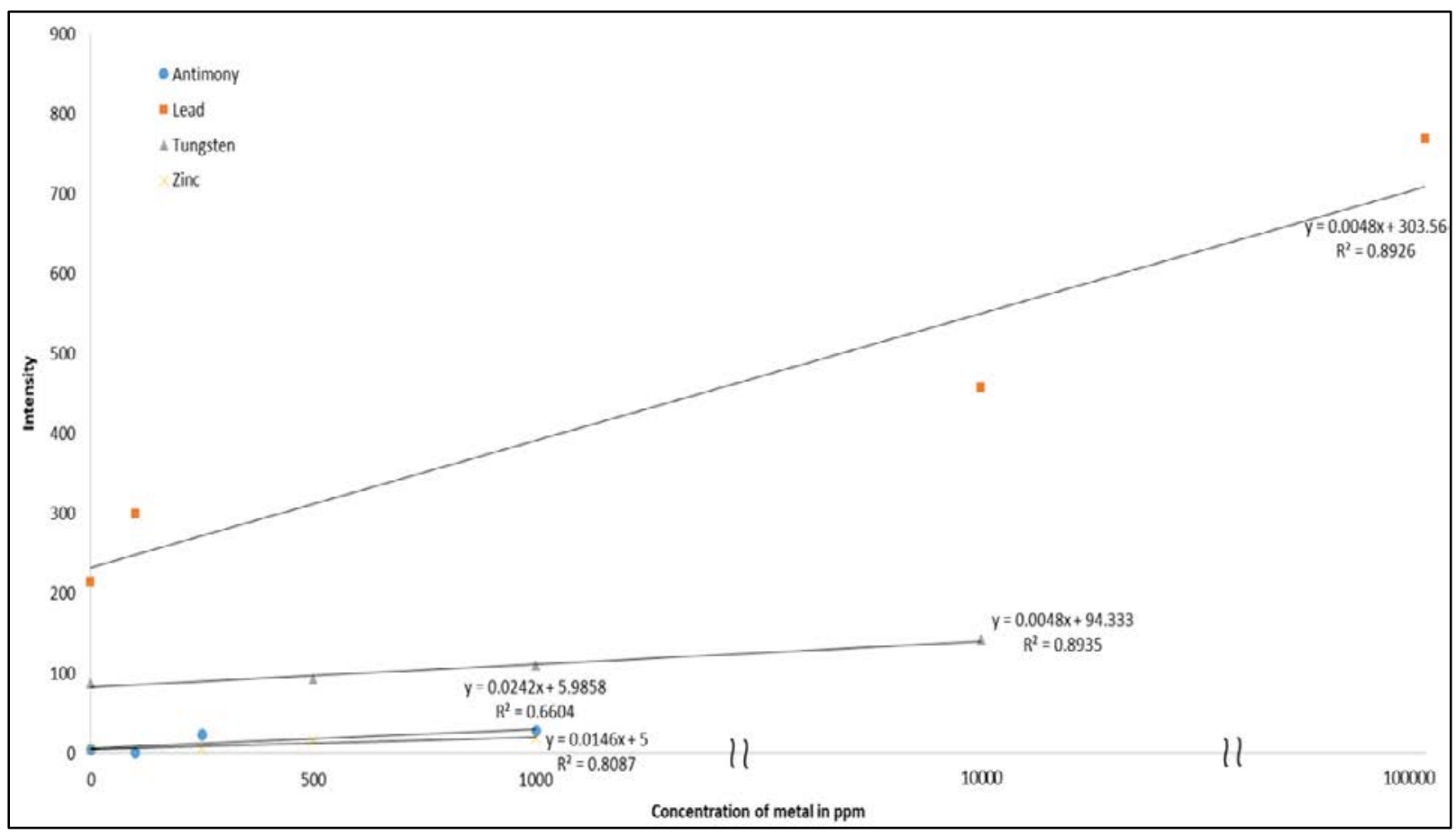

Figure 1. Single variable linear calibration of select metals (antimony, lead, tungsten and zinc) with Applied Spectra J200 LIBS bench-top system

Because of the high variability between LIBS systems, the calculated concentrations obtained using the bench top LIBS system were compared with XRF and ICP values for the same samples to determine if a relationship exists between the data sets that could be used to predict a theoretical LIBS value. This theoretical value could then be used to test for accuracy in the experimentally obtained LIBS concentrations. A Spearman correlation was used because the assumption of normality for a Pearson correlation was not met. When comparing LIBS concentrations to ICP values, an exponential function results in a correlation coefficient of 0.35 (Figure 2), which is higher than that obtained using a linear model $(0.21)$. The $\mathrm{R}^{2}$ value is relatively low between these sets of data due to the large spread within the datasets. A modified exponential function was also used to compare the same LIBS data with XRF concentrations for the soils; resulting in a correlation coefficient of 0.58 (Figure 3). The exponential function provides the same R-square when linear relationship is observed. 


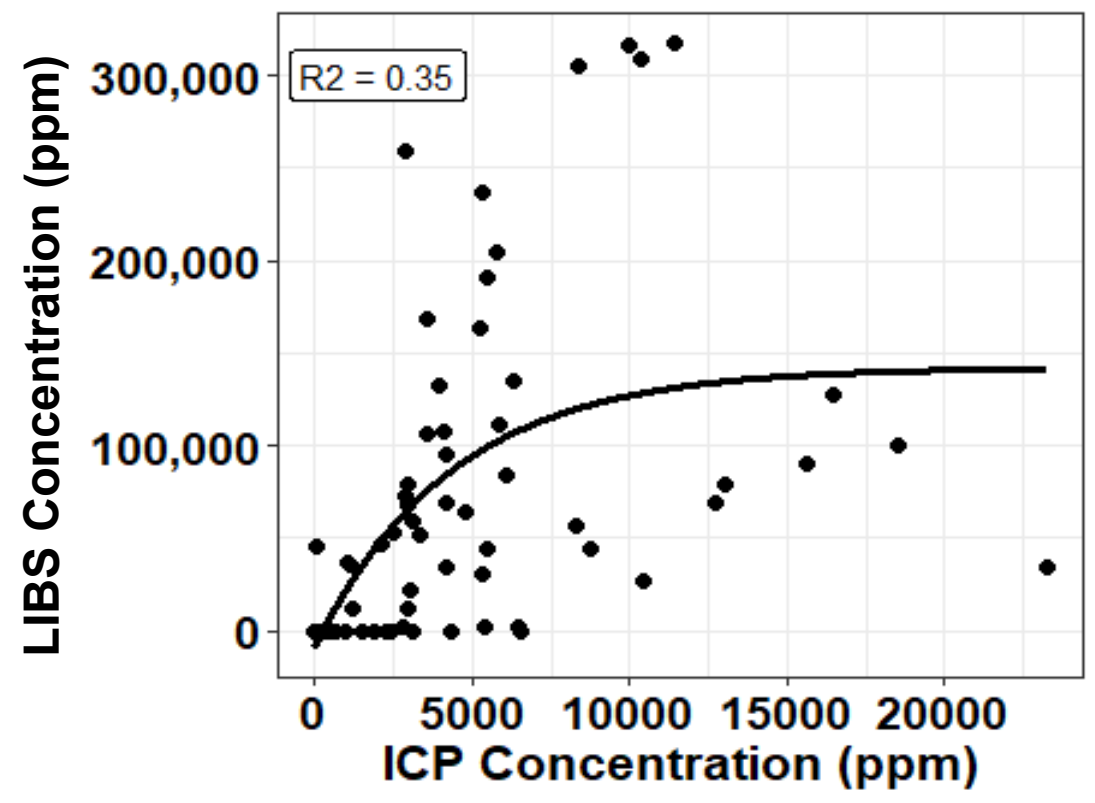

Figure 2. Exponential relationship between lead concentrations found with bench top LIBS and ICP systems.

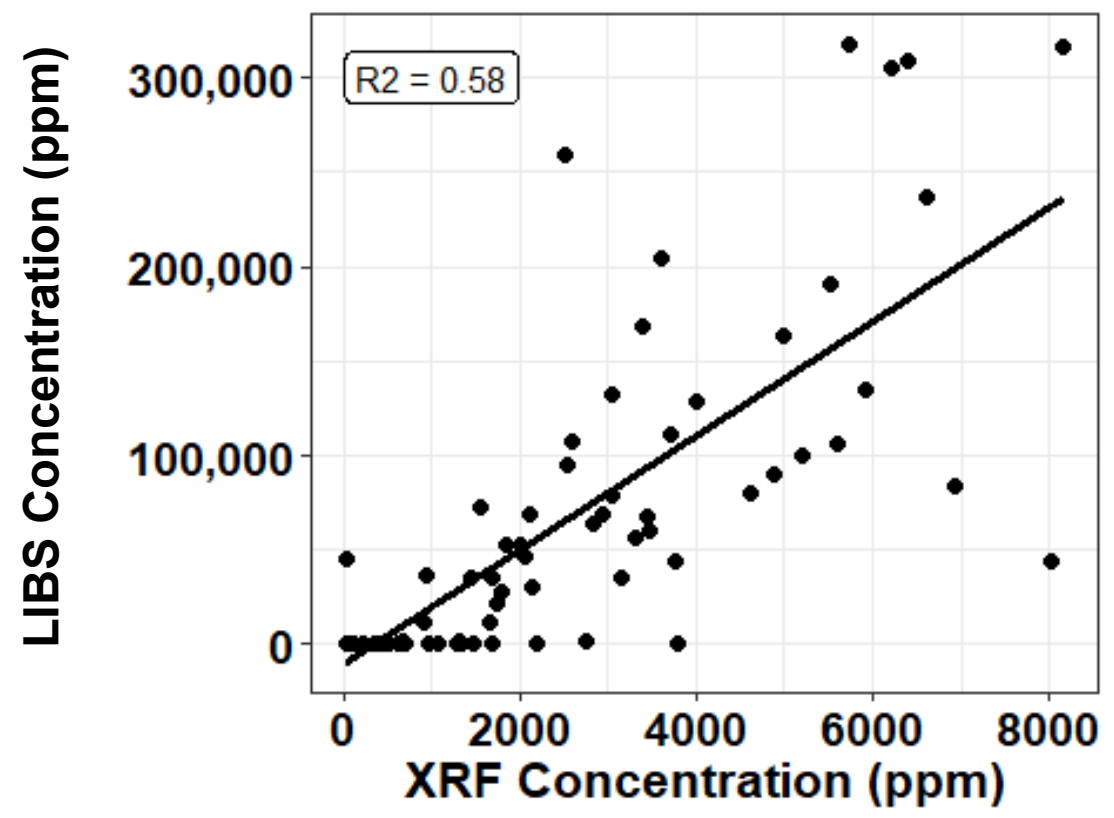

Figure 3. Exponential relationship between lead concentrations found with bench top LIBS and XRF. 
From this relationship, an exponential model was generated (Equation 1) that can be used to predict theoretical LIBS concentrations from experimentally obtained ICP or XRF values. This value can then serve as an accepted value to assess accuracy of lab-based results.

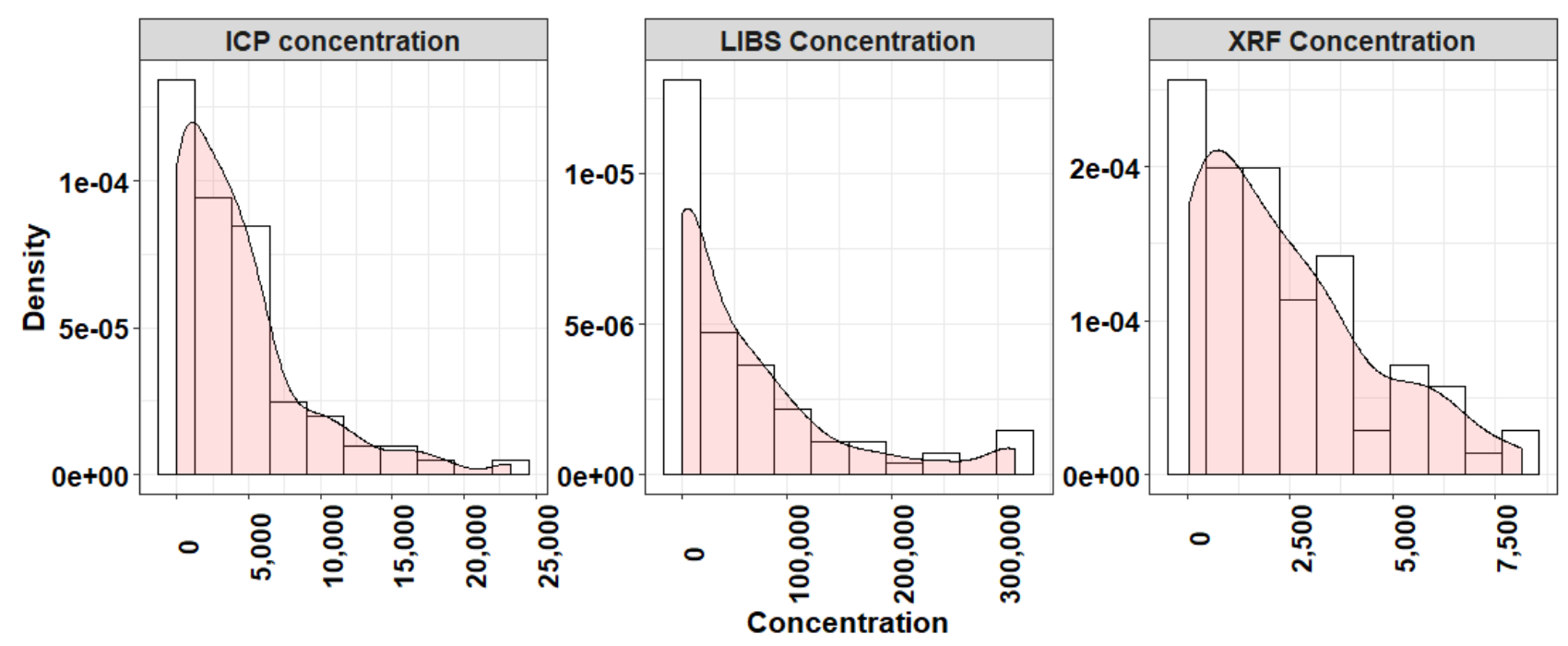

Figure 4. Histogram with density plot of for the ICP, LIBS, and XRF concentration values.

$C_{L I B S}=\mathrm{c}+(d-c)\left(1-e^{\frac{-x}{e}}\right)$

(Equation 1)

where $\quad x=\mathrm{XRF} / \mathrm{ICP}$ Concentration of a given material

$c=$ lower limit when LIBS concentration is 0

$d=$ upper limit

$e=$ steepness of the increase as $\mathrm{x}$ changes

Given that the none of the concentration density values follow a normal distribution (Figure 4), a Spearman Rank Correlation was used to determine if the relationship between ICP and XRF concentrations and LIBS concentrations was statistically significant (Table 2 ). The variable $\rho$ denotes the spearman rank correlation coefficient with the corresponding $P$-value of the test. In both cases, the relationship between LIBS and the compared technology were statistically significant at a confidence level or 0.001 . This suggests that the model can be used to predict theoretical LIBS concentrations.

Table 2. Spearman Rank Correlation between LIBS and comparable analytical technologies

\begin{tabular}{|c|c|c|}
\hline Concentration values & $\boldsymbol{\rho}$ & P-value \\
\hline LIBS and ICP & 0.72 & $<0.0001$ \\
\hline LIBS and XRF & 0.83 & $<0.0001$ \\
\hline
\end{tabular}

\section{CONCLUSIONS}

LIBS offers several potential advantages over conventional analytical approaches to inorganic analysis for field screening, characterization, and routine monitoring applications. Calibration of standard reference soils for LIBS using a single variable linear approach demonstrated a linear relationship between metal concentration and peak intensity at the chosen wavelength for each analyte. Tungsten showed the most linear relationship, followed by lead, zinc, and antimony. 
However, these results did not agree well with the other analysis methods, suggesting low precision in the measurements or calibration technique. There is, however, a relationship between these other techniques and the concentrations obtained using the bench top LIBS system that is best described using an exponential function in both cases. This function can be used to determine theoretical LIBS values from concentrations obtained with ICP or XRF and help to verify the accuracy of LIBS data collected experimentally.

\section{ACKNOWLEDGMENTS}

The authors would like to acknowledge the USACE ERDC T-15 Program for their contributions to this study.

\section{REFERENCES}

[1] D.A. Cremers and L.J. Radziemski, Handbook of Laser-Induced Breakdown Spectroscopy, John Wiley \& Sons, 2006; Laser-Induced Breakdown Spectroscopy, J.P Singh and S.N. Thakur, Eds., Elsevier Science Publishing, 2007; Laser Induced Breakdown Spectroscopy, A.W. Miziolek, V. Palleschi and I. Schechter, Eds., Cambridge University Press, 2008; R. Noll, Laser-Induced Breakdown Spectroscopy: Fundamentals and Applications, Springer, 2012.

[2] J.L. Gottfried, F.C. De Lucia, Jr, C.A. Munson, A.W. Miziolek, "Standoff detection of chemical and biological threats using laser-induced breakdown spectroscopy," Appl. Spectrosc. 2008, 62, 353-63.

[3] S.C. Jantzi, J. R. Almirall, "Characterization and forensic analysis of soil samples using laser-induced breakdown spectroscopy (LIBS),” Anal. Bioanal. Chem. 2011, 400, 3341-3351.

[4] J.L. Gottfried, F.C. De Lucia, Jr., C.A. Munson, A.W. Miziolek, "Laser-induced breakdown spectroscopy for explosive residue detection: a review of the challenges, recent advances, and future prospects," Anal. Bioanal. Chem. 2009, 395, 283-300.

[5] E.M. Cahoon, J.R. Almirall, "Wavelength dependence on the forensic analysis of glass by nanosecond $266 \mathrm{~nm}$ and $1064 \mathrm{~nm}$ laser induced breakdown spectroscopy," Appl. Optics 2010, 49, C49-C57.

[6] "Rover's Laser Instrument Zaps First Martian Rock," Accessed 24 September 2012, http://www.jpl.nasa.gov/news/news.php?release=2012-2488.

[7] J. Rakovsky, P. Cermak, O. Musset, and P. Veis, A review of the development of portable laser induced breakdown spectroscopy and its applications, Spectrochimica Acta Part B, 101:269-287. (2014).

[8] F.J. Fortes and J.J. Laserna, The development of fieldable laser-induced breakdown spectrometer: No limits on the horizon, Spectrochimica Acta Part B, 65:975-990, (2010).

[9] Labbe, N., et al., Extraction of information from laser-induced breakdown spectroscopy spectroscopy spectral data by multivariate analysis. Appl Opt, 2008. 47(31). 


\section{APPENDIX A}

Table A1. Concentrations of lead, antimony, tungsten, and zinc in military soils measured using ICP-OES, XRF, and bench top LIBS.

\begin{tabular}{|c|c|c|c|c|c|c|c|c|c|c|c|c|c|}
\hline & & \multicolumn{3}{|c|}{ Lead } & \multicolumn{3}{|c|}{ Tungsten } & \multicolumn{3}{|c|}{ Antimony } & \multicolumn{3}{|c|}{ Zinc } \\
\hline $\begin{array}{c}\text { Sample } \\
\text { ID }\end{array}$ & Location & ICP & XRF & LIBS & ICP & XRF & LIBS & ICP & XRF & $\begin{array}{c}\text { LIB } \\
\text { S }\end{array}$ & ICP & XRF & LIBS \\
\hline B01 & Ft. Benning & $\begin{array}{c}6056 . \\
90 \\
\end{array}$ & $\begin{array}{c}6928 . \\
00 \\
\end{array}$ & $\begin{array}{c}84096 \\
.30 \\
\end{array}$ & $\begin{array}{c}85.5 \\
0 \\
\end{array}$ & - & 0.00 & 97.30 & $\begin{array}{c}122.0 \\
0 \\
\end{array}$ & $\begin{array}{c}2080 \\
.48 \\
\end{array}$ & 44.20 & 78.00 & 0.00 \\
\hline B02 & Ft. Benning & $\begin{array}{l}1855 \\
1.50 \\
\end{array}$ & $\begin{array}{c}5188 . \\
00\end{array}$ & $\begin{array}{c}99675 \\
.00\end{array}$ & $\begin{array}{c}92.7 \\
0\end{array}$ & - & 0.00 & $\begin{array}{c}279.7 \\
0\end{array}$ & - & $\begin{array}{c}1088 \\
.74\end{array}$ & 70.60 & 70.00 & 0.00 \\
\hline B03 & Ft. Benning & $\begin{array}{c}6335 . \\
20\end{array}$ & $\begin{array}{c}5929 . \\
00\end{array}$ & $\begin{array}{c}13486 \\
0.20\end{array}$ & $\begin{array}{c}87.4 \\
0\end{array}$ & - & 0.00 & $\begin{array}{c}113.4 \\
0\end{array}$ & - & $\begin{array}{c}1221 \\
.89\end{array}$ & 42.60 & 78.00 & 0.00 \\
\hline B04 & Ft. Benning & $\begin{array}{l}1648 \\
4.20 \\
\end{array}$ & $\begin{array}{c}3999 . \\
00 \\
\end{array}$ & $\begin{array}{c}12798 \\
5.20 \\
\end{array}$ & $\begin{array}{c}101 . \\
00\end{array}$ & - & 0.00 & $\begin{array}{c}239.0 \\
0 \\
\end{array}$ & - & $\begin{array}{c}1336 \\
.68 \\
\end{array}$ & 46.90 & 68.00 & 0.00 \\
\hline B05 & Ft. Benning & $\begin{array}{l}1558 \\
4.90 \\
\end{array}$ & $\begin{array}{c}4887 . \\
00\end{array}$ & $\begin{array}{c}90300 \\
.00\end{array}$ & $\begin{array}{c}116 . \\
50\end{array}$ & - & 0.00 & $\begin{array}{c}642.4 \\
0\end{array}$ & 85.00 & $\begin{array}{c}69.4 \\
6 \\
\end{array}$ & $\begin{array}{c}143.5 \\
0\end{array}$ & 62.00 & 0.00 \\
\hline B08 & Ft. Benning & $\begin{array}{c}5848 . \\
70 \\
\end{array}$ & $\begin{array}{c}3710 . \\
00\end{array}$ & $\begin{array}{c}11115 \\
6.50 \\
\end{array}$ & $\begin{array}{c}120 . \\
90 \\
\end{array}$ & $\begin{array}{c}201.0 \\
0 \\
\end{array}$ & 0.00 & 77.70 & 63.00 & $\begin{array}{c}1740 \\
.72 \\
\end{array}$ & 53.70 & 56.00 & 0.00 \\
\hline $\mathrm{B} 10$ & Ft. Benning & $\begin{array}{c}3355 . \\
00\end{array}$ & $\begin{array}{c}2008 . \\
00\end{array}$ & $\begin{array}{c}52360 \\
.19\end{array}$ & $\begin{array}{c}85.1 \\
0\end{array}$ & $\begin{array}{c}142.0 \\
0\end{array}$ & 0.00 & 45.30 & - & $\begin{array}{c}2796 \\
.73\end{array}$ & 28.20 & 45.00 & 0.00 \\
\hline B11 & Ft. Benning & $\begin{array}{l}2323 \\
7.80\end{array}$ & $\begin{array}{c}1456 . \\
00\end{array}$ & $\begin{array}{c}34975 \\
.93\end{array}$ & $\begin{array}{c}44.0 \\
0\end{array}$ & $\begin{array}{c}228.0 \\
0\end{array}$ & 0.00 & $\begin{array}{c}533.8 \\
0\end{array}$ & - & $\begin{array}{c}1107 \\
.11\end{array}$ & $\begin{array}{c}153.0 \\
0\end{array}$ & 44.00 & 0.00 \\
\hline B12 & Ft. Benning & $\begin{array}{c}6530 . \\
60\end{array}$ & $\begin{array}{c}228.0 \\
0\end{array}$ & 0.00 & $\begin{array}{c}36.9 \\
0\end{array}$ & - & 0.00 & $\begin{array}{c}117.7 \\
0\end{array}$ & - & $\begin{array}{c}1281 \\
.58\end{array}$ & 12.40 & 17.00 & $\begin{array}{c}799.0 \\
9\end{array}$ \\
\hline B13 & Ft. Benning & $\begin{array}{c}1532 . \\
40 \\
\end{array}$ & $\begin{array}{c}224.0 \\
0 \\
\end{array}$ & 0.00 & - & - & 0.00 & 20.40 & - & $\begin{array}{c}1561 \\
.65 \\
\end{array}$ & 10.80 & 21.00 & 0.00 \\
\hline B14 & Ft. Benning & $\begin{array}{c}6454 . \\
70\end{array}$ & $\begin{array}{c}2751 . \\
00\end{array}$ & $\begin{array}{c}2175 . \\
00\end{array}$ & $\begin{array}{c}110 . \\
40\end{array}$ & - & 0.00 & $\begin{array}{c}120.6 \\
0\end{array}$ & - & $\begin{array}{c}- \\
224 . \\
39\end{array}$ & 32.40 & 53.00 & 0.00 \\
\hline B15 & Ft. Benning & $\begin{array}{c}4219 . \\
50 \\
\end{array}$ & $\begin{array}{c}2547 . \\
00\end{array}$ & $\begin{array}{c}95300 \\
.00 \\
\end{array}$ & $\begin{array}{c}105 . \\
90 \\
\end{array}$ & $\begin{array}{c}134.0 \\
0 \\
\end{array}$ & 0.00 & 78.90 & - & 0.00 & 73.70 & 42.00 & 0.00 \\
\hline B16 & Ft. Benning & $\begin{array}{c}2872 . \\
90\end{array}$ & $\begin{array}{c}1548 . \\
00\end{array}$ & $\begin{array}{c}72545 \\
.37\end{array}$ & $\begin{array}{c}16.6 \\
0\end{array}$ & - & 0.00 & 56.20 & - & $\begin{array}{c}588 . \\
28\end{array}$ & 23.90 & 35.00 & 0.00 \\
\hline B17 & Ft. Benning & $\begin{array}{c}1891 . \\
90\end{array}$ & $\begin{array}{c}1689 . \\
00\end{array}$ & 0.00 & $\begin{array}{c}37.0 \\
0 \\
\end{array}$ & - & 0.00 & 36.50 & - & 0.00 & 25.00 & 45.00 & 0.00 \\
\hline B18 & Ft. Benning & $\begin{array}{c}283.7 \\
0 \\
\end{array}$ & $\begin{array}{c}614.0 \\
0 \\
\end{array}$ & 0.00 & - & - & 0.00 & 5.00 & - & $\begin{array}{c}1345 \\
.86 \\
\end{array}$ & 19.40 & 47.00 & 0.00 \\
\hline B19 & Ft. Benning & $\begin{array}{c}265.8 \\
0\end{array}$ & $\begin{array}{c}473.0 \\
0\end{array}$ & 0.00 & - & - & 0.00 & 6.60 & - & $\begin{array}{c}1456 \\
.05\end{array}$ & 22.80 & 39.00 & $\begin{array}{c}464.2 \\
3\end{array}$ \\
\hline $\mathrm{B} 20$ & Ft. Benning & 62.50 & 85.00 & 0.00 & - & - & 0.00 & 7.70 & - & $\begin{array}{c}1763 \\
.67 \\
\end{array}$ & 29.70 & 37.00 & 0.00 \\
\hline B21 & Ft. Benning & $\begin{array}{c}105.2 \\
0\end{array}$ & $\begin{array}{c}119.0 \\
0\end{array}$ & 0.00 & - & - & 0.00 & 6.10 & - & $\begin{array}{c}1965 \\
.69 \\
\end{array}$ & 16.30 & 27.00 & 0.00 \\
\hline $\mathrm{B} 22$ & Ft. Benning & $\begin{array}{c}1304 \\
4.70 \\
\end{array}$ & $\begin{array}{c}3036 . \\
00\end{array}$ & $\begin{array}{c}78610 \\
.19 \\
\end{array}$ & $\begin{array}{c}52.5 \\
0 \\
\end{array}$ & - & 0.00 & $\begin{array}{c}266.9 \\
0 \\
\end{array}$ & - & $\begin{array}{c}1543 \\
.29 \\
\end{array}$ & 26.30 & 47.00 & 0.00 \\
\hline $\mathrm{B} 23$ & Ft. Benning & $\begin{array}{c}2097 . \\
90\end{array}$ & $\begin{array}{c}2047 . \\
00\end{array}$ & $\begin{array}{c}47059 \\
.26\end{array}$ & $\begin{array}{c}37.6 \\
0\end{array}$ & - & 0.00 & 18.90 & - & $\begin{array}{c}1249 \\
.44\end{array}$ & 23.70 & 46.00 & 0.00 \\
\hline B24 & Ft. Benning & $\begin{array}{c}3989 . \\
00\end{array}$ & $\begin{array}{c}3034 . \\
00\end{array}$ & $\begin{array}{c}13231 \\
3.90 \\
\end{array}$ & $\begin{array}{c}47.0 \\
0 \\
\end{array}$ & - & 0.00 & 68.60 & - & $\begin{array}{c}914 . \\
27 \\
\end{array}$ & 60.30 & 51.00 & 0.00 \\
\hline $\mathrm{B} 25$ & Ft. Benning & $\begin{array}{c}1880 . \\
80\end{array}$ & $\begin{array}{c}3781 . \\
00\end{array}$ & 0.00 & $\begin{array}{c}41.2 \\
0\end{array}$ & - & 0.00 & 33.30 & - & $\begin{array}{c}941 . \\
82\end{array}$ & 21.60 & 65.00 & 0.00 \\
\hline B26 & Ft. Benning & $\begin{array}{c}2454 . \\
80\end{array}$ & $\begin{array}{c}2187 . \\
00\end{array}$ & 0.00 & $\begin{array}{c}77.0 \\
0\end{array}$ & - & 0.00 & 41.20 & - & $\begin{array}{c}124 . \\
55\end{array}$ & $\begin{array}{c}251.4 \\
0\end{array}$ & 42.00 & 91.32 \\
\hline B27 & Ft. Benning & $\begin{array}{c}196.9 \\
0\end{array}$ & $\begin{array}{c}417.0 \\
0\end{array}$ & 0.00 & - & - & 0.00 & - & - & $\begin{array}{c}400 . \\
04\end{array}$ & 4.60 & 17.00 & 0.00 \\
\hline B28 & Ft. Benning & $\begin{array}{c}650.6 \\
0\end{array}$ & $\begin{array}{c}350.0 \\
0\end{array}$ & 0.00 & - & - & 0.00 & 5.70 & - & $\begin{array}{c}693 . \\
88\end{array}$ & 5.40 & 18.00 & 0.00 \\
\hline
\end{tabular}




\begin{tabular}{|c|c|c|c|c|c|c|c|c|c|c|c|c|c|}
\hline B29 & Ft. Benning & $\begin{array}{c}3140 . \\
40\end{array}$ & $\begin{array}{c}1341 . \\
00\end{array}$ & 0.00 & $\begin{array}{c}55.5 \\
0\end{array}$ & - & 0.00 & 37.70 & - & $\begin{array}{c}1901 \\
.41\end{array}$ & 25.10 & 28.00 & 0.00 \\
\hline $\mathrm{B} 30$ & Ft. Benning & $\begin{array}{c}4320 . \\
20 \\
\end{array}$ & $\begin{array}{c}1273 . \\
00 \\
\end{array}$ & 0.00 & $\begin{array}{c}111 . \\
50\end{array}$ & - & 0.00 & 89.00 & - & $\begin{array}{c}1561 \\
.65 \\
\end{array}$ & 21.20 & 41.00 & 0.00 \\
\hline B31 & Ft. Benning & $\begin{array}{c}2245 . \\
10 \\
\end{array}$ & $\begin{array}{c}698.0 \\
0 \\
\end{array}$ & 0.00 & - & - & 0.00 & 42.10 & - & $\begin{array}{c}827 . \\
03 \\
\end{array}$ & 4.30 & 22.00 & $\begin{array}{c}639.2 \\
7 \\
\end{array}$ \\
\hline B32 & Ft. Benning & $\begin{array}{c}376.8 \\
0 \\
\end{array}$ & $\begin{array}{c}689.0 \\
0 \\
\end{array}$ & 0.00 & - & - & 0.00 & 20.00 & - & $\begin{array}{c}354 . \\
12 \\
\end{array}$ & 5.10 & 14.00 & $\begin{array}{c}646.8 \\
8 \\
\end{array}$ \\
\hline B33 & Ft. Benning & $\begin{array}{c}563.3 \\
0\end{array}$ & $\begin{array}{c}1472 . \\
00\end{array}$ & 0.00 & 5.80 & - & 0.00 & 7.30 & - & $\begin{array}{c}2737 \\
.04\end{array}$ & 12.00 & 39.00 & 0.00 \\
\hline B34 & Ft. Benning & $\begin{array}{c}5467 . \\
20 \\
\end{array}$ & $\begin{array}{c}3769 . \\
00 \\
\end{array}$ & $\begin{array}{c}44258 \\
.33 \\
\end{array}$ & $\begin{array}{c}79.2 \\
0 \\
\end{array}$ & - & 0.00 & 97.70 & 83.00 & $\begin{array}{c}1538 \\
.70 \\
\end{array}$ & 29.90 & 60.00 & 0.00 \\
\hline B35 & Ft. Benning & $\begin{array}{c}4201 . \\
30\end{array}$ & $\begin{array}{c}3161 . \\
00\end{array}$ & $\begin{array}{c}34767 \\
.59 \\
\end{array}$ & 5.70 & - & 0.00 & 32.30 & - & $\begin{array}{c}1093 \\
.33\end{array}$ & 30.80 & 41.00 & 0.00 \\
\hline B36 & Ft. Benning & $\begin{array}{c}5386 . \\
80\end{array}$ & $\begin{array}{c}666.0 \\
0\end{array}$ & $\begin{array}{c}1735 . \\
19\end{array}$ & $\begin{array}{c}54.9 \\
0 \\
\end{array}$ & - & 0.00 & 86.50 & - & $\begin{array}{c}681 \\
.03\end{array}$ & 24.90 & 23.00 & $\begin{array}{c}1118 . \\
72 \\
\end{array}$ \\
\hline B37 & Ft. Benning & $\begin{array}{c}989.8 \\
0\end{array}$ & $\begin{array}{c}961.0 \\
0\end{array}$ & 0.00 & $\begin{array}{c}12.2 \\
0\end{array}$ & - & 0.00 & 15.60 & - & $\begin{array}{c}726 . \\
02\end{array}$ & 17.40 & 28.00 & 0.00 \\
\hline B38 & Ft. Benning & $\begin{array}{c}5318 . \\
20 \\
\end{array}$ & $\begin{array}{c}2150 . \\
00\end{array}$ & $\begin{array}{c}30925 \\
.00 \\
\end{array}$ & 9.60 & - & 0.00 & 74.70 & 71.00 & $\begin{array}{c}1364 \\
.22 \\
\end{array}$ & $\begin{array}{c}127.6 \\
0 \\
\end{array}$ & 48.00 & 0.00 \\
\hline B39 & Ft. Benning & $\begin{array}{c}3034 . \\
30\end{array}$ & $\begin{array}{c}1752 . \\
00\end{array}$ & $\begin{array}{c}21503 \\
.70\end{array}$ & - & - & 0.00 & 25.80 & 68.00 & $\begin{array}{c}721 . \\
43\end{array}$ & $\begin{array}{c}1062 . \\
50\end{array}$ & 44.00 & $\begin{array}{c}616.4 \\
4\end{array}$ \\
\hline B40 & Ft. Benning & $\begin{array}{l}1271 \\
0.70\end{array}$ & $\begin{array}{c}2934 . \\
00\end{array}$ & $\begin{array}{c}68911 \\
.11\end{array}$ & $\begin{array}{c}304 . \\
60\end{array}$ & - & 0.00 & $\begin{array}{c}228.8 \\
0\end{array}$ & - & $\begin{array}{c}436 . \\
77\end{array}$ & 34.00 & 55.00 & 0.00 \\
\hline B41 & Ft. Benning & $\begin{array}{c}4090 . \\
60 \\
\end{array}$ & $\begin{array}{c}2591 . \\
00\end{array}$ & $\begin{array}{c}10768 \\
4.30\end{array}$ & $\begin{array}{c}135 . \\
40\end{array}$ & - & 0.00 & 71.30 & 60.00 & $\begin{array}{c}1524 \\
.92\end{array}$ & 29.70 & 44.00 & $\begin{array}{c}251.1 \\
4\end{array}$ \\
\hline B42 & Ft. Benning & $\begin{array}{c}2982 . \\
10\end{array}$ & $\begin{array}{c}4602 . \\
00\end{array}$ & $\begin{array}{c}79698 \\
.15 \\
\end{array}$ & $\begin{array}{c}18.0 \\
0 \\
\end{array}$ & - & 0.00 & 32.40 & 64.00 & $\begin{array}{c}1217 \\
.30 \\
\end{array}$ & 29.00 & 64.00 & 0.00 \\
\hline B43 & Ft. Benning & $\begin{array}{c}4212 . \\
80\end{array}$ & $\begin{array}{c}2103 . \\
00\end{array}$ & $\begin{array}{c}68702 \\
.78\end{array}$ & 4.40 & - & 0.00 & 29.60 & - & $\begin{array}{c}1084 \\
.15\end{array}$ & 40.40 & 37.00 & 0.00 \\
\hline B44 & Ft. Benning & $\begin{array}{c}2852 . \\
70\end{array}$ & $\begin{array}{c}2524 . \\
00\end{array}$ & $\begin{array}{c}25960 \\
5.60\end{array}$ & $\begin{array}{c}167 . \\
60\end{array}$ & $\begin{array}{c}153.0 \\
0\end{array}$ & 0.00 & 53.60 & - & $\begin{array}{c}2149 \\
.35\end{array}$ & 25.10 & 39.00 & 0.00 \\
\hline B45 & Ft. Benning & $\begin{array}{c}2494 . \\
90\end{array}$ & $\begin{array}{c}1842 . \\
00\end{array}$ & $\begin{array}{c}52591 \\
.67\end{array}$ & $\begin{array}{c}132 . \\
70\end{array}$ & - & 0.00 & 31.30 & - & $\begin{array}{c}386 . \\
26\end{array}$ & 33.40 & 30.00 & 0.00 \\
\hline B54 & Ft. Benning & $\begin{array}{c}8360 . \\
90\end{array}$ & $\begin{array}{c}6210 . \\
00\end{array}$ & $\begin{array}{c}30527 \\
6.90\end{array}$ & $\begin{array}{c}135 . \\
60\end{array}$ & - & 0.00 & $\begin{array}{c}145.0 \\
0\end{array}$ & $\begin{array}{c}100.0 \\
0\end{array}$ & $\begin{array}{c}2374 \\
.32\end{array}$ & 56.60 & 68.00 & 0.00 \\
\hline B55 & Ft. Benning & $\begin{array}{l}1140 \\
0.10\end{array}$ & $\begin{array}{c}5720 . \\
00\end{array}$ & $\begin{array}{c}31724 \\
4.40\end{array}$ & $\begin{array}{c}132 . \\
70\end{array}$ & - & 0.00 & $\begin{array}{c}191.8 \\
0\end{array}$ & 73.00 & $\begin{array}{c}973 . \\
96\end{array}$ & $\begin{array}{c}263.3 \\
0\end{array}$ & 63.00 & 0.00 \\
\hline B56 & Ft. Benning & $\begin{array}{c}5292 . \\
20\end{array}$ & $\begin{array}{c}6618 . \\
00 \\
\end{array}$ & $\begin{array}{c}23661 \\
9.40 \\
\end{array}$ & $\begin{array}{c}69.3 \\
0 \\
\end{array}$ & - & 0.00 & $\begin{array}{c}137.6 \\
0\end{array}$ & $\begin{array}{c}118.0 \\
0\end{array}$ & $\begin{array}{c}680 . \\
11\end{array}$ & 56.40 & 76.00 & 0.00 \\
\hline B57 & Ft. Benning & $\begin{array}{c}8765 . \\
10 \\
\end{array}$ & $\begin{array}{c}8011 . \\
00\end{array}$ & $\begin{array}{c}44212 \\
.04 \\
\end{array}$ & $\begin{array}{c}18.4 \\
0 \\
\end{array}$ & - & 0.00 & 66.40 & 98.00 & $\begin{array}{c}335 . \\
76 \\
\end{array}$ & 69.80 & 97.00 & $\begin{array}{c}1910 . \\
20 \\
\end{array}$ \\
\hline B58 & Ft. Benning & $\begin{array}{c}9981 . \\
00\end{array}$ & $\begin{array}{c}8155 . \\
00\end{array}$ & $\begin{array}{c}31655 \\
0.00\end{array}$ & $\begin{array}{c}133 . \\
00\end{array}$ & - & 0.00 & $\begin{array}{c}146.4 \\
0\end{array}$ & $\begin{array}{c}117.0 \\
0\end{array}$ & $\begin{array}{c}46.5 \\
0\end{array}$ & $\begin{array}{c}100.0 \\
0\end{array}$ & 97.00 & 0.00 \\
\hline B59 & Ft. Benning & $\begin{array}{l}1038 \\
5.30\end{array}$ & $\begin{array}{c}6409 . \\
00\end{array}$ & $\begin{array}{c}30900 \\
3.70\end{array}$ & $\begin{array}{c}214 . \\
30\end{array}$ & $\begin{array}{c}208.0 \\
0\end{array}$ & 0.00 & $\begin{array}{c}188.3 \\
0\end{array}$ & 96.00 & $\begin{array}{c}1612 \\
.16\end{array}$ & 61.70 & 76.00 & $\begin{array}{c}190.2 \\
6\end{array}$ \\
\hline B60 & Ft. Benning & $\begin{array}{c}4801 . \\
50\end{array}$ & $\begin{array}{c}2823 . \\
00\end{array}$ & $\begin{array}{c}64142 \\
.59 \\
\end{array}$ & $\begin{array}{c}38.3 \\
0\end{array}$ & - & 0.00 & 56.40 & - & $\begin{array}{c}670 . \\
93\end{array}$ & 31.60 & 45.00 & 0.00 \\
\hline B61 & Ft. Benning & $\begin{array}{c}2942 . \\
80 \\
\end{array}$ & $\begin{array}{c}3432 . \\
00\end{array}$ & $\begin{array}{c}67545 \\
.37 \\
\end{array}$ & $\begin{array}{c}118 . \\
40 \\
\end{array}$ & $\begin{array}{c}126.0 \\
0 \\
\end{array}$ & 0.00 & 46.10 & - & $\begin{array}{c}1580 \\
.02 \\
\end{array}$ & 24.00 & 39.00 & 0.00 \\
\hline B62 & Ft. Benning & $\begin{array}{c}8300 . \\
30\end{array}$ & $\begin{array}{c}3320 . \\
00\end{array}$ & $\begin{array}{c}56341 \\
.67\end{array}$ & $\begin{array}{c}131 . \\
40\end{array}$ & - & 0.00 & $\begin{array}{c}142.0 \\
0\end{array}$ & - & $\begin{array}{c}1130 \\
.06\end{array}$ & $\begin{array}{c}131.2 \\
0\end{array}$ & 58.00 & 0.00 \\
\hline B63 & Ft. Benning & $\begin{array}{c}3600 . \\
10 \\
\end{array}$ & $\begin{array}{c}3395 . \\
00 \\
\end{array}$ & $\begin{array}{c}16828 \\
6.10 \\
\end{array}$ & $\begin{array}{c}56.3 \\
0 \\
\end{array}$ & - & 0.00 & 54.30 & - & $\begin{array}{c}225 . \\
56\end{array}$ & 38.70 & 52.00 & $\begin{array}{c}182.6 \\
5 \\
\end{array}$ \\
\hline B64 & Ft. Benning & $\begin{array}{c}2938 . \\
80 \\
\end{array}$ & $\begin{array}{c}1652 . \\
00\end{array}$ & $\begin{array}{c}12198 \\
.15 \\
\end{array}$ & $\begin{array}{c}91.5 \\
0 \\
\end{array}$ & - & 0.00 & 47.30 & - & 0.00 & 25.90 & 38.00 & 0.00 \\
\hline B65 & Ft. Benning & $\begin{array}{c}2790 . \\
50\end{array}$ & $\begin{array}{c}1314 . \\
00\end{array}$ & $\begin{array}{c}1480 . \\
56\end{array}$ & $\begin{array}{c}15.9 \\
0\end{array}$ & - & 0.00 & 15.70 & - & $\begin{array}{c}638 . \\
79\end{array}$ & 32.00 & 30.00 & 0.00 \\
\hline
\end{tabular}




\begin{tabular}{|c|c|c|c|c|c|c|c|c|c|c|c|c|c|}
\hline B66 & Ft. Benning & $\begin{array}{l}1043 \\
3.20\end{array}$ & $\begin{array}{c}1798 . \\
00\end{array}$ & $\begin{array}{c}27383 \\
.33\end{array}$ & $\begin{array}{c}28.4 \\
0\end{array}$ & - & 0.00 & $\begin{array}{c}281.0 \\
0\end{array}$ & - & $\begin{array}{c}565 \\
33\end{array}$ & $\begin{array}{c}949.2 \\
0\end{array}$ & 45.00 & 0.00 \\
\hline B70 & Ft. Benning & $\begin{array}{c}5234 . \\
30\end{array}$ & $\begin{array}{c}4987 . \\
00\end{array}$ & $\begin{array}{c}16307 \\
7.80\end{array}$ & $\begin{array}{c}91.5 \\
0\end{array}$ & - & 0.00 & 78.40 & - & $\begin{array}{c}1598 \\
.38\end{array}$ & 48.50 & 66.00 & 0.00 \\
\hline B71 & Ft. Benning & $\begin{array}{c}5516 . \\
00\end{array}$ & $\begin{array}{c}5528 . \\
00\end{array}$ & $\begin{array}{c}19062 \\
4.10\end{array}$ & $\begin{array}{c}98.7 \\
0 \\
\end{array}$ & - & 0.00 & 70.80 & 62.00 & $\begin{array}{c}748 . \\
98\end{array}$ & 46.80 & 72.00 & 0.00 \\
\hline B74 & Ft. Benning & $\begin{array}{c}329.9 \\
0\end{array}$ & $\begin{array}{c}526.0 \\
0\end{array}$ & 0.00 & - & - & 0.00 & - & - & $\begin{array}{c}299 . \\
03\end{array}$ & 17.90 & 37.00 & 0.00 \\
\hline B75 & Ft. Benning & $\begin{array}{c}314.7 \\
0\end{array}$ & $\begin{array}{c}689.0 \\
0 \\
\end{array}$ & 0.00 & - & - & 0.00 & - & - & $\begin{array}{c}459 . \\
72\end{array}$ & 19.70 & 39.00 & 0.00 \\
\hline B76 & Ft. Benning & $\begin{array}{c}3141 . \\
80\end{array}$ & $\begin{array}{c}3476 . \\
00 \\
\end{array}$ & $\begin{array}{c}59813 \\
.89 \\
\end{array}$ & $\begin{array}{c}83.8 \\
0 \\
\end{array}$ & - & 0.00 & 34.50 & - & $\begin{array}{c}2048 \\
.34 \\
\end{array}$ & 30.80 & 56.00 & 0.00 \\
\hline B77 & Ft. Benning & $\begin{array}{c}5748 . \\
40\end{array}$ & $\begin{array}{c}3601 . \\
00\end{array}$ & $\begin{array}{c}20437 \\
4.10\end{array}$ & $\begin{array}{c}65.5 \\
0\end{array}$ & - & 0.00 & 70.30 & - & $\begin{array}{c}1942 \\
.74\end{array}$ & 54.00 & 58.00 & 0.00 \\
\hline B78 & Ft. Benning & $\begin{array}{c}1039 \\
10\end{array}$ & $\begin{array}{c}947.0 \\
0\end{array}$ & $\begin{array}{c}36897 \\
.22\end{array}$ & $\begin{array}{c}13.8 \\
0\end{array}$ & - & 0.00 & 14.60 & - & $\begin{array}{c}813 . \\
26\end{array}$ & 11.60 & 22.00 & 0.00 \\
\hline B79 & Ft. Benning & $\begin{array}{c}613.3 \\
0\end{array}$ & $\begin{array}{c}1081 . \\
00\end{array}$ & 0.00 & $\begin{array}{c}40.0 \\
0\end{array}$ & - & 0.00 & 9.50 & - & $\begin{array}{c}1997 \\
.83\end{array}$ & 9.50 & 27.00 & 0.00 \\
\hline $\mathrm{B} 80$ & Ft. Benning & $\begin{array}{c}202.7 \\
0\end{array}$ & $\begin{array}{c}368.0 \\
0\end{array}$ & 0.00 & - & - & 0.00 & - & - & $\begin{array}{c}1134 \\
.66\end{array}$ & 71.20 & 12.00 & 0.00 \\
\hline B81 & Ft. Benning & $\begin{array}{c}3564 . \\
50\end{array}$ & $\begin{array}{c}5606 . \\
00\end{array}$ & $\begin{array}{c}10583 \\
2.40\end{array}$ & $\begin{array}{c}20.2 \\
0 \\
\end{array}$ & - & 0.00 & 48.60 & 83.00 & $\begin{array}{c}1906 \\
.01\end{array}$ & 30.40 & 73.00 & 0.00 \\
\hline B82 & Ft. Benning & $\begin{array}{c}1104 . \\
90\end{array}$ & $\begin{array}{c}1687 . \\
00\end{array}$ & $\begin{array}{c}35137 \\
.96\end{array}$ & - & - & 0.00 & 8.00 & - & $\begin{array}{c}960 . \\
18 \\
\end{array}$ & 12.90 & 38.00 & 0.00 \\
\hline $\mathrm{B} 83$ & Ft. Benning & 85.60 & $\begin{array}{c}126.0 \\
0 \\
\end{array}$ & 0.00 & - & - & 0.00 & - & - & $\begin{array}{c}468 . \\
91 \\
\end{array}$ & 9.60 & 18.00 & 0.00 \\
\hline B84 & Ft. Benning & 51.80 & 92.00 & 0.00 & 2.40 & - & 0.00 & 4.20 & - & $\begin{array}{c}491 . \\
86 \\
\end{array}$ & 8.40 & 32.00 & 0.00 \\
\hline B85 & Ft. Benning & 96.00 & $\begin{array}{c}116.0 \\
0\end{array}$ & 0.00 & 3.70 & - & 0.00 & 9.20 & - & $\begin{array}{c}2553 \\
.39\end{array}$ & 14.80 & 27.00 & $\begin{array}{c}1621 . \\
01\end{array}$ \\
\hline B86 & Ft. Benning & 81.50 & $\begin{array}{c}106.0 \\
0 \\
\end{array}$ & 0.00 & 4.20 & - & 0.00 & 7.40 & - & $\begin{array}{c}2911 \\
.51 \\
\end{array}$ & 12.10 & 27.00 & $\begin{array}{c}570.7 \\
8\end{array}$ \\
\hline B87 & Ft. Benning & $\begin{array}{c}1217 . \\
60 \\
\end{array}$ & $\begin{array}{c}907.0 \\
0 \\
\end{array}$ & $\begin{array}{c}11758 \\
.33 \\
\end{array}$ & $\begin{array}{c}102 . \\
70 \\
\end{array}$ & $\begin{array}{c}175.0 \\
0 \\
\end{array}$ & 0.00 & 15.00 & - & $\begin{array}{c}400 . \\
04 \\
\end{array}$ & 21.10 & 29.00 & 0.00 \\
\hline $\mathrm{B} 88$ & Ft. Benning & $\begin{array}{c}102.7 \\
0\end{array}$ & $\begin{array}{c}106.0 \\
0\end{array}$ & 0.00 & - & - & 0.00 & 6.50 & - & $\begin{array}{c}2980 \\
.39 \\
\end{array}$ & 15.40 & 22.00 & $\begin{array}{c}1118 . \\
72\end{array}$ \\
\hline B89 & Ft. Benning & 51.00 & 52.00 & 0.00 & $\begin{array}{c}14.9 \\
0\end{array}$ & - & 0.00 & 14.90 & - & $\begin{array}{c}3499 \\
.21\end{array}$ & 19.60 & 29.00 & $\begin{array}{c}418.5 \\
7\end{array}$ \\
\hline B90 & Ft. Benning & 43.80 & 42.00 & 0.00 & $\begin{array}{c}24.3 \\
0 \\
\end{array}$ & - & 0.00 & 19.50 & - & $\begin{array}{c}4729 \\
.70 \\
\end{array}$ & 35.30 & 37.00 & 0.00 \\
\hline B91 & Ft. Benning & 41.90 & 47.00 & $\begin{array}{c}45577 \\
.78\end{array}$ & $\begin{array}{c}42.4 \\
0\end{array}$ & - & 0.00 & 18.20 & - & $\begin{array}{c}703 . \\
07\end{array}$ & 13.10 & 29.00 & $\begin{array}{c}1537 . \\
29\end{array}$ \\
\hline B92 & Ft. Benning & 71.30 & 77.00 & 0.00 & $\begin{array}{c}40.1 \\
0\end{array}$ & - & 0.00 & 23.50 & - & $\begin{array}{c}4109 \\
.86\end{array}$ & 17.40 & 32.00 & $\begin{array}{c}410.9 \\
6\end{array}$ \\
\hline B95 & Ft. Benning & 9.60 & 81.00 & 0.00 & - & - & 0.00 & - & - & $\begin{array}{c}253 . \\
11 \\
\end{array}$ & 30.50 & $\begin{array}{c}134.0 \\
0 \\
\end{array}$ & 0.00 \\
\hline B96 & Ft. Benning & 12.00 & 77.00 & 0.00 & - & - & 0.00 & - & - & 0.00 & 24.50 & $\begin{array}{c}178.0 \\
0 \\
\end{array}$ & $\begin{array}{c}669.7 \\
1 \\
\end{array}$ \\
\hline FE D 01 & Ft. Eustis & $\begin{array}{c}235.1 \\
0\end{array}$ & $\begin{array}{c}581.0 \\
0\end{array}$ & 0.00 & - & - & 0.00 & - & - & $\begin{array}{c}1428 \\
.50 \\
\end{array}$ & 18.90 & 41.00 & 0.00 \\
\hline FE D 02 & Ft. Eustis & $\begin{array}{c}2571 . \\
50\end{array}$ & $\begin{array}{c}1572 . \\
00\end{array}$ & 0.00 & - & - & 0.00 & $\begin{array}{c}396.3 \\
0\end{array}$ & - & 0.00 & 20.90 & 61.00 & $\begin{array}{c}190.2 \\
6\end{array}$ \\
\hline FE D 03 & Ft. Eustis & $\begin{array}{c}158.9 \\
0\end{array}$ & $\begin{array}{c}358.0 \\
0\end{array}$ & 0.00 & - & - & 0.00 & - & - & $\begin{array}{c}1791 \\
.22 \\
\end{array}$ & 16.60 & 40.00 & 0.00 \\
\hline FE D 04 & Ft. Eustis & $\begin{array}{c}156.5 \\
0 \\
\end{array}$ & $\begin{array}{c}365.0 \\
0\end{array}$ & 0.00 & - & - & 0.00 & - & - & $\begin{array}{c}592 . \\
87\end{array}$ & 20.30 & 52.00 & 0.00 \\
\hline FE D 05 & Ft. Eustis & $\begin{array}{c}199.0 \\
0\end{array}$ & $\begin{array}{c}480.0 \\
0\end{array}$ & 0.00 & - & - & 0.00 & - & - & $\begin{array}{c}1896 \\
.82\end{array}$ & 19.20 & 51.00 & 0.00 \\
\hline
\end{tabular}




\begin{tabular}{|c|c|c|c|c|c|c|c|c|c|c|c|c|c|}
\hline FE D 06 & Ft. Eustis & $\begin{array}{c}197.9 \\
0\end{array}$ & $\begin{array}{c}616.0 \\
0\end{array}$ & 0.00 & - & - & 0.00 & - & - & $\begin{array}{c}712 . \\
25\end{array}$ & 20.90 & 58.00 & 0.00 \\
\hline FE D 07 & Ft. Eustis & 47.30 & $\begin{array}{c}181.0 \\
0\end{array}$ & 0.00 & - & - & 0.00 & - & - & $\begin{array}{c}1515 \\
.74\end{array}$ & 8.50 & 31.00 & 0.00 \\
\hline FE D 08 & Ft. Eustis & 55.60 & $\begin{array}{c}169.0 \\
0 \\
\end{array}$ & 0.00 & - & - & 0.00 & - & - & $\begin{array}{c}1777 \\
.45 \\
\end{array}$ & 10.30 & 38.00 & 0.00 \\
\hline FE D 09 & Ft. Eustis & 17.90 & 78.00 & 0.00 & - & - & 0.00 & - & - & 0.00 & 21.30 & 82.00 & $\begin{array}{c}106.5 \\
4\end{array}$ \\
\hline FE D 10 & Ft. Eustis & 3.00 & 38.00 & 0.00 & - & - & 0.00 & - & - & $\begin{array}{c}2374 \\
.32\end{array}$ & 19.50 & 58.00 & 0.00 \\
\hline FE D 11 & Ft. Eustis & 40.40 & $\begin{array}{c}103.0 \\
0\end{array}$ & 0.00 & - & - & 0.00 & - & - & $\begin{array}{c}1391 \\
.77 \\
\end{array}$ & 20.10 & 50.00 & $\begin{array}{c}593.6 \\
1 \\
\end{array}$ \\
\hline FE D 12 & Ft. Eustis & $\begin{array}{c}2955 . \\
90\end{array}$ & $\begin{array}{c}2626 . \\
00\end{array}$ & 0.00 & - & - & 0.00 & - & - & $\begin{array}{c}1410 \\
.14\end{array}$ & 22.70 & 50.00 & 15.22 \\
\hline FE D 13 & Ft. Eustis & $\begin{array}{c}176.2 \\
0\end{array}$ & $\begin{array}{c}537.0 \\
0\end{array}$ & 0.00 & - & - & 0.00 & - & - & $\begin{array}{c}1272 \\
.40\end{array}$ & 16.10 & 41.00 & 0.00 \\
\hline FE D 14 & Ft. Eustis & $\begin{array}{c}157.9 \\
0\end{array}$ & $\begin{array}{c}347.0 \\
0\end{array}$ & 0.00 & - & - & 0.00 & - & - & $\begin{array}{c}951 . \\
00\end{array}$ & 14.70 & 34.00 & 0.00 \\
\hline FE D 16 & Ft. Eustis & $\begin{array}{c}235.1 \\
0\end{array}$ & $\begin{array}{c}754.0 \\
0\end{array}$ & 0.00 & - & - & 0.00 & - & - & $\begin{array}{c}1189 \\
.75\end{array}$ & 15.20 & 48.00 & 0.00 \\
\hline FE D 17 & Ft. Eustis & $\begin{array}{c}352.9 \\
0 \\
\end{array}$ & $\begin{array}{c}940.0 \\
0 \\
\end{array}$ & 0.00 & - & - & 0.00 & - & - & $\begin{array}{c}1249 \\
.44 \\
\end{array}$ & 15.10 & 47.00 & 0.00 \\
\hline FE D 18 & Ft. Eustis & 63.60 & $\begin{array}{c}187.0 \\
0\end{array}$ & 0.00 & - & - & 0.00 & - & - & $\begin{array}{c}546 . \\
96\end{array}$ & 14.80 & 48.00 & 0.00 \\
\hline FE D 19 & Ft. Eustis & 29.00 & $\begin{array}{c}100.0 \\
0\end{array}$ & 0.00 & - & - & 0.00 & - & - & $\begin{array}{c}859 . \\
17\end{array}$ & 15.90 & 51.00 & 0.00 \\
\hline FE D 20 & Ft. Eustis & 31.90 & 89.00 & 0.00 & - & - & 0.00 & - & - & $\begin{array}{c}951 . \\
00\end{array}$ & 16.50 & 51.00 & 0.00 \\
\hline FE D 21 & Ft. Eustis & 8.70 & 41.00 & 0.00 & - & - & 0.00 & - & - & $\begin{array}{c}1887 \\
.64\end{array}$ & 18.30 & 54.00 & 0.00 \\
\hline FE D 22 & Ft. Eustis & 40.70 & $\begin{array}{c}102.0 \\
0\end{array}$ & 0.00 & - & - & 0.00 & - & - & $\begin{array}{c}758 . \\
16\end{array}$ & 23.40 & 56.00 & 30.44 \\
\hline FE D 23 & Ft. Eustis & 63.10 & $\begin{array}{c}207.0 \\
0\end{array}$ & 0.00 & - & - & 0.00 & - & - & $\begin{array}{c}335 . \\
76\end{array}$ & 31.40 & 81.00 & 0.00 \\
\hline FE D 24 & Ft. Eustis & 39.30 & $\begin{array}{c}138.0 \\
0 \\
\end{array}$ & 0.00 & - & - & 0.00 & - & - & $\begin{array}{c}946 . \\
41 \\
\end{array}$ & 22.30 & $\begin{array}{c}101.0 \\
0\end{array}$ & 0.00 \\
\hline FE D 25 & Ft. Eustis & 33.70 & 78.00 & 0.00 & - & - & 0.00 & - & - & $\begin{array}{c}1483 \\
.60\end{array}$ & 14.30 & 43.00 & $\begin{array}{c}258.7 \\
5\end{array}$ \\
\hline FE D 26 & Ft. Eustis & 28.50 & 80.00 & 0.00 & - & - & 0.00 & - & - & $\begin{array}{c}156 . \\
69\end{array}$ & 14.40 & 30.00 & 0.00 \\
\hline FE D 27 & Ft. Eustis & 17.10 & 83.00 & 0.00 & - & - & 0.00 & - & - & 0.00 & 11.70 & 55.00 & 0.00 \\
\hline FE D 28 & Ft. Eustis & 23.70 & 82.00 & 0.00 & - & - & 0.00 & - & - & $\begin{array}{c}804 . \\
08\end{array}$ & 12.10 & 42.00 & 0.00 \\
\hline FE D 29 & Ft. Eustis & 10.90 & 52.00 & 0.00 & - & - & 0.00 & - & - & $\begin{array}{c}849 . \\
99\end{array}$ & 11.00 & 38.00 & 0.00 \\
\hline FE D 30 & Ft. Eustis & - & 35.00 & 0.00 & - & - & 0.00 & - & - & $\begin{array}{c}1387 \\
.18\end{array}$ & 11.20 & 45.00 & $\begin{array}{c}273.9 \\
7\end{array}$ \\
\hline FE D 31 & Ft. Eustis & 20.50 & $\begin{array}{c}122.0 \\
0\end{array}$ & 0.00 & - & - & 0.00 & - & - & $\begin{array}{c}1212 \\
.71\end{array}$ & 9.70 & 50.00 & 0.00 \\
\hline FE D 32 & Ft. Eustis & - & 32.00 & 0.00 & - & - & 0.00 & - & - & $\begin{array}{c}1855 \\
.50 \\
\end{array}$ & 12.90 & 47.00 & 0.00 \\
\hline FE D 33 & Ft. Eustis & - & 33.00 & 0.00 & - & - & 0.00 & - & - & $\begin{array}{c}1111 \\
.70\end{array}$ & 10.90 & 50.00 & 0.00 \\
\hline $\begin{array}{c}\text { FE MI- } \\
01\end{array}$ & Ft. Eustis & $\begin{array}{c}183.0 \\
0\end{array}$ & $\begin{array}{c}482.0 \\
0\end{array}$ & $\begin{array}{c}22059 \\
.26\end{array}$ & - & - & 0.00 & - & - & $\begin{array}{c}1728 \\
.09\end{array}$ & 14.40 & 41.00 & 0.00 \\
\hline $\begin{array}{c}\text { FE MI- } \\
02\end{array}$ & Ft. Eustis & $\begin{array}{c}232.5 \\
0\end{array}$ & $\begin{array}{c}571.0 \\
0\end{array}$ & 0.00 & - & - & 0.00 & - & - & $\begin{array}{c}1429 \\
.65\end{array}$ & 14.20 & 45.00 & 0.00 \\
\hline
\end{tabular}




\begin{tabular}{|c|c|c|c|c|c|c|c|c|c|c|c|c|c|}
\hline $\begin{array}{c}\text { FE MI- } \\
03\end{array}$ & Ft. Eustis & $\begin{array}{c}237.9 \\
0\end{array}$ & $\begin{array}{c}568.0 \\
0\end{array}$ & 0.00 & - & - & 0.00 & - & - & $\begin{array}{c}1089 \\
.89\end{array}$ & 15.10 & 42.00 & 0.00 \\
\hline $\begin{array}{c}\text { FE MI- } \\
04\end{array}$ & Ft. Eustis & $\begin{array}{c}357.5 \\
0\end{array}$ & $\begin{array}{c}860.0 \\
0\end{array}$ & 0.00 & - & - & 0.00 & - & - & $\begin{array}{c}162 . \\
43\end{array}$ & 17.00 & 52.00 & 0.00 \\
\hline $\begin{array}{c}\text { FE MI- } \\
05\end{array}$ & Ft. Eustis & $\begin{array}{c}713.8 \\
0\end{array}$ & $\begin{array}{c}1389 . \\
00\end{array}$ & 0.00 & - & - & 0.00 & 7.80 & - & $\begin{array}{c}534 . \\
33\end{array}$ & 18.30 & 46.00 & 22.83 \\
\hline $\begin{array}{c}\text { FE MI- } \\
06\end{array}$ & Ft. Eustis & $\begin{array}{c}360.5 \\
0\end{array}$ & $\begin{array}{c}762.0 \\
0\end{array}$ & $\begin{array}{c}3355 . \\
56\end{array}$ & - & - & 0.00 & - & - & $\begin{array}{c}75.2 \\
0\end{array}$ & 17.00 & 43.00 & 0.00 \\
\hline $\begin{array}{c}\text { FE MI- } \\
07 \\
\end{array}$ & Ft. Eustis & $\begin{array}{c}475.8 \\
0 \\
\end{array}$ & $\begin{array}{c}1121 . \\
00\end{array}$ & $\begin{array}{c}7776 . \\
85\end{array}$ & - & - & 0.00 & 3.30 & - & $\begin{array}{c}1369 \\
.96 \\
\end{array}$ & 17.50 & 38.00 & $\begin{array}{c}441.4 \\
0\end{array}$ \\
\hline $\begin{array}{c}\text { FE MI- } \\
08 \\
\end{array}$ & Ft. Eustis & $\begin{array}{c}643.0 \\
0 \\
\end{array}$ & $\begin{array}{c}1288 . \\
00\end{array}$ & 0.00 & - & - & 0.00 & 3.40 & - & $\begin{array}{c}1227 \\
.63 \\
\end{array}$ & 16.50 & 46.00 & $\begin{array}{c}144.6 \\
0 \\
\end{array}$ \\
\hline $\begin{array}{c}\text { FE MI- } \\
09\end{array}$ & Ft. Eustis & $\begin{array}{c}432.0 \\
0\end{array}$ & $\begin{array}{c}986.0 \\
0\end{array}$ & 0.00 & - & - & 0.00 & - & - & $\begin{array}{c}1062 \\
.34\end{array}$ & 15.50 & 43.00 & 0.00 \\
\hline $\begin{array}{c}\text { FE MI- } \\
10\end{array}$ & Ft. Eustis & $\begin{array}{c}388.2 \\
0\end{array}$ & $\begin{array}{c}770.0 \\
0\end{array}$ & $\begin{array}{c}9698 . \\
15\end{array}$ & - & - & 0.00 & 4.20 & - & $\begin{array}{c}323 . \\
13\end{array}$ & 16.40 & 40.00 & $\begin{array}{c}220.7 \\
0\end{array}$ \\
\hline $\begin{array}{c}\text { FE MI- } \\
11\end{array}$ & Ft. Eustis & $\begin{array}{c}382.2 \\
0\end{array}$ & $\begin{array}{c}671.0 \\
0\end{array}$ & 0.00 & - & - & 0.00 & - & - & $\begin{array}{c}217 . \\
53\end{array}$ & 17.40 & 42.00 & $\begin{array}{c}2640 . \\
79\end{array}$ \\
\hline $\begin{array}{c}\text { FE MI- } \\
12\end{array}$ & Ft. Eustis & $\begin{array}{c}244.8 \\
0\end{array}$ & $\begin{array}{c}572.0 \\
0\end{array}$ & 0.00 & - & - & 0.00 & - & - & $\begin{array}{c}144 . \\
07\end{array}$ & 17.50 & 44.00 & $\begin{array}{c}1270 . \\
93\end{array}$ \\
\hline $\begin{array}{c}\text { FE MI- } \\
13\end{array}$ & Ft. Eustis & $\begin{array}{c}564.5 \\
0\end{array}$ & $\begin{array}{c}1041 . \\
00\end{array}$ & 0.00 & - & - & 0.00 & 2.40 & - & $\begin{array}{c}355 . \\
27\end{array}$ & 17.50 & 45.00 & 0.00 \\
\hline $\begin{array}{c}\text { FE MI- } \\
14 \\
\end{array}$ & Ft. Eustis & $\begin{array}{c}562.5 \\
0\end{array}$ & $\begin{array}{c}1123 . \\
00\end{array}$ & 0.00 & - & - & 0.00 & 7.90 & - & $\begin{array}{c}102 . \\
75\end{array}$ & 16.90 & 39.00 & $\begin{array}{c}449.0 \\
1 \\
\end{array}$ \\
\hline $\begin{array}{c}\text { FE MI- } \\
15 \\
\end{array}$ & Ft. Eustis & $\begin{array}{c}501.9 \\
0 \\
\end{array}$ & $\begin{array}{c}996.0 \\
0 \\
\end{array}$ & 0.00 & - & - & 0.00 & - & - & $\begin{array}{c}84.3 \\
8 \\
\end{array}$ & 16.60 & 45.00 & 0.00 \\
\hline $\begin{array}{c}\text { FE MI- } \\
16\end{array}$ & Ft. Eustis & $\begin{array}{c}565.2 \\
0\end{array}$ & $\begin{array}{c}1253 . \\
00\end{array}$ & 0.00 & - & - & 0.00 & 2.40 & - & $\begin{array}{c}240 . \\
49\end{array}$ & 20.70 & 46.00 & 0.00 \\
\hline $\begin{array}{c}\text { FE MI- } \\
17\end{array}$ & Ft. Eustis & $\begin{array}{c}822.5 \\
0\end{array}$ & $\begin{array}{c}1649 . \\
00\end{array}$ & 0.00 & - & - & 0.00 & 7.00 & - & $\begin{array}{c}672 . \\
08\end{array}$ & 21.00 & 45.00 & 0.00 \\
\hline $\begin{array}{c}\text { FE MI- } \\
18\end{array}$ & Ft. Eustis & $\begin{array}{c}501.1 \\
0\end{array}$ & $\begin{array}{c}990.0 \\
0\end{array}$ & 0.00 & - & - & 0.00 & - & - & $\begin{array}{c}621 . \\
57\end{array}$ & 18.10 & 38.00 & 0.00 \\
\hline $\begin{array}{c}\text { FE MI- } \\
19\end{array}$ & Ft. Eustis & 75.00 & $\begin{array}{c}213.0 \\
0\end{array}$ & 0.00 & - & - & 0.00 & - & - & $\begin{array}{c}727 . \\
17\end{array}$ & 14.10 & 37.00 & 0.00 \\
\hline $\begin{array}{c}\text { FE MI- } \\
20\end{array}$ & Ft. Eustis & $\begin{array}{c}118.2 \\
0\end{array}$ & $\begin{array}{c}321.0 \\
0\end{array}$ & 0.00 & - & - & 0.00 & - & - & $\begin{array}{c}1291 \\
.91\end{array}$ & 13.20 & 39.00 & $\begin{array}{c}258.7 \\
5\end{array}$ \\
\hline $\begin{array}{c}\text { FE MI- } \\
21\end{array}$ & Ft. Eustis & 76.50 & $\begin{array}{c}222.0 \\
0\end{array}$ & 0.00 & - & - & 0.00 & - & - & $\begin{array}{c}1539 \\
.84 \\
\end{array}$ & 13.40 & 38.00 & $\begin{array}{c}395.7 \\
4 \\
\end{array}$ \\
\hline $\begin{array}{c}\text { FE MI- } \\
22 \\
\end{array}$ & Ft. Eustis & $\begin{array}{c}620.3 \\
0\end{array}$ & $\begin{array}{c}1245 . \\
00\end{array}$ & $\begin{array}{c}14883 \\
.33 \\
\end{array}$ & - & - & 0.00 & - & - & $\begin{array}{c}695 . \\
03\end{array}$ & 16.10 & 45.00 & 0.00 \\
\hline $\begin{array}{c}\text { FE MI- } \\
23 \\
\end{array}$ & Ft. Eustis & $\begin{array}{c}588.4 \\
0 \\
\end{array}$ & $\begin{array}{c}1083 . \\
00\end{array}$ & 0.00 & 4.80 & - & 0.00 & 20.70 & - & $\begin{array}{c}561 . \\
88 \\
\end{array}$ & 17.40 & 47.00 & $\begin{array}{c}1035 . \\
01\end{array}$ \\
\hline $\begin{array}{c}\text { FE MI- } \\
24\end{array}$ & Ft. Eustis & $\begin{array}{c}623.5 \\
0\end{array}$ & $\begin{array}{c}1199 . \\
00\end{array}$ & 0.00 & - & - & 0.00 & - & - & $\begin{array}{c}414 . \\
96\end{array}$ & 15.30 & 45.00 & $\begin{array}{c}707.7 \\
6\end{array}$ \\
\hline $\begin{array}{c}\text { FE MI- } \\
25 \\
\end{array}$ & Ft. Eustis & $\begin{array}{c}599.5 \\
0 \\
\end{array}$ & $\begin{array}{c}1112 . \\
00\end{array}$ & 0.00 & - & - & 0.00 & - & - & 0.00 & 17.20 & 44.00 & $\begin{array}{c}601.2 \\
2 \\
\end{array}$ \\
\hline $\begin{array}{c}\text { FE MI- } \\
26\end{array}$ & Ft. Eustis & $\begin{array}{c}478.3 \\
0\end{array}$ & $\begin{array}{c}944.0 \\
0\end{array}$ & 0.00 & - & - & 0.00 & - & - & $\begin{array}{c}451 . \\
69\end{array}$ & 16.30 & 36.00 & 0.00 \\
\hline $\begin{array}{c}\text { FE MI- } \\
27\end{array}$ & Ft. Eustis & $\begin{array}{c}672.4 \\
0\end{array}$ & $\begin{array}{c}1145 . \\
00\end{array}$ & $\begin{array}{c}7128 . \\
70\end{array}$ & - & - & 0.00 & 3.20 & - & $\begin{array}{c}759 . \\
31\end{array}$ & 16.60 & 36.00 & 0.00 \\
\hline FL 01 & Ft. Lewis & 30.70 & $\begin{array}{c}107.0 \\
0\end{array}$ & 0.00 & - & - & 0.00 & - & - & $\begin{array}{c}1244 \\
.85\end{array}$ & 19.60 & 56.00 & 0.00 \\
\hline FL 02 & Ft. Lewis & 28.80 & 95.00 & 0.00 & - & - & 0.00 & - & - & $\begin{array}{c}1915 \\
.19\end{array}$ & 19.10 & 54.00 & $\begin{array}{c}388.1 \\
3 \\
\end{array}$ \\
\hline FL 03 & Ft. Lewis & 78.60 & $\begin{array}{c}279.0 \\
0\end{array}$ & 0.00 & - & - & 0.00 & - & - & $\begin{array}{c}1217 \\
.30\end{array}$ & 17.20 & $\begin{array}{c}153.0 \\
0\end{array}$ & 0.00 \\
\hline FL 04 & Ft. Lewis & $\begin{array}{c}130.4 \\
0\end{array}$ & $\begin{array}{c}194.0 \\
0\end{array}$ & 0.00 & - & - & 0.00 & - & - & $\begin{array}{c}2310 \\
.05\end{array}$ & 18.40 & 47.00 & $\begin{array}{c}175.0 \\
4\end{array}$ \\
\hline
\end{tabular}




\begin{tabular}{|c|c|c|c|c|c|c|c|c|c|c|c|c|c|}
\hline FL 05 & Ft. Lewis & $\begin{array}{c}7539 . \\
50\end{array}$ & $\begin{array}{l}1096 \\
3.00\end{array}$ & $\begin{array}{c}10379 \\
5.40\end{array}$ & $\begin{array}{c}130 . \\
30\end{array}$ & - & 0.00 & $\begin{array}{c}245.0 \\
0\end{array}$ & $\begin{array}{c}219.0 \\
0\end{array}$ & $\begin{array}{c}2153 \\
.94\end{array}$ & $\begin{array}{c}133.4 \\
0\end{array}$ & $\begin{array}{c}171.0 \\
0\end{array}$ & 0.00 \\
\hline FL 08 & Ft. Lewis & $\begin{array}{c}2274 \\
3.40 \\
\end{array}$ & $\begin{array}{c}2594 \\
6.00 \\
\end{array}$ & $\begin{array}{c}39386 \\
4.80 \\
\end{array}$ & $\begin{array}{c}981 . \\
50 \\
\end{array}$ & $\begin{array}{c}2276 . \\
00 \\
\end{array}$ & 0.00 & $\begin{array}{c}867.1 \\
0 \\
\end{array}$ & $\begin{array}{c}366.0 \\
0 \\
\end{array}$ & $\begin{array}{c}1368 \\
.82 \\
\end{array}$ & $\begin{array}{c}181.9 \\
0 \\
\end{array}$ & $\begin{array}{c}400.0 \\
0 \\
\end{array}$ & $\begin{array}{c}494.6 \\
7 \\
\end{array}$ \\
\hline FL 09 & Ft. Lewis & $\begin{array}{r}2315 \\
8.10 \\
\end{array}$ & $\begin{array}{c}2376 \\
0.00 \\
\end{array}$ & $\begin{array}{c}31270 \\
7.40 \\
\end{array}$ & $\begin{array}{c}986 . \\
50 \\
\end{array}$ & $\begin{array}{c}2274 . \\
00 \\
\end{array}$ & 0.00 & $\begin{array}{c}546.6 \\
0 \\
\end{array}$ & $\begin{array}{c}261.0 \\
0 \\
\end{array}$ & $\begin{array}{c}1506 \\
.56 \\
\end{array}$ & $\begin{array}{c}815.0 \\
0 \\
\end{array}$ & $\begin{array}{c}444.0 \\
0 \\
\end{array}$ & 0.00 \\
\hline FL 20 & Ft. Lewis & $\begin{array}{l}2119 \\
0.70 \\
\end{array}$ & $\begin{array}{l}4001 \\
8.00 \\
\end{array}$ & $\begin{array}{c}54002 \\
2.20 \\
\end{array}$ & $\begin{array}{c}563 . \\
80 \\
\end{array}$ & $\begin{array}{c}1605 . \\
00\end{array}$ & 0.00 & $\begin{array}{c}1024 . \\
80 \\
\end{array}$ & $\begin{array}{c}578.0 \\
0 \\
\end{array}$ & $\begin{array}{c}2856 \\
.42 \\
\end{array}$ & $\begin{array}{c}644.2 \\
0 \\
\end{array}$ & $\begin{array}{c}586.0 \\
0 \\
\end{array}$ & $\begin{array}{c}761.0 \\
4 \\
\end{array}$ \\
\hline FL 21 & Ft. Lewis & $\begin{array}{c}2421 \\
5.50\end{array}$ & $\begin{array}{c}6366 \\
6.00\end{array}$ & $\begin{array}{l}10885 \\
18.00\end{array}$ & $\begin{array}{c}763 . \\
20 \\
\end{array}$ & $\begin{array}{c}2199 . \\
00\end{array}$ & 0.00 & $\begin{array}{c}582.9 \\
0\end{array}$ & $\begin{array}{c}685.0 \\
0\end{array}$ & $\begin{array}{c}1837 \\
.13\end{array}$ & $\begin{array}{c}1247 . \\
90\end{array}$ & $\begin{array}{c}807.0 \\
0\end{array}$ & 30.44 \\
\hline FL 22 & Ft. Lewis & $\begin{array}{l}2281 \\
6.70\end{array}$ & $\begin{array}{c}4535 \\
4.00\end{array}$ & $\begin{array}{c}70323 \\
9.80\end{array}$ & $\begin{array}{c}529 . \\
40\end{array}$ & $\begin{array}{c}1350 . \\
00\end{array}$ & 0.00 & $\begin{array}{c}731.5 \\
0\end{array}$ & $\begin{array}{c}567.0 \\
0\end{array}$ & $\begin{array}{c}3724 \\
.19\end{array}$ & $\begin{array}{c}616.5 \\
0\end{array}$ & $\begin{array}{c}620.0 \\
0\end{array}$ & 0.00 \\
\hline FL 23 & Ft. Lewis & $\begin{array}{l}2590 \\
5.60 \\
\end{array}$ & $\begin{array}{c}4358 \\
3.00 \\
\end{array}$ & $\begin{array}{c}72293 \\
8.90 \\
\end{array}$ & $\begin{array}{c}417 . \\
00 \\
\end{array}$ & - & 0.00 & $\begin{array}{c}1818 . \\
90 \\
\end{array}$ & $\begin{array}{c}576.0 \\
0 \\
\end{array}$ & $\begin{array}{c}1685 \\
.62 \\
\end{array}$ & $\begin{array}{c}160.5 \\
0 \\
\end{array}$ & $\begin{array}{c}573.0 \\
0 \\
\end{array}$ & 0.00 \\
\hline FL 24 & Ft. Lewis & $\begin{array}{c}2306 \\
9.40 \\
\end{array}$ & $\begin{array}{c}5279 \\
1.00 \\
\end{array}$ & $\begin{array}{c}60798 \\
5.20 \\
\end{array}$ & $\begin{array}{c}849 . \\
00 \\
\end{array}$ & $\begin{array}{c}1686 . \\
00\end{array}$ & 0.00 & $\begin{array}{c}935.6 \\
0 \\
\end{array}$ & $\begin{array}{c}609.0 \\
0 \\
\end{array}$ & $\begin{array}{c}1837 \\
.13 \\
\end{array}$ & $\begin{array}{c}221.6 \\
0 \\
\end{array}$ & $\begin{array}{c}718.0 \\
0 \\
\end{array}$ & $\begin{array}{c}121.7 \\
7 \\
\end{array}$ \\
\hline FL 25 & Ft. Lewis & $\begin{array}{r}2379 \\
6.10 \\
\end{array}$ & $\begin{array}{r}4369 \\
4.00 \\
\end{array}$ & $\begin{array}{c}61367 \\
9.60 \\
\end{array}$ & $\begin{array}{c}782 . \\
30 \\
\end{array}$ & $\begin{array}{c}1788 . \\
00\end{array}$ & 0.00 & $\begin{array}{c}801.2 \\
0 \\
\end{array}$ & $\begin{array}{c}540.0 \\
0 \\
\end{array}$ & $\begin{array}{c}1878 \\
.46 \\
\end{array}$ & $\begin{array}{c}283.3 \\
0 \\
\end{array}$ & $\begin{array}{c}660.0 \\
0 \\
\end{array}$ & 0.00 \\
\hline FL 26 & Ft. Lewis & $\begin{array}{l}2178 \\
2.00\end{array}$ & $\begin{array}{c}4364 \\
5.00\end{array}$ & $\begin{array}{c}62270 \\
7.40\end{array}$ & $\begin{array}{c}331 . \\
90\end{array}$ & - & 0.00 & $\begin{array}{c}876.1 \\
0\end{array}$ & $\begin{array}{c}642.0 \\
0\end{array}$ & $\begin{array}{c}905 . \\
09\end{array}$ & $\begin{array}{c}528.1 \\
0\end{array}$ & $\begin{array}{c}470.0 \\
0\end{array}$ & 0.00 \\
\hline FL 27 & Ft. Lewis & $\begin{array}{l}1800 \\
2.00\end{array}$ & $\begin{array}{l}3541 \\
8.00\end{array}$ & $\begin{array}{c}68361 \\
0.20\end{array}$ & $\begin{array}{c}173 . \\
00\end{array}$ & _ & 0.00 & $\begin{array}{c}341.2 \\
0\end{array}$ & $\begin{array}{c}502.0 \\
0\end{array}$ & $\begin{array}{c}2071 \\
.29\end{array}$ & $\begin{array}{c}103.9 \\
0\end{array}$ & $\begin{array}{c}494.0 \\
0\end{array}$ & 0.00 \\
\hline FL 28 & Ft. Lewis & $\begin{array}{l}2035 \\
4.60 \\
\end{array}$ & $\begin{array}{c}3164 \\
1.00 \\
\end{array}$ & $\begin{array}{c}94384 \\
1.70 \\
\end{array}$ & $\begin{array}{c}394 . \\
70 \\
\end{array}$ & $\begin{array}{c}1638 . \\
00\end{array}$ & 0.00 & $\begin{array}{c}850.0 \\
0 \\
\end{array}$ & $\begin{array}{c}497.0 \\
0 \\
\end{array}$ & $\begin{array}{c}3939 \\
.98 \\
\end{array}$ & $\begin{array}{c}389.9 \\
0 \\
\end{array}$ & $\begin{array}{c}570.0 \\
0 \\
\end{array}$ & $\begin{array}{c}859.9 \\
7 \\
\end{array}$ \\
\hline FL 29 & Ft. Lewis & $\begin{array}{l}2461 \\
7.70\end{array}$ & $\begin{array}{c}4517 \\
3.00\end{array}$ & $\begin{array}{c}70094 \\
8.10\end{array}$ & $\begin{array}{c}1155 \\
.90\end{array}$ & - & 0.00 & $\begin{array}{c}798.4 \\
0\end{array}$ & $\begin{array}{c}657.0 \\
0\end{array}$ & $\begin{array}{c}2796 \\
.73\end{array}$ & $\begin{array}{c}258.9 \\
0\end{array}$ & $\begin{array}{c}597.0 \\
0\end{array}$ & 0.00 \\
\hline FL 30 & Ft. Lewis & $\begin{array}{r}2750 \\
6.10 \\
\end{array}$ & $\begin{array}{r}4172 \\
0.00 \\
\end{array}$ & $\begin{array}{c}73865 \\
6.50 \\
\end{array}$ & $\begin{array}{c}430 . \\
30 \\
\end{array}$ & $\begin{array}{c}737.0 \\
0 \\
\end{array}$ & 0.00 & $\begin{array}{c}732.5 \\
0 \\
\end{array}$ & $\begin{array}{c}403.0 \\
0 \\
\end{array}$ & $\begin{array}{c}2998 \\
.75 \\
\end{array}$ & $\begin{array}{c}1200 . \\
70\end{array}$ & $\begin{array}{c}599.0 \\
0 \\
\end{array}$ & 0.00 \\
\hline FL 31 & Ft. Lewis & $\begin{array}{c}1394 \\
5.00\end{array}$ & $\begin{array}{c}3936 \\
6.00\end{array}$ & $\begin{array}{c}39944 \\
3.50\end{array}$ & $\begin{array}{c}39.6 \\
0\end{array}$ & - & 0.00 & $\begin{array}{c}440.1 \\
0\end{array}$ & $\begin{array}{c}448.0 \\
0\end{array}$ & $\begin{array}{c}321 . \\
98\end{array}$ & 93.00 & $\begin{array}{c}334.0 \\
0\end{array}$ & 0.00 \\
\hline FL 36 & Ft. Lewis & $\begin{array}{l}2056 \\
5.40\end{array}$ & $\begin{array}{l}5581 \\
4.00\end{array}$ & $\begin{array}{c}60495 \\
2.80\end{array}$ & $\begin{array}{c}248 . \\
20\end{array}$ & - & 0.00 & $\begin{array}{c}621.4 \\
0\end{array}$ & $\begin{array}{c}645.0 \\
0\end{array}$ & $\begin{array}{c}2714 \\
.09\end{array}$ & $\begin{array}{c}626.2 \\
0\end{array}$ & $\begin{array}{c}620.0 \\
0\end{array}$ & 0.00 \\
\hline FL 37 & Ft. Lewis & $\begin{array}{l}1953 \\
0.80 \\
\end{array}$ & $\begin{array}{c}5944 \\
4.00 \\
\end{array}$ & $\begin{array}{c}58050 \\
8.30 \\
\end{array}$ & $\begin{array}{c}352 . \\
60 \\
\end{array}$ & - & 0.00 & $\begin{array}{c}1470 . \\
80 \\
\end{array}$ & $\begin{array}{c}700.0 \\
0 \\
\end{array}$ & $\begin{array}{c}1713 \\
.17 \\
\end{array}$ & $\begin{array}{c}496.2 \\
0 \\
\end{array}$ & $\begin{array}{c}634.0 \\
0 \\
\end{array}$ & 0.00 \\
\hline FL 38 & Ft. Lewis & $\begin{array}{c}1832 \\
9.30 \\
\end{array}$ & $\begin{array}{c}8731 \\
5.00 \\
\end{array}$ & $\begin{array}{c}72203 \\
6.10 \\
\end{array}$ & $\begin{array}{c}1021 \\
.40 \\
\end{array}$ & $\begin{array}{c}2507 . \\
00\end{array}$ & 0.00 & $\begin{array}{c}690.8 \\
0 \\
\end{array}$ & $\begin{array}{c}641.0 \\
0\end{array}$ & $\begin{array}{c}2677 \\
.35 \\
\end{array}$ & $\begin{array}{c}944.8 \\
0 \\
\end{array}$ & $\begin{array}{c}935.0 \\
0 \\
\end{array}$ & 0.00 \\
\hline FL 39 & Ft. Lewis & $\begin{array}{c}2920 . \\
60\end{array}$ & $\begin{array}{c}3999 \\
5.00\end{array}$ & $\begin{array}{c}74215 \\
1.90\end{array}$ & $\begin{array}{c}136 . \\
50\end{array}$ & $\begin{array}{c}1202 . \\
00\end{array}$ & 0.00 & $\begin{array}{c}106.5 \\
0\end{array}$ & $\begin{array}{c}305.0 \\
0\end{array}$ & $\begin{array}{c}2957 \\
.43\end{array}$ & $\begin{array}{c}123.8 \\
0\end{array}$ & $\begin{array}{c}619.0 \\
0\end{array}$ & 0.00 \\
\hline FL 40 & Ft. Lewis & $\begin{array}{c}2185 \\
5.00 \\
\end{array}$ & $\begin{array}{c}5655 \\
0.00 \\
\end{array}$ & $\begin{array}{c}68286 \\
9.40 \\
\end{array}$ & $\begin{array}{c}116 . \\
50 \\
\end{array}$ & - & 0.00 & $\begin{array}{c}1047 . \\
30 \\
\end{array}$ & $\begin{array}{c}683.0 \\
0 \\
\end{array}$ & $\begin{array}{c}1534 \\
.10 \\
\end{array}$ & $\begin{array}{c}897.8 \\
0 \\
\end{array}$ & $\begin{array}{c}471.0 \\
0 \\
\end{array}$ & 0.00 \\
\hline FL 41 & Ft. Lewis & $\begin{array}{c}5626 . \\
70 \\
\end{array}$ & $\begin{array}{c}8807 . \\
00\end{array}$ & $\begin{array}{c}38722 \\
1.30 \\
\end{array}$ & - & - & 0.00 & $\begin{array}{c}112.0 \\
0 \\
\end{array}$ & 93.00 & $\begin{array}{c}2571 \\
.75 \\
\end{array}$ & $\begin{array}{c}109.7 \\
0 \\
\end{array}$ & $\begin{array}{c}172.0 \\
0 \\
\end{array}$ & $\begin{array}{c}334.8 \\
6 \\
\end{array}$ \\
\hline FL 42 & Ft. Lewis & $\begin{array}{l}1071 \\
3.10\end{array}$ & $\begin{array}{c}2068 \\
4.00\end{array}$ & $\begin{array}{c}36650 \\
3.70\end{array}$ & 6.60 & - & 0.00 & $\begin{array}{c}250.6 \\
0\end{array}$ & $\begin{array}{c}323.0 \\
0\end{array}$ & $\begin{array}{c}1295 \\
.35\end{array}$ & $\begin{array}{c}317.0 \\
0\end{array}$ & $\begin{array}{c}287.0 \\
0\end{array}$ & 0.00 \\
\hline FL 43 & Ft. Lewis & $\begin{array}{l}1899 \\
3.20 \\
\end{array}$ & $\begin{array}{r}4777 \\
0.00\end{array}$ & $\begin{array}{c}70499 \\
9.10\end{array}$ & $\begin{array}{c}203 . \\
80\end{array}$ & 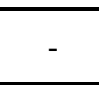 & 0.00 & $\begin{array}{c}1304 . \\
80\end{array}$ & $\begin{array}{c}834.0 \\
0\end{array}$ & $\begin{array}{c}1093 \\
.33\end{array}$ & $\begin{array}{c}128.6 \\
0\end{array}$ & $\begin{array}{c}496.0 \\
0\end{array}$ & 0.00 \\
\hline FL 44 & Ft. Lewis & $\begin{array}{c}2203 \\
7.30\end{array}$ & $\begin{array}{l}7936 \\
2.00\end{array}$ & $\begin{array}{c}72270 \\
7.40\end{array}$ & $\begin{array}{c}377 . \\
30\end{array}$ & 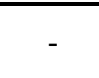 & 0.00 & $\begin{array}{c}1115 . \\
10\end{array}$ & $\begin{array}{c}1195 . \\
00\end{array}$ & $\begin{array}{c}1681 \\
.03\end{array}$ & $\begin{array}{c}469.5 \\
0\end{array}$ & $\begin{array}{c}652.0 \\
0\end{array}$ & $\begin{array}{c}403.3 \\
5\end{array}$ \\
\hline FL 45 & Ft. Lewis & $\begin{array}{c}2014 \\
6.50 \\
\end{array}$ & $\begin{array}{r}4881 \\
8.00 \\
\end{array}$ & $\begin{array}{c}53583 \\
2.40 \\
\end{array}$ & $\begin{array}{c}384 . \\
80 \\
\end{array}$ & $\begin{array}{c}819.0 \\
0 \\
\end{array}$ & 0.00 & $\begin{array}{c}594.6 \\
0 \\
\end{array}$ & $\begin{array}{c}311.0 \\
0 \\
\end{array}$ & $\begin{array}{c}905 . \\
09 \\
\end{array}$ & $\begin{array}{c}657.6 \\
0 \\
\end{array}$ & $\begin{array}{c}734.0 \\
0 \\
\end{array}$ & 0.00 \\
\hline FL 46 & Ft. Lewis & $\begin{array}{c}2206 \\
6.50\end{array}$ & $\begin{array}{c}5648 \\
7.00\end{array}$ & $\begin{array}{c}20367 \\
9.60\end{array}$ & $\begin{array}{c}343 . \\
00\end{array}$ & $\begin{array}{c}1943 . \\
00\end{array}$ & 0.00 & $\begin{array}{c}433.7 \\
0\end{array}$ & $\begin{array}{c}332.0 \\
0\end{array}$ & $\begin{array}{c}450 . \\
54\end{array}$ & $\begin{array}{c}1594 . \\
50\end{array}$ & $\begin{array}{c}810.0 \\
0\end{array}$ & 0.00 \\
\hline FL 47 & Ft. Lewis & $\begin{array}{c}3704 . \\
30 \\
\end{array}$ & $\begin{array}{c}8741 . \\
00 \\
\end{array}$ & $\begin{array}{c}58332 \\
.41 \\
\end{array}$ & $\begin{array}{c}15.9 \\
0 \\
\end{array}$ & 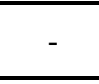 & 0.00 & $\begin{array}{c}148.9 \\
0\end{array}$ & $\begin{array}{c}100.0 \\
0 \\
\end{array}$ & $\begin{array}{c}1033 \\
.65 \\
\end{array}$ & 79.00 & $\begin{array}{c}252.0 \\
0 \\
\end{array}$ & 0.00 \\
\hline FL 48 & Ft. Lewis & $\begin{array}{c}3250 . \\
70\end{array}$ & $\begin{array}{c}7413 . \\
00\end{array}$ & $\begin{array}{c}25462 \\
.04\end{array}$ & $\begin{array}{c}68.2 \\
0\end{array}$ & $\begin{array}{c}225.0 \\
0\end{array}$ & 0.00 & 48.40 & $\begin{array}{c}118.0 \\
0\end{array}$ & $\begin{array}{c}1029 \\
.05\end{array}$ & 64.10 & $\begin{array}{c}220.0 \\
0\end{array}$ & 0.00 \\
\hline
\end{tabular}




\begin{tabular}{|c|c|c|c|c|c|c|c|c|c|c|c|c|c|}
\hline FL 49 & Ft. Lewis & $\begin{array}{c}8778 . \\
20\end{array}$ & $\begin{array}{l}2415 \\
3.00\end{array}$ & $\begin{array}{c}21108 \\
7.00\end{array}$ & $\begin{array}{c}220 . \\
40\end{array}$ & & 0.00 & $\begin{array}{c}279.4 \\
0\end{array}$ & $\begin{array}{c}475.0 \\
0\end{array}$ & $\begin{array}{c}1644 \\
.30\end{array}$ & $\begin{array}{c}217.0 \\
0\end{array}$ & $\begin{array}{c}494.0 \\
0\end{array}$ & $\begin{array}{c}1971 . \\
08\end{array}$ \\
\hline FL 50 & Ft. Lewis & $\begin{array}{c}7478 . \\
70 \\
\end{array}$ & $\begin{array}{l}2277 \\
0.00 \\
\end{array}$ & $\begin{array}{c}11900 \\
3.70 \\
\end{array}$ & $\begin{array}{c}105 . \\
70 \\
\end{array}$ & - & 0.00 & $\begin{array}{c}216.8 \\
0 \\
\end{array}$ & $\begin{array}{c}384.0 \\
0 \\
\end{array}$ & 0.00 & 44.40 & $\begin{array}{c}210.0 \\
0 \\
\end{array}$ & 0.00 \\
\hline FL 51 & Ft. Lewis & $\begin{array}{c}199.7 \\
0 \\
\end{array}$ & $\begin{array}{c}868.0 \\
0 \\
\end{array}$ & 0.00 & - & - & 0.00 & - & - & $\begin{array}{c}303 . \\
62 \\
\end{array}$ & 23.50 & 74.00 & 0.00 \\
\hline FL 52 & Ft. Lewis & $\begin{array}{c}314.2 \\
0 \\
\end{array}$ & $\begin{array}{c}667.0 \\
0 \\
\end{array}$ & 0.00 & - & & 0.00 & - & - & $\begin{array}{c}3260 \\
.46 \\
\end{array}$ & 20.30 & 65.00 & $\begin{array}{c}433.7 \\
9 \\
\end{array}$ \\
\hline FL 53 & Ft. Lewis & 83.10 & $\begin{array}{c}262.0 \\
0\end{array}$ & $\begin{array}{c}68540 \\
.74\end{array}$ & - & - & 0.00 & - & - & $\begin{array}{c}932 . \\
63\end{array}$ & 24.80 & 66.00 & $\begin{array}{c}167.4 \\
3\end{array}$ \\
\hline FL 54 & Ft. Lewis & $\begin{array}{c}105.1 \\
0\end{array}$ & $\begin{array}{c}227.0 \\
0\end{array}$ & 0.00 & - & & 0.00 & - & - & $\begin{array}{c}1722 \\
.35\end{array}$ & 27.70 & 67.00 & $\begin{array}{c}426.1 \\
8\end{array}$ \\
\hline FL 55 & Ft. Lewis & 64.60 & $\begin{array}{c}135.0 \\
0 \\
\end{array}$ & $\begin{array}{c}12094 \\
8.10 \\
\end{array}$ & - & - & 0.00 & - & - & 0.00 & 48.30 & 95.00 & 0.00 \\
\hline FL 56 & Ft. Lewis & 53.70 & $\begin{array}{c}119.0 \\
0 \\
\end{array}$ & $\begin{array}{c}54721 \\
.30 \\
\end{array}$ & - & - & 0.00 & - & - & $\begin{array}{c}110 . \\
78 \\
\end{array}$ & 45.60 & 80.00 & 0.00 \\
\hline FL 58 & Ft. Lewis & & $\begin{array}{l}6738 \\
7.00\end{array}$ & $\begin{array}{c}54349 \\
4.40\end{array}$ & $\begin{array}{c}409 . \\
30\end{array}$ & $\begin{array}{c}848.0 \\
0\end{array}$ & 0.00 & $\begin{array}{c}532.9 \\
0\end{array}$ & $\begin{array}{c}669.0 \\
0\end{array}$ & $\begin{array}{c}1800 \\
.40\end{array}$ & $\begin{array}{c}267.6 \\
0\end{array}$ & $\begin{array}{c}778.0 \\
0\end{array}$ & 0.00 \\
\hline FL 59 & Ft. Lewis & $\begin{array}{l}1949 \\
8.60\end{array}$ & $\begin{array}{r}6554 \\
4.00\end{array}$ & $\begin{array}{c}85647 \\
.22\end{array}$ & $\begin{array}{c}485 . \\
50\end{array}$ & $\begin{array}{c}980.0 \\
0\end{array}$ & 0.00 & $\begin{array}{c}861.0 \\
0\end{array}$ & $\begin{array}{c}723.0 \\
0\end{array}$ & $\begin{array}{c}276 . \\
07\end{array}$ & $\begin{array}{c}812.6 \\
0\end{array}$ & $\begin{array}{c}688.0 \\
0\end{array}$ & 0.00 \\
\hline FL 60 & Ft. Lewis & $\begin{array}{l}2018 \\
4.30\end{array}$ & $\begin{array}{l}7113 \\
3.00\end{array}$ & $\begin{array}{c}63817 \\
0.40\end{array}$ & $\begin{array}{c}559 . \\
70\end{array}$ & $\begin{array}{c}1110 . \\
00\end{array}$ & 0.00 & $\begin{array}{c}1096 . \\
30\end{array}$ & $\begin{array}{c}756.0 \\
0\end{array}$ & $\begin{array}{c}2521 \\
.25\end{array}$ & $\begin{array}{c}1210 . \\
10\end{array}$ & $\begin{array}{c}844.0 \\
0\end{array}$ & $\begin{array}{c}799.0 \\
9\end{array}$ \\
\hline FL 61 & Ft. Lewis & $\begin{array}{l}1675 \\
5.30 \\
\end{array}$ & $\begin{array}{l}7019 \\
6.00 \\
\end{array}$ & $\begin{array}{c}65374 \\
9.10 \\
\end{array}$ & $\begin{array}{c}493 . \\
80 \\
\end{array}$ & $\begin{array}{c}835.0 \\
0 \\
\end{array}$ & 0.00 & $\begin{array}{c}832.9 \\
0 \\
\end{array}$ & $\begin{array}{c}892.0 \\
0 \\
\end{array}$ & $\begin{array}{c}1814 \\
.18 \\
\end{array}$ & $\begin{array}{c}1183 . \\
70\end{array}$ & $\begin{array}{c}793.0 \\
0\end{array}$ & $\begin{array}{c}776.2 \\
6\end{array}$ \\
\hline FL 64 & Ft. Lewis & $\begin{array}{c}4649 . \\
70\end{array}$ & $\begin{array}{c}6802 . \\
00\end{array}$ & $\begin{array}{c}20847 \\
1.30\end{array}$ & 6.80 & - & 0.00 & 79.00 & $\begin{array}{c}125.0 \\
0 \\
\end{array}$ & $\begin{array}{c}1405 \\
.55 \\
\end{array}$ & 63.90 & $\begin{array}{c}186.0 \\
0 \\
\end{array}$ & 0.00 \\
\hline FL 65 & Ft. Lewis & $\begin{array}{l}1959 \\
9.30 \\
\end{array}$ & $\begin{array}{l}6303 \\
2.00 \\
\end{array}$ & $\begin{array}{c}57455 \\
9.30 \\
\end{array}$ & $\begin{array}{c}244 . \\
30 \\
\end{array}$ & - & 0.00 & $\begin{array}{c}1255 . \\
10 \\
\end{array}$ & $\begin{array}{c}884.0 \\
0 \\
\end{array}$ & $\begin{array}{c}1304 \\
.54 \\
\end{array}$ & $\begin{array}{c}492.9 \\
0 \\
\end{array}$ & $\begin{array}{c}578.0 \\
0 \\
\end{array}$ & $\begin{array}{c}1240 . \\
49 \\
\end{array}$ \\
\hline FL 68 & Ft. Lewis & $\begin{array}{c}2038 \\
5.40 \\
\end{array}$ & $\begin{array}{l}7032 \\
1.00 \\
\end{array}$ & $\begin{array}{c}72791 \\
5.70 \\
\end{array}$ & $\begin{array}{c}671 . \\
10 \\
\end{array}$ & $\begin{array}{c}1384 . \\
00\end{array}$ & 0.00 & $\begin{array}{c}852.4 \\
0 \\
\end{array}$ & $\begin{array}{c}755.0 \\
0 \\
\end{array}$ & $\begin{array}{c}1671 \\
.85 \\
\end{array}$ & $\begin{array}{c}322.0 \\
0 \\
\end{array}$ & $\begin{array}{c}833.0 \\
0 \\
\end{array}$ & $\begin{array}{c}2404 . \\
87 \\
\end{array}$ \\
\hline FL 70 & Ft. Lewis & $\begin{array}{l}2056 \\
2.30 \\
\end{array}$ & $\begin{array}{l}7132 \\
7.00 \\
\end{array}$ & $\begin{array}{c}64530 \\
0.00 \\
\end{array}$ & $\begin{array}{c}419 . \\
50 \\
\end{array}$ & 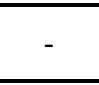 & 0.00 & $\begin{array}{c}1314 . \\
10 \\
\end{array}$ & $\begin{array}{c}1221 . \\
00\end{array}$ & $\begin{array}{l}1437 \\
.69 \\
\end{array}$ & $\begin{array}{c}182.1 \\
0 \\
\end{array}$ & $\begin{array}{c}751.0 \\
0 \\
\end{array}$ & 0.00 \\
\hline $\begin{array}{c}\text { FTWW } \\
\text { B09a }\end{array}$ & $\begin{array}{c}\text { Ft. } \\
\text { Wainwright }\end{array}$ & - & 10.00 & 0.00 & - & - & 0.00 & - & - & $\begin{array}{c}3889 \\
.48 \\
\end{array}$ & 19.00 & 59.00 & $\begin{array}{c}159.8 \\
2 \\
\end{array}$ \\
\hline $\begin{array}{l}\text { FTWW } \\
\text { B09b }\end{array}$ & $\begin{array}{c}\text { Ft. } \\
\text { Wainwright }\end{array}$ & 3.70 & 15.00 & 0.00 & - & - & 0.00 & - & - & $\begin{array}{c}2452 \\
.38\end{array}$ & 22.90 & 65.00 & 0.00 \\
\hline $\begin{array}{c}\text { FTWW } \\
\text { B09c } \\
\end{array}$ & $\begin{array}{c}\text { Ft. } \\
\text { Wainwright }\end{array}$ & 70.00 & 19.00 & 0.00 & - & - & 0.00 & - & - & $\begin{array}{c}3090 \\
.58 \\
\end{array}$ & 24.20 & 64.00 & 0.00 \\
\hline $\begin{array}{c}\text { FTWW } \\
\text { B10a }\end{array}$ & $\begin{array}{c}\text { Ft. } \\
\text { Wainwright }\end{array}$ & $\begin{array}{c}642.0 \\
0 \\
\end{array}$ & $\begin{array}{c}709.0 \\
0 \\
\end{array}$ & 0.00 & - & - & 0.00 & 8.90 & - & $\begin{array}{c}2539 \\
.61 \\
\end{array}$ & 29.00 & 76.00 & 0.00 \\
\hline $\begin{array}{l}\text { FTWW } \\
\text { B10b }\end{array}$ & $\begin{array}{c}\text { Ft. } \\
\text { Wainwright }\end{array}$ & $\begin{array}{c}1832 . \\
90 \\
\end{array}$ & $\begin{array}{c}1017 . \\
00\end{array}$ & $\begin{array}{c}85855 \\
.56 \\
\end{array}$ & - & & 0.00 & 3.40 & - & $\begin{array}{c}2851 \\
.83 \\
\end{array}$ & 59.30 & 77.00 & 38.05 \\
\hline $\begin{array}{l}\text { FTWW } \\
\text { B10c }\end{array}$ & $\begin{array}{c}\text { Ft. } \\
\text { Wainwright }\end{array}$ & 36.40 & 94.00 & 0.00 & - & - & 0.00 & - & - & $\begin{array}{c}1428 \\
.50\end{array}$ & 24.80 & 61.00 & 0.00 \\
\hline $\begin{array}{c}\text { FTWW } \\
\text { B11a }\end{array}$ & $\begin{array}{c}\text { Ft. } \\
\text { Wainwright }\end{array}$ & 41.00 & 70.00 & 0.00 & - & - & 0.00 & - & - & $\begin{array}{c}1524 \\
.92\end{array}$ & 26.10 & 51.00 & $\begin{array}{c}129.3 \\
8\end{array}$ \\
\hline $\begin{array}{c}\text { FTWW } \\
\text { B11b }\end{array}$ & $\begin{array}{c}\text { Ft. } \\
\text { Wainwright }\end{array}$ & 20.00 & 45.00 & 0.00 & - & _- & 0.00 & - & - & $\begin{array}{c}1524 \\
.92 \\
\end{array}$ & 25.50 & $\begin{array}{c}111.0 \\
0 \\
\end{array}$ & $\begin{array}{c}129.3 \\
8 \\
\end{array}$ \\
\hline $\begin{array}{c}\text { FTWW } \\
\text { B11c }\end{array}$ & $\begin{array}{c}\text { Ft. } \\
\text { Wainwright }\end{array}$ & $\begin{array}{c}142.6 \\
0 \\
\end{array}$ & $\begin{array}{c}332.0 \\
0 \\
\end{array}$ & 0.00 & - & - & 0.00 & - & - & $\begin{array}{c}4940 \\
.90 \\
\end{array}$ & 27.30 & 65.00 & 0.00 \\
\hline $\begin{array}{c}\text { FTWW } \\
\text { B12a }\end{array}$ & $\begin{array}{c}\text { Ft. } \\
\text { Wainwright }\end{array}$ & 67.90 & $\begin{array}{c}148.0 \\
0 \\
\end{array}$ & 0.00 & - & - & 0.00 & - & - & $\begin{array}{c}4288 \\
.93 \\
\end{array}$ & 29.00 & 60.00 & 0.00 \\
\hline $\begin{array}{l}\text { FTWW } \\
\text { B12b }\end{array}$ & $\begin{array}{c}\text { Ft. } \\
\text { Wainwright }\end{array}$ & $\begin{array}{c}130.5 \\
0\end{array}$ & 61.00 & 0.00 & - & - & 0.00 & - & - & $\begin{array}{c}2966 \\
.61\end{array}$ & 27.20 & 61.00 & 0.00 \\
\hline $\begin{array}{c}\text { FTWW } \\
\text { B12c }\end{array}$ & $\begin{array}{c}\text { Ft. } \\
\text { Wainwright }\end{array}$ & 21.70 & $\begin{array}{c}136.0 \\
0\end{array}$ & 0.00 & - & - & 0.00 & - & - & $\begin{array}{c}3007 \\
.93\end{array}$ & 26.70 & 51.00 & 0.00 \\
\hline $\begin{array}{c}\text { FTWW } \\
\text { B13a } \\
\end{array}$ & $\begin{array}{c}\text { Ft. } \\
\text { Wainwright }\end{array}$ & $\begin{array}{c}1589 . \\
20 \\
\end{array}$ & $\begin{array}{c}184.0 \\
0 \\
\end{array}$ & 0.00 & - & - & 0.00 & 2.30 & - & $\begin{array}{c}3379 \\
.83 \\
\end{array}$ & 29.00 & 70.00 & 0.00 \\
\hline
\end{tabular}




\begin{tabular}{|c|c|c|c|c|c|c|c|c|c|c|c|c|c|}
\hline $\begin{array}{l}\text { FTWW } \\
\text { B13b }\end{array}$ & $\begin{array}{c}\text { Ft. } \\
\text { Wainwright }\end{array}$ & 13.80 & $\begin{array}{c}120.0 \\
0\end{array}$ & 0.00 & - & & 0.00 & - & - & $\begin{array}{c}2732 \\
.45\end{array}$ & 4.40 & 52.00 & 0.00 \\
\hline $\begin{array}{c}\text { FTWW } \\
\text { B13c } \\
\end{array}$ & $\begin{array}{c}\text { Ft. } \\
\text { Wainwright }\end{array}$ & 45.20 & 75.00 & 0.00 & - & - & 0.00 & - & - & $\begin{array}{c}3324 \\
.74 \\
\end{array}$ & 27.80 & 57.00 & $\begin{array}{c}350.0 \\
8 \\
\end{array}$ \\
\hline $\begin{array}{c}\text { FTWW } \\
\text { B14a }\end{array}$ & $\begin{array}{c}\text { Ft. } \\
\text { Wainwright }\end{array}$ & 22.40 & $\begin{array}{c}158.0 \\
0\end{array}$ & 0.00 & - & - & 0.00 & - & - & $\begin{array}{c}1965 \\
.69\end{array}$ & 23.60 & 60.00 & 0.00 \\
\hline $\begin{array}{l}\text { FTWW } \\
\text { B14b }\end{array}$ & $\begin{array}{c}\text { Ft. } \\
\text { Wainwright }\end{array}$ & 7.80 & 40.00 & 0.00 & - & - & 0.00 & - & - & $\begin{array}{c}1561 \\
.65 \\
\end{array}$ & 26.10 & 80.00 & 0.00 \\
\hline $\begin{array}{c}\text { FTWW } \\
\text { B14c }\end{array}$ & $\begin{array}{c}\text { Ft. } \\
\text { Wainwright }\end{array}$ & 21.20 & 73.00 & 0.00 & - & - & 0.00 & - & - & $\begin{array}{c}1378 \\
.00\end{array}$ & 25.00 & 62.00 & 0.00 \\
\hline $\begin{array}{c}\text { FTWW } \\
\text { B15a }\end{array}$ & $\begin{array}{c}\text { Ft. } \\
\text { Wainwright }\end{array}$ & $\begin{array}{c}149.8 \\
0\end{array}$ & $\begin{array}{c}115.0 \\
0\end{array}$ & 0.00 & - & - & 0.00 & - & - & $\begin{array}{c}2075 \\
.89\end{array}$ & 28.00 & 74.00 & 0.00 \\
\hline $\begin{array}{c}\text { FTWW } \\
\text { B15b }\end{array}$ & $\begin{array}{c}\text { Ft. } \\
\text { Wainwright }\end{array}$ & - & 26.00 & 0.00 & - & - & 0.00 & - & - & $\begin{array}{c}1074 \\
.97 \\
\end{array}$ & 26.50 & 61.00 & 0.00 \\
\hline $\begin{array}{c}\text { FTWW } \\
\text { B15c }\end{array}$ & $\begin{array}{c}\text { Ft. } \\
\text { Wainwright }\end{array}$ & $\begin{array}{c}394.2 \\
0 \\
\end{array}$ & $\begin{array}{c}568.0 \\
0 \\
\end{array}$ & $\begin{array}{c}26387 \\
.96 \\
\end{array}$ & - & - & 0.00 & - & - & $\begin{array}{c}1309 \\
.13 \\
\end{array}$ & 25.30 & 63.00 & 0.00 \\
\hline $\begin{array}{c}\text { FTWW } \\
\text { B16a }\end{array}$ & $\begin{array}{c}\text { Ft. } \\
\text { Wainwright }\end{array}$ & 3.90 & 22.00 & 0.00 & - & - & 0.00 & - & - & $\begin{array}{c}1318 \\
.31\end{array}$ & 24.20 & 59.00 & 0.00 \\
\hline $\begin{array}{c}\text { FTWW } \\
\text { B16b }\end{array}$ & $\begin{array}{c}\text { Ft. } \\
\text { Wainwright }\end{array}$ & 16.10 & 30.00 & 0.00 & - & - & 0.00 & - & - & $\begin{array}{c}4114 \\
.45 \\
\end{array}$ & 26.80 & 58.00 & 0.00 \\
\hline $\begin{array}{c}\text { FTWW } \\
\text { B16c }\end{array}$ & $\begin{array}{c}\text { Ft. } \\
\text { Wainwright }\end{array}$ & 29.20 & 82.00 & 0.00 & - & - & 0.00 & - & - & $\begin{array}{c}2052 \\
.93\end{array}$ & 25.90 & 59.00 & 0.00 \\
\hline $\begin{array}{l}\text { FTWW } \\
\text { BCK 1a } \\
\end{array}$ & $\begin{array}{c}\text { Ft. } \\
\text { Wainwright }\end{array}$ & $\begin{array}{c}137.5 \\
0 \\
\end{array}$ & $\begin{array}{c}233.0 \\
0 \\
\end{array}$ & 0.00 & - & - & 0.00 & - & - & $\begin{array}{c}1175 \\
.98 \\
\end{array}$ & 41.70 & 90.00 & $\begin{array}{c}951.2 \\
9 \\
\end{array}$ \\
\hline $\begin{array}{l}\text { FTWW } \\
\text { Bck 1c }\end{array}$ & $\begin{array}{c}\text { Ft. } \\
\text { Wainwright }\end{array}$ & $\begin{array}{c}173.1 \\
0\end{array}$ & $\begin{array}{c}484.0 \\
0\end{array}$ & 0.00 & - & - & 0.00 & - & - & 0.00 & 43.20 & 94.00 & $\begin{array}{c}799.0 \\
9 \\
\end{array}$ \\
\hline $\begin{array}{l}\text { FTWW } \\
\text { MI-01 }\end{array}$ & $\begin{array}{c}\text { Ft. } \\
\text { Wainwright }\end{array}$ & $\begin{array}{c}207.4 \\
0\end{array}$ & $\begin{array}{c}422.0 \\
0\end{array}$ & 0.00 & - & - & 0.00 & - & - & $\begin{array}{c}2149 \\
.35 \\
\end{array}$ & 41.60 & 68.00 & 0.00 \\
\hline $\begin{array}{l}\text { FTWW } \\
\text { MI-02 }\end{array}$ & $\begin{array}{c}\text { Ft. } \\
\text { Wainwright }\end{array}$ & $\begin{array}{c}263.3 \\
0 \\
\end{array}$ & $\begin{array}{c}275.0 \\
0 \\
\end{array}$ & 0.00 & - & - & 0.00 & - & - & $\begin{array}{c}3255 \\
.87 \\
\end{array}$ & 29.30 & 52.00 & 0.00 \\
\hline $\begin{array}{l}\text { FTWW } \\
\text { MI-03 }\end{array}$ & $\begin{array}{c}\text { Ft. } \\
\text { Wainwright }\end{array}$ & $\begin{array}{c}216.8 \\
0\end{array}$ & $\begin{array}{c}760.0 \\
0\end{array}$ & 0.00 & - & - & 0.00 & - & - & $\begin{array}{c}2500 \\
.59\end{array}$ & 28.80 & 62.00 & 0.00 \\
\hline $\begin{array}{l}\text { FTWW } \\
\text { MI-04 }\end{array}$ & $\begin{array}{c}\text { Ft. } \\
\text { Wainwright }\end{array}$ & $\begin{array}{c}401.6 \\
0 \\
\end{array}$ & $\begin{array}{c}287.0 \\
0 \\
\end{array}$ & 0.00 & - & - & 0.00 & 3.60 & - & $\begin{array}{c}1726 \\
.94 \\
\end{array}$ & 28.00 & 66.00 & 0.00 \\
\hline $\begin{array}{l}\text { FTWW } \\
\text { MI-05 }\end{array}$ & $\begin{array}{c}\text { Ft. } \\
\text { Wainwright }\end{array}$ & $\begin{array}{c}235.6 \\
0\end{array}$ & $\begin{array}{c}299.0 \\
0\end{array}$ & 0.00 & - & - & 0.00 & - & - & $\begin{array}{c}3187 \\
.00\end{array}$ & 41.20 & 71.00 & 68.49 \\
\hline $\begin{array}{l}\text { FTWW } \\
\text { MI-06 }\end{array}$ & $\begin{array}{c}\text { Ft. } \\
\text { Wainwright }\end{array}$ & $\begin{array}{c}192.6 \\
0 \\
\end{array}$ & $\begin{array}{c}278.0 \\
0 \\
\end{array}$ & 0.00 & - & - & 0.00 & - & - & $\begin{array}{c}4265 \\
.97 \\
\end{array}$ & 28.50 & 72.00 & 0.00 \\
\hline $\begin{array}{l}\text { FTWW } \\
\text { MI-15 } \\
\end{array}$ & $\begin{array}{c}\text { Ft. } \\
\text { Wainwright }\end{array}$ & $\begin{array}{c}167.6 \\
0 \\
\end{array}$ & $\begin{array}{c}343.0 \\
0 \\
\end{array}$ & 0.00 & - & - & 0.00 & - & - & $\begin{array}{c}2231 \\
.99 \\
\end{array}$ & 39.40 & 66.00 & 0.00 \\
\hline $\begin{array}{l}\text { FTWW } \\
\text { MI-17 }\end{array}$ & $\begin{array}{c}\text { Ft. } \\
\text { Wainwright }\end{array}$ & $\begin{array}{c}209.9 \\
0 \\
\end{array}$ & $\begin{array}{c}605.0 \\
0 \\
\end{array}$ & 0.00 & - & - & 0.00 & - & - & $\begin{array}{c}3205 \\
.36 \\
\end{array}$ & 29.00 & 63.00 & 0.00 \\
\hline $\begin{array}{c}\text { FTWW } \\
\text { MI-18 }\end{array}$ & $\begin{array}{c}\text { Ft. } \\
\text { Wainwright }\end{array}$ & $\begin{array}{c}396.3 \\
0\end{array}$ & $\begin{array}{c}365.0 \\
0\end{array}$ & 0.00 & - & - & 0.00 & 3.00 & - & $\begin{array}{c}3343 \\
.10\end{array}$ & 27.90 & 56.00 & 0.00 \\
\hline $\begin{array}{c}\text { FTWW } \\
\text { MI-19 }\end{array}$ & $\begin{array}{c}\text { Ft. } \\
\text { Wainwright }\end{array}$ & $\begin{array}{c}248.0 \\
0 \\
\end{array}$ & $\begin{array}{c}771.0 \\
0 \\
\end{array}$ & 0.00 & - & - & 0.00 & - & 69.00 & $\begin{array}{c}3017 \\
.12 \\
\end{array}$ & 33.80 & $\begin{array}{c}160.0 \\
0 \\
\end{array}$ & 0.00 \\
\hline $\begin{array}{c}\text { FTWW } \\
\text { MI-20 }\end{array}$ & $\begin{array}{c}\text { Ft. } \\
\text { Wainwright }\end{array}$ & $\begin{array}{c}544.5 \\
0 \\
\end{array}$ & $\begin{array}{c}455.0 \\
0 \\
\end{array}$ & 0.00 & - & - & 0.00 & 5.80 & - & $\begin{array}{c}1974 \\
.88 \\
\end{array}$ & 35.10 & 92.00 & 68.49 \\
\hline $\begin{array}{l}\text { FTWW } \\
\text { MI-21 }\end{array}$ & $\begin{array}{c}\text { Ft. } \\
\text { Wainwright }\end{array}$ & $\begin{array}{c}364.4 \\
0 \\
\end{array}$ & $\begin{array}{c}721.0 \\
0 \\
\end{array}$ & 0.00 & - & - & 0.00 & - & - & $\begin{array}{c}3512 \\
.98 \\
\end{array}$ & 32.50 & 77.00 & 0.00 \\
\hline $\begin{array}{l}\text { FTWW } \\
\text { MI-22 }\end{array}$ & $\begin{array}{c}\text { Ft. } \\
\text { Wainwright }\end{array}$ & $\begin{array}{c}432.1 \\
0 \\
\end{array}$ & $\begin{array}{c}550.0 \\
0 \\
\end{array}$ & 0.00 & - & - & 0.00 & 3.80 & - & $\begin{array}{c}1446 \\
.87 \\
\end{array}$ & 36.90 & 58.00 & $\begin{array}{c}281.5 \\
8 \\
\end{array}$ \\
\hline $\begin{array}{l}\text { FTWW } \\
\text { MI-23 }\end{array}$ & $\begin{array}{c}\text { Ft. } \\
\text { Wainwright }\end{array}$ & $\begin{array}{c}331.3 \\
0\end{array}$ & $\begin{array}{c}838.0 \\
0\end{array}$ & 0.00 & - & - & 0.00 & - & - & $\begin{array}{c}2760 \\
.00\end{array}$ & 32.10 & 66.00 & 0.00 \\
\hline $\begin{array}{l}\text { FTWW } \\
\text { MI-24 }\end{array}$ & $\begin{array}{c}\text { Ft. } \\
\text { Wainwright }\end{array}$ & $\begin{array}{c}561.1 \\
0\end{array}$ & $\begin{array}{c}413.0 \\
0\end{array}$ & 0.00 & - & - & 0.00 & 5.90 & - & $\begin{array}{c}3779 \\
.28\end{array}$ & 31.70 & 59.00 & $\begin{array}{c}395.7 \\
4\end{array}$ \\
\hline $\begin{array}{l}\text { FTWW } \\
\text { MI-25 }\end{array}$ & $\begin{array}{c}\text { Ft. } \\
\text { Wainwright }\end{array}$ & $\begin{array}{c}298.5 \\
0 \\
\end{array}$ & $\begin{array}{c}598.0 \\
0 \\
\end{array}$ & $\begin{array}{c}8124 . \\
07 \\
\end{array}$ & - & - & 0.00 & 2.10 & - & $\begin{array}{c}1699 \\
.39 \\
\end{array}$ & 30.20 & 69.00 & $\begin{array}{c}1027 . \\
40 \\
\end{array}$ \\
\hline
\end{tabular}




\begin{tabular}{|c|c|c|c|c|c|c|c|c|c|c|c|c|c|}
\hline $\begin{array}{l}\text { FTWW } \\
\text { MI-27 }\end{array}$ & $\begin{array}{c}\text { Ft. } \\
\text { Wainwright }\end{array}$ & $\begin{array}{c}458.4 \\
0\end{array}$ & $\begin{array}{c}325.0 \\
0\end{array}$ & 0.00 & - & & 0.00 & 4.70 & - & $\begin{array}{c}4059 \\
.36\end{array}$ & 37.20 & 71.00 & 0.00 \\
\hline $\begin{array}{l}\text { FTWW } \\
\text { MI-28 }\end{array}$ & $\begin{array}{c}\text { Ft. } \\
\text { Wainwright }\end{array}$ & $\begin{array}{c}218.0 \\
0 \\
\end{array}$ & $\begin{array}{c}695.0 \\
0 \\
\end{array}$ & $\begin{array}{r}5392 . \\
59 \\
\end{array}$ & & . & 0.00 & - & - & $\begin{array}{c}2333 \\
.00 \\
\end{array}$ & 27.80 & 79.00 & 0.00 \\
\hline $\begin{array}{l}\text { FTWW } \\
\text { MI-29 } \\
\end{array}$ & $\begin{array}{c}\text { Ft. } \\
\text { Wainwright }\end{array}$ & $\begin{array}{c}418.4 \\
0 \\
\end{array}$ & $\begin{array}{c}371.0 \\
0 \\
\end{array}$ & 0.00 & - & & 0.00 & 4.60 & - & $\begin{array}{c}2668 \\
.17 \\
\end{array}$ & 27.50 & 63.00 & 0.00 \\
\hline $\begin{array}{l}\text { FTWW } \\
\text { MI-30 }\end{array}$ & $\begin{array}{c}\text { Ft. } \\
\text { Wainwright }\end{array}$ & $\begin{array}{c}253.7 \\
0 \\
\end{array}$ & $\begin{array}{c}600.0 \\
0 \\
\end{array}$ & 0.00 & - & - & 0.00 & - & - & $\begin{array}{c}2075 \\
.89 \\
\end{array}$ & 31.60 & 68.00 & $\begin{array}{c}403.3 \\
5 \\
\end{array}$ \\
\hline $\begin{array}{l}\text { FTWW } \\
\text { MI-30 }\end{array}$ & $\begin{array}{c}\text { Ft. } \\
\text { Wainwright }\end{array}$ & $\begin{array}{c}376.6 \\
0 \\
\end{array}$ & $\begin{array}{c}410.0 \\
0 \\
\end{array}$ & 0.00 & - & - & 0.00 & 3.50 & - & $\begin{array}{c}2493 \\
.70 \\
\end{array}$ & 27.60 & 62.00 & 0.00 \\
\hline $\begin{array}{l}\text { FTWW } \\
\text { MI-31 }\end{array}$ & $\begin{array}{c}\text { Ft. } \\
\text { Wainwright } \\
\end{array}$ & $\begin{array}{c}230.3 \\
0 \\
\end{array}$ & $\begin{array}{c}755.0 \\
0 \\
\end{array}$ & 0.00 & - & - & 0.00 & - & - & $\begin{array}{c}3040 \\
.07\end{array}$ & 31.20 & 71.00 & $\begin{array}{c}106.5 \\
4\end{array}$ \\
\hline $\begin{array}{l}\text { FTWW } \\
\text { MI-32 }\end{array}$ & $\begin{array}{c}\text { Ft. } \\
\text { Wainwright }\end{array}$ & $\begin{array}{c}886.3 \\
0\end{array}$ & $\begin{array}{c}369.0 \\
0\end{array}$ & 0.00 & - & - & 0.00 & 12.70 & - & $\begin{array}{c}3912 \\
.43\end{array}$ & 34.50 & 66.00 & 0.00 \\
\hline $\begin{array}{l}\text { FTWW } \\
\text { MI-33 } \\
\end{array}$ & $\begin{array}{c}\text { Ft. } \\
\text { Wainwright }\end{array}$ & $\begin{array}{c}332.0 \\
0 \\
\end{array}$ & $\begin{array}{c}552.0 \\
0 \\
\end{array}$ & 0.00 & - & - & 0.00 & - & - & $\begin{array}{c}3343 \\
.10 \\
\end{array}$ & 30.70 & 63.00 & 0.00 \\
\hline $\begin{array}{l}\text { FTWW } \\
\text { MI-34 }\end{array}$ & $\begin{array}{c}\text { Ft. } \\
\text { Wainwright }\end{array}$ & $\begin{array}{c}327.0 \\
0\end{array}$ & $\begin{array}{c}476.0 \\
0\end{array}$ & 0.00 & - & & 0.00 & 2.80 & - & $\begin{array}{c}3673 \\
.68\end{array}$ & 39.40 & 61.00 & 0.00 \\
\hline $\begin{array}{l}\text { FTWW } \\
\text { MI-35 }\end{array}$ & $\begin{array}{c}\text { Ft. } \\
\text { Wainwright }\end{array}$ & $\begin{array}{c}276.2 \\
0 \\
\end{array}$ & $\begin{array}{c}544.0 \\
0 \\
\end{array}$ & 0.00 & - & - & 0.00 & - & - & $\begin{array}{c}3017 \\
.12 \\
\end{array}$ & 36.00 & 66.00 & $\begin{array}{c}1126 . \\
33\end{array}$ \\
\hline $\begin{array}{l}\text { FTWW } \\
\text { MI-36 }\end{array}$ & $\begin{array}{c}\text { Ft. } \\
\text { Wainwright }\end{array}$ & $\begin{array}{c}207.8 \\
0\end{array}$ & $\begin{array}{c}350.0 \\
0\end{array}$ & 0.00 & - & - & 0.00 & - & - & $\begin{array}{c}2199 \\
.85\end{array}$ & 29.80 & 58.00 & $\begin{array}{c}479.4 \\
5\end{array}$ \\
\hline $\begin{array}{l}\text { FTWW } \\
\text { MI-37 }\end{array}$ & $\begin{array}{c}\text { Ft. } \\
\text { Wainwright }\end{array}$ & $\begin{array}{c}436.7 \\
0 \\
\end{array}$ & $\begin{array}{c}633.0 \\
0 \\
\end{array}$ & 0.00 & - & - & 0.00 & 4.10 & 72.00 & $\begin{array}{c}4224 \\
.65 \\
\end{array}$ & 33.00 & 63.00 & 0.00 \\
\hline $\begin{array}{c}\text { FTWW } \\
\text { MI-38 }\end{array}$ & $\begin{array}{c}\text { Ft. } \\
\text { Wainwright }\end{array}$ & $\begin{array}{c}380.3 \\
0\end{array}$ & $\begin{array}{c}439.0 \\
0\end{array}$ & 0.00 & - & - & 0.00 & 3.00 & - & $\begin{array}{c}2911 \\
.51\end{array}$ & 30.30 & 68.00 & $\begin{array}{c}182.6 \\
5\end{array}$ \\
\hline $\begin{array}{l}\text { FTWW } \\
\text { MI-39 }\end{array}$ & $\begin{array}{c}\text { Ft. } \\
\text { Wainwright }\end{array}$ & $\begin{array}{c}438.8 \\
0\end{array}$ & $\begin{array}{c}503.0 \\
0\end{array}$ & 0.00 & - & - & 0.00 & 4.10 & - & $\begin{array}{c}2833 \\
.46\end{array}$ & 56.10 & 71.00 & 0.00 \\
\hline $\begin{array}{l}\text { FTWW } \\
\text { MIFP01 }\end{array}$ & $\begin{array}{c}\text { Ft. } \\
\text { Wainwright }\end{array}$ & $\begin{array}{c}389.6 \\
0 \\
\end{array}$ & 68.00 & 0.00 & - & - & 0.00 & 3.70 & - & $\begin{array}{c}2429 \\
.42 \\
\end{array}$ & 47.90 & 87.00 & $\begin{array}{c}624.0 \\
5 \\
\end{array}$ \\
\hline $\begin{array}{l}\text { FTWW } \\
\text { MIFP02 }\end{array}$ & $\begin{array}{c}\text { Ft. } \\
\text { Wainwright }\end{array}$ & 19.40 & 58.00 & 0.00 & - & - & 0.00 & 3.30 & - & $\begin{array}{c}4784 \\
.79\end{array}$ & 37.30 & 69.00 & 0.00 \\
\hline $\begin{array}{l}\text { FTWW } \\
\text { MIFP03 }\end{array}$ & $\begin{array}{c}\text { Ft. } \\
\text { Wainwright }\end{array}$ & 17.70 & 63.00 & 0.00 & - & - & 0.00 & 2.80 & - & $\begin{array}{c}2810 \\
.50 \\
\end{array}$ & 34.00 & 80.00 & 0.00 \\
\hline $\begin{array}{l}\text { FTWW } \\
\text { MIFP04 }\end{array}$ & $\begin{array}{c}\text { Ft. } \\
\text { Wainwright }\end{array}$ & 18.70 & 51.00 & 0.00 & - & - & 0.00 & 3.60 & - & $\begin{array}{c}2594 \\
.71 \\
\end{array}$ & 39.70 & 74.00 & 0.00 \\
\hline $\begin{array}{l}\text { FTWW } \\
\text { MIFP05 }\end{array}$ & $\begin{array}{c}\text { Ft. } \\
\text { Wainwright }\end{array}$ & 16.30 & 52.00 & 0.00 & - & - & 0.00 & 3.80 & - & $\begin{array}{c}1602 \\
.97 \\
\end{array}$ & 35.60 & 80.00 & 0.00 \\
\hline $\begin{array}{l}\text { FTWW } \\
\text { MIFP06 }\end{array}$ & $\begin{array}{c}\text { Ft. } \\
\text { Wainwright }\end{array}$ & 14.50 & 50.00 & 0.00 & - & - & 0.00 & 2.60 & - & $\begin{array}{c}2975 \\
.79\end{array}$ & 32.10 & 77.00 & $\begin{array}{c}487.0 \\
6\end{array}$ \\
\hline $\begin{array}{l}\text { FTWW } \\
\text { MIFP07 }\end{array}$ & $\begin{array}{c}\text { Ft. } \\
\text { Wainwright }\end{array}$ & 15.90 & 92.00 & 0.00 & - & - & 0.00 & 3.40 & - & $\begin{array}{c}2773 \\
.77 \\
\end{array}$ & 36.30 & 88.00 & $\begin{array}{c}197.8 \\
7\end{array}$ \\
\hline $\begin{array}{l}\text { FTWW } \\
\text { MIFP08 }\end{array}$ & $\begin{array}{c}\text { Ft. } \\
\text { Wainwright }\end{array}$ & 31.10 & 89.00 & 0.00 & - & - & 0.00 & 9.30 & - & $\begin{array}{c}2218 \\
.22 \\
\end{array}$ & 41.90 & 83.00 & $\begin{array}{c}996.9 \\
6 \\
\end{array}$ \\
\hline $\begin{array}{l}\text { FTWW } \\
\text { MIFP09 }\end{array}$ & $\begin{array}{c}\text { Ft. } \\
\text { Wainwright }\end{array}$ & 29.20 & 83.00 & 0.00 & - & - & 0.00 & 9.30 & - & $\begin{array}{c}2153 \\
.94\end{array}$ & 40.50 & 85.00 & $\begin{array}{c}327.2 \\
5\end{array}$ \\
\hline $\begin{array}{l}\text { FTWW } \\
\text { MIFP10 } \\
\end{array}$ & $\begin{array}{c}\text { Ft. } \\
\text { Wainwright }\end{array}$ & 30.20 & 80.00 & 0.00 & - & - & 0.00 & 9.10 & - & $\begin{array}{c}3310 \\
.96 \\
\end{array}$ & 39.30 & 80.00 & 0.00 \\
\hline $\begin{array}{l}\text { FTWW } \\
\text { MIFP11 }\end{array}$ & $\begin{array}{c}\text { Ft. } \\
\text { Wainwright }\end{array}$ & 27.50 & 75.00 & 0.00 & - & . & 0.00 & 8.60 & - & $\begin{array}{c}3090 \\
.58\end{array}$ & 38.90 & 84.00 & 0.00 \\
\hline $\begin{array}{l}\text { FTWW } \\
\text { MIFP12 }\end{array}$ & $\begin{array}{c}\text { Ft. } \\
\text { Wainwright }\end{array}$ & 26.40 & 69.00 & 0.00 & - & & 0.00 & 7.60 & - & $\begin{array}{c}2273 \\
.31\end{array}$ & 39.50 & 82.00 & 0.00 \\
\hline $\begin{array}{l}\text { FTWW } \\
\text { MIFP13 }\end{array}$ & $\begin{array}{c}\text { Ft. } \\
\text { Wainwright }\end{array}$ & 22.80 & 83.00 & 0.00 & - & - & 0.00 & 6.30 & - & $\begin{array}{c}2218 \\
.22 \\
\end{array}$ & 38.90 & $\begin{array}{c}100.0 \\
0 \\
\end{array}$ & 0.00 \\
\hline $\begin{array}{l}\text { FTWW } \\
\text { MIFP14 }\end{array}$ & $\begin{array}{c}\text { Ft. } \\
\text { Wainwright }\end{array}$ & 27.10 & 68.00 & 0.00 & - & & 0.00 & 8.30 & - & $\begin{array}{c}3379 \\
.83 \\
\end{array}$ & 42.40 & 75.00 & 38.05 \\
\hline $\begin{array}{l}\text { FTWW } \\
\text { MIFP15 }\end{array}$ & $\begin{array}{c}\text { Ft. } \\
\text { Wainwright }\end{array}$ & 23.10 & 93.00 & 0.00 & - & - & 0.00 & 6.70 & - & $\begin{array}{c}3021 \\
.71\end{array}$ & 38.10 & 89.00 & 0.00 \\
\hline
\end{tabular}




\begin{tabular}{|c|c|c|c|c|c|c|c|c|c|c|c|c|c|}
\hline $\begin{array}{l}\text { FTWW } \\
\text { BCK 1b }\end{array}$ & $\begin{array}{c}\text { Ft. } \\
\text { Wainwright }\end{array}$ & 28.10 & $\begin{array}{c}318.0 \\
0\end{array}$ & 0.00 & - & - & 0.00 & - & - & $\begin{array}{c}854 . \\
58\end{array}$ & 38.20 & 89.00 & 0.00 \\
\hline KTS 01 & Kimama & 34.40 & 93.00 & 0.00 & - & - & 0.00 & - & - & $\begin{array}{c}2034 \\
.56\end{array}$ & 28.50 & 58.00 & 0.00 \\
\hline KTS 02 & Kimama & $\begin{array}{c}844.4 \\
0\end{array}$ & $\begin{array}{c}1357 . \\
00\end{array}$ & 0.00 & - & - & 0.00 & - & - & $\begin{array}{c}1047 \\
.42\end{array}$ & 52.70 & 67.00 & $\begin{array}{c}905.6 \\
3\end{array}$ \\
\hline KTS 03 & Kimama & $\begin{array}{c}956.6 \\
0\end{array}$ & $\begin{array}{c}1577 . \\
00\end{array}$ & $\begin{array}{c}14790 \\
.74\end{array}$ & - & - & 0.00 & - & - & $\begin{array}{c}638 . \\
79\end{array}$ & 34.10 & 74.00 & 0.00 \\
\hline KTS 04 & Kimama & $\begin{array}{c}481.5 \\
0\end{array}$ & $\begin{array}{c}606.0 \\
0\end{array}$ & 0.00 & - & - & 0.00 & - & - & 0.00 & 27.80 & 52.00 & 0.00 \\
\hline KTS 05 & Kimama & $\begin{array}{c}489.2 \\
0\end{array}$ & $\begin{array}{c}674.0 \\
0\end{array}$ & 0.00 & - & - & 0.00 & - & 54.00 & 0.00 & 29.20 & 61.00 & $\begin{array}{c}684.9 \\
3\end{array}$ \\
\hline KTS 06 & Kimama & $\begin{array}{c}162.5 \\
0\end{array}$ & $\begin{array}{c}118.0 \\
0\end{array}$ & 0.00 & - & - & 0.00 & - & - & $\begin{array}{c}1699 \\
.39\end{array}$ & 28.60 & 48.00 & $\begin{array}{c}1712 . \\
33\end{array}$ \\
\hline KTS 07 & Kimama & 53.50 & 73.00 & 0.00 & - & - & 0.00 & - & - & $\begin{array}{c}1460 \\
.64\end{array}$ & 24.00 & 45.00 & 0.00 \\
\hline KTS 08 & Kimama & 36.80 & $\begin{array}{c}286.0 \\
0\end{array}$ & 0.00 & - & - & 0.00 & - & - & $\begin{array}{c}1024 \\
.46\end{array}$ & 23.40 & 50.00 & 0.00 \\
\hline KTS 09 & Kimama & 30.80 & 81.00 & 0.00 & - & - & 0.00 & - & - & $\begin{array}{c}1024 \\
.46\end{array}$ & 25.00 & 52.00 & $\begin{array}{c}799.0 \\
9\end{array}$ \\
\hline KTS 10 & Kimama & $\begin{array}{c}128.7 \\
0\end{array}$ & 52.00 & 0.00 & - & - & 0.00 & - & - & $\begin{array}{c}1281 \\
.58\end{array}$ & 22.70 & 43.00 & 0.00 \\
\hline KTS 11 & Kimama & 13.10 & 62.00 & 0.00 & - & - & 0.00 & - & - & $\begin{array}{c}1612 \\
.16 \\
\end{array}$ & 26.20 & 59.00 & 0.00 \\
\hline KTS 12 & Kimama & $\begin{array}{c}494.8 \\
0 \\
\end{array}$ & $\begin{array}{c}248.0 \\
0 \\
\end{array}$ & 0.00 & - & - & 0.00 & - & - & 0.00 & 26.40 & 61.00 & 0.00 \\
\hline KTS 13 & Kimama & 84.80 & $\begin{array}{c}149.0 \\
0\end{array}$ & 0.00 & - & - & 0.00 & - & - & $\begin{array}{c}781 . \\
12\end{array}$ & 27.40 & 50.00 & 0.00 \\
\hline KTS 14 & Kimama & $\begin{array}{c}127.1 \\
0\end{array}$ & $\begin{array}{c}226.0 \\
0\end{array}$ & 0.00 & - & - & 0.00 & - & - & $\begin{array}{c}643 . \\
38\end{array}$ & 26.50 & 54.00 & 0.00 \\
\hline KTS 15 & Kimama & $\begin{array}{c}1083 . \\
60\end{array}$ & $\begin{array}{c}716.0 \\
0\end{array}$ & 0.00 & - & - & 0.00 & 4.20 & - & $\begin{array}{c}1616 \\
.75\end{array}$ & 28.00 & 66.00 & 0.00 \\
\hline KTS 16 & Kimama & $\begin{array}{c}124.1 \\
0\end{array}$ & $\begin{array}{c}237.0 \\
0\end{array}$ & 0.00 & - & - & 0.00 & - & - & 0.00 & 25.80 & 51.00 & 0.00 \\
\hline KTS 17 & Kimama & $\begin{array}{c}171.5 \\
0\end{array}$ & $\begin{array}{c}226.0 \\
0\end{array}$ & 0.00 & - & - & 0.00 & - & - & 0.00 & 25.40 & 54.00 & $\begin{array}{c}601.2 \\
2\end{array}$ \\
\hline KTS 18 & Kimama & 24.90 & 40.00 & 0.00 & - & - & 0.00 & - & - & $\begin{array}{c}2562 \\
.57\end{array}$ & 22.20 & 53.00 & $\begin{array}{c}296.8 \\
0\end{array}$ \\
\hline KTS 19 & Kimama & 9.40 & 71.00 & 0.00 & - & - & 0.00 & - & - & $\begin{array}{c}1460 \\
.64 \\
\end{array}$ & 24.30 & 42.00 & 0.00 \\
\hline KTS 20 & Kimama & 6.10 & 35.00 & 0.00 & - & - & 0.00 & - & - & $\begin{array}{c}308 . \\
21\end{array}$ & 24.40 & 48.00 & 0.00 \\
\hline KTS 21 & Kimama & - & 26.00 & 0.00 & - & - & 0.00 & - & - & $\begin{array}{c}2002 \\
.42\end{array}$ & 25.30 & 53.00 & 0.00 \\
\hline KTS 22 & Kimama & 3.90 & 37.00 & 0.00 & - & - & 0.00 & - & - & $\begin{array}{c}1837 \\
.13 \\
\end{array}$ & 26.00 & 48.00 & 45.66 \\
\hline KTS 23 & Kimama & 9.50 & 38.00 & 0.00 & - & - & 0.00 & - & - & 0.00 & 23.00 & 48.00 & 0.00 \\
\hline KTS 24 & Kimama & 7.80 & 43.00 & 0.00 & - & - & 0.00 & - & - & $\begin{array}{c}2576 \\
.34\end{array}$ & 26.10 & 49.00 & 0.00 \\
\hline KTS 25 & Kimama & $\begin{array}{c}143.3 \\
0\end{array}$ & $\begin{array}{c}108.0 \\
0\end{array}$ & 0.00 & - & - & 0.00 & - & - & $\begin{array}{c}1157 \\
.61 \\
\end{array}$ & 22.80 & 51.00 & 0.00 \\
\hline KTS 26 & Kimama & $\begin{array}{c}106.5 \\
0\end{array}$ & $\begin{array}{c}285.0 \\
0\end{array}$ & 0.00 & - & - & 0.00 & - & - & $\begin{array}{c}790 . \\
30\end{array}$ & 24.50 & 50.00 & 0.00 \\
\hline KTS 27 & Kimama & 4.90 & 32.00 & 0.00 & - & - & 0.00 & - & - & $\begin{array}{c}987 . \\
73\end{array}$ & 22.30 & 51.00 & 0.00 \\
\hline KTS 28 & Kimama & $\begin{array}{c}486.1 \\
0\end{array}$ & 72.00 & 0.00 & - & - & 0.00 & - & - & $\begin{array}{c}1497 \\
.37\end{array}$ & 25.20 & 51.00 & 0.00 \\
\hline
\end{tabular}




\begin{tabular}{|c|c|c|c|c|c|c|c|c|c|c|c|c|c|}
\hline KTS 29 & Kimama & 2.10 & 34.00 & 0.00 & - & - & $\begin{array}{r}6324 \\
1.67 \\
\end{array}$ & - & - & 0.00 & 21.30 & 47.00 & 0.00 \\
\hline KTS 30 & Kimama & - & 23.00 & 0.00 & - & - & 0.00 & - & - & $\begin{array}{c}2050 \\
.05 \\
\end{array}$ & 23.00 & 47.00 & $\begin{array}{c}190.2 \\
6 \\
\end{array}$ \\
\hline KTS 31 & Kimama & $\begin{array}{c}265.4 \\
0 \\
\end{array}$ & $\begin{array}{c}494.0 \\
0 \\
\end{array}$ & 0.00 & - & - & 0.00 & - & - & $\begin{array}{c}2677 \\
.35 \\
\end{array}$ & 23.90 & 53.00 & 0.00 \\
\hline KTS 32 & Kimama & $\begin{array}{c}187.5 \\
0 \\
\end{array}$ & $\begin{array}{c}471.0 \\
0 \\
\end{array}$ & 0.00 & - & - & 0.00 & - & - & $\begin{array}{c}1630 \\
.52 \\
\end{array}$ & 24.90 & 58.00 & $\begin{array}{c}936.0 \\
7 \\
\end{array}$ \\
\hline KTS 33 & Kimama & $\begin{array}{c}293.3 \\
0 \\
\end{array}$ & $\begin{array}{c}352.0 \\
0 \\
\end{array}$ & 0.00 & - & - & 0.00 & - & - & 7.33 & 25.00 & 51.00 & 0.00 \\
\hline KTS 34 & Kimama & $\begin{array}{c}283.3 \\
0 \\
\end{array}$ & $\begin{array}{c}315.0 \\
0 \\
\end{array}$ & 0.00 & - & - & 0.00 & - & - & $\begin{array}{c}3485 \\
.44 \\
\end{array}$ & 39.10 & 55.00 & 0.00 \\
\hline KTS 35 & Kimama & $\begin{array}{c}245.4 \\
0 \\
\end{array}$ & $\begin{array}{c}339.0 \\
0 \\
\end{array}$ & 0.00 & - & - & 0.00 & - & - & $\begin{array}{c}3646 \\
.13 \\
\end{array}$ & 29.70 & 60.00 & 0.00 \\
\hline KTS 36 & Kimama & $\begin{array}{c}274.7 \\
0 \\
\end{array}$ & $\begin{array}{c}380.0 \\
0 \\
\end{array}$ & 0.00 & - & - & 0.00 & - & - & $\begin{array}{c}978 . \\
55 \\
\end{array}$ & 26.00 & 63.00 & 0.00 \\
\hline KTS 37 & Kimama & $\begin{array}{c}262.5 \\
0 \\
\end{array}$ & $\begin{array}{c}455.0 \\
0 \\
\end{array}$ & 0.00 & - & - & 0.00 & - & - & $\begin{array}{c}1772 \\
.86 \\
\end{array}$ & 23.20 & 53.00 & 0.00 \\
\hline KTS 38 & Kimama & $\begin{array}{c}291.0 \\
0 \\
\end{array}$ & $\begin{array}{c}405.0 \\
0 \\
\end{array}$ & 0.00 & - & - & 0.00 & - & - & $\begin{array}{c}1736 \\
.12 \\
\end{array}$ & 22.90 & 58.00 & 0.00 \\
\hline KTS 39 & Kimama & $\begin{array}{c}162.4 \\
0 \\
\end{array}$ & $\begin{array}{c}297.0 \\
0 \\
\end{array}$ & 0.00 & - & - & 0.00 & - & - & $\begin{array}{c}1479 \\
.01 \\
\end{array}$ & 22.10 & 62.00 & $\begin{array}{c}327.2 \\
5 \\
\end{array}$ \\
\hline KTS 40 & Kimama & $\begin{array}{c}374.2 \\
0 \\
\end{array}$ & $\begin{array}{c}696.0 \\
0 \\
\end{array}$ & 0.00 & - & - & 0.00 & - & - & $\begin{array}{c}877 . \\
54 \\
\end{array}$ & 25.80 & 59.00 & $\begin{array}{c}1567 . \\
73 \\
\end{array}$ \\
\hline KTS 41 & Kimama & $\begin{array}{c}259.1 \\
0 \\
\end{array}$ & $\begin{array}{c}378.0 \\
0 \\
\end{array}$ & 0.00 & - & - & 0.00 & - & - & $\begin{array}{c}1745 \\
.31 \\
\end{array}$ & 24.30 & 57.00 & $\begin{array}{c}631.6 \\
6 \\
\end{array}$ \\
\hline KTS 42 & Kimama & $\begin{array}{c}222.2 \\
0 \\
\end{array}$ & $\begin{array}{c}603.0 \\
0 \\
\end{array}$ & 0.00 & - & - & 0.00 & - & - & $\begin{array}{c}928 . \\
04 \\
\end{array}$ & 24.60 & 59.00 & $\begin{array}{c}1339 . \\
42 \\
\end{array}$ \\
\hline KTS 43 & Kimama & $\begin{array}{c}313.6 \\
0\end{array}$ & $\begin{array}{c}394.0 \\
0\end{array}$ & 0.00 & - & - & 0.00 & - & - & $\begin{array}{c}1437 \\
.69\end{array}$ & 25.10 & 62.00 & 0.00 \\
\hline KTS 44 & Kimama & $\begin{array}{c}263.9 \\
0 \\
\end{array}$ & $\begin{array}{c}346.0 \\
0 \\
\end{array}$ & 0.00 & - & - & 0.00 & - & - & $\begin{array}{c}2355 \\
.96 \\
\end{array}$ & 25.40 & 52.00 & 0.00 \\
\hline KTS 45 & Kimama & $\begin{array}{c}252.3 \\
0 \\
\end{array}$ & $\begin{array}{c}434.0 \\
0 \\
\end{array}$ & 0.00 & - & - & 0.00 & - & - & $\begin{array}{c}1529 \\
.51 \\
\end{array}$ & 23.50 & 56.00 & 0.00 \\
\hline KTS 46 & Kimama & - & 22.00 & 0.00 & - & - & 0.00 & - & - & $\begin{array}{c}859 . \\
17 \\
\end{array}$ & 25.20 & 60.00 & 0.00 \\
\hline KTS 47 & Kimama & - & 22.00 & 0.00 & - & - & 0.00 & - & - & $\begin{array}{c}1373 \\
.41 \\
\end{array}$ & 24.10 & 50.00 & 0.00 \\
\hline KTS 48 & Kimama & - & 26.00 & 0.00 & - & - & 0.00 & - & - & $\begin{array}{c}1566 \\
.24 \\
\end{array}$ & 24.60 & 56.00 & $\begin{array}{c}426.1 \\
8 \\
\end{array}$ \\
\hline $\begin{array}{c}\text { MMR } \\
\text { BBG045 } \\
\text { S } \\
\end{array}$ & MMR & 11.80 & 31.00 & 0.00 & - & - & 0.00 & - & - & 0.00 & 8.10 & 15.00 & 0.00 \\
\hline $\begin{array}{c}\text { MMR } \\
\text { BFP1A } \\
\end{array}$ & MMR & 19.20 & 41.00 & 0.00 & - & - & 0.00 & - & - & $\begin{array}{c}1740 \\
.72 \\
\end{array}$ & 10.70 & 19.00 & 0.00 \\
\hline $\begin{array}{c}\text { MMR } \\
\text { BFP1B }\end{array}$ & MMR & 38.90 & 70.00 & 0.00 & - & - & 0.00 & - & - & 0.00 & 9.40 & 19.00 & $\begin{array}{c}213.0 \\
9 \\
\end{array}$ \\
\hline $\begin{array}{c}\text { MMR } \\
\text { BFP1C }\end{array}$ & MMR & 27.40 & 69.00 & 0.00 & - & - & 0.00 & - & - & $\begin{array}{c}450 . \\
54 \\
\end{array}$ & 9.60 & 13.00 & $\begin{array}{c}433.7 \\
9 \\
\end{array}$ \\
\hline $\begin{array}{c}\text { MMR } \\
\text { BFP2A }\end{array}$ & MMR & 13.70 & 35.00 & 0.00 & - & - & 0.00 & - & - & 0.00 & 9.50 & 27.00 & $\begin{array}{c}974.1 \\
2 \\
\end{array}$ \\
\hline $\begin{array}{c}\text { MMR } \\
\text { BFP2B }\end{array}$ & MMR & 26.80 & 37.00 & 0.00 & - & - & 0.00 & - & - & $\begin{array}{c}519 . \\
41 \\
\end{array}$ & 10.30 & 22.00 & 0.00 \\
\hline $\begin{array}{c}\text { MMR } \\
\text { BFP2C }\end{array}$ & MMR & 15.70 & 44.00 & 0.00 & - & - & 0.00 & - & - & $\begin{array}{c}744 . \\
39 \\
\end{array}$ & 8.90 & 23.00 & 0.00 \\
\hline $\begin{array}{c}\text { MMR } \\
\text { BFP3A }\end{array}$ & MMR & 12.10 & 35.00 & 0.00 & - & - & 0.00 & - & - & 0.00 & 9.90 & 22.00 & 0.00 \\
\hline
\end{tabular}




\begin{tabular}{|c|c|c|c|c|c|c|c|c|c|c|c|c|c|}
\hline $\begin{array}{c}\text { MMR } \\
\text { BFP3B }\end{array}$ & MMR & 17.50 & 40.00 & 0.00 & - & - & 0.00 & - & - & $\begin{array}{c}611 . \\
24 \\
\end{array}$ & 10.50 & 21.00 & 0.00 \\
\hline $\begin{array}{c}\text { MMR } \\
\text { BFP3C }\end{array}$ & MMR & 14.10 & 35.00 & 0.00 & - & - & 0.00 & - & - & $\begin{array}{c}1676 \\
.44\end{array}$ & 10.50 & 19.00 & 0.00 \\
\hline $\begin{array}{c}\text { MMR } \\
\text { BFP4A } \\
\end{array}$ & MMR & 34.10 & 46.00 & 0.00 & - & - & 0.00 & - & - & 0.00 & 8.80 & 22.00 & 38.05 \\
\hline $\begin{array}{c}\text { MMR } \\
\text { BFP4B }\end{array}$ & MMR & 25.90 & 46.00 & 0.00 & - & - & 0.00 & - & - & $\begin{array}{c}909 . \\
68\end{array}$ & 10.60 & 19.00 & $\begin{array}{c}220.7 \\
0\end{array}$ \\
\hline $\begin{array}{c}\text { MMR } \\
\text { BFP4C }\end{array}$ & MMR & 22.50 & 49.00 & 0.00 & - & - & 0.00 & - & - & $\begin{array}{c}854 . \\
58 \\
\end{array}$ & 10.10 & 20.00 & 0.00 \\
\hline $\begin{array}{c}\text { MMR } \\
\text { BFP5A } \\
\end{array}$ & MMR & 22.80 & 42.00 & 0.00 & - & - & 0.00 & - & - & $\begin{array}{c}367 . \\
90 \\
\end{array}$ & 12.20 & 20.00 & 0.00 \\
\hline $\begin{array}{c}\text { MMR } \\
\text { BFP5B }\end{array}$ & MMR & 16.50 & 33.00 & 0.00 & - & - & 0.00 & - & - & $\begin{array}{c}216 . \\
38\end{array}$ & 12.60 & 18.00 & $\begin{array}{c}273.9 \\
7 \\
\end{array}$ \\
\hline $\begin{array}{c}\text { MMR } \\
\text { BFP5C }\end{array}$ & MMR & 12.10 & 32.00 & 0.00 & - & - & 0.00 & - & - & $\begin{array}{c}1276 \\
.99 \\
\end{array}$ & 11.60 & 27.00 & 0.00 \\
\hline $\begin{array}{c}\text { MMR } \\
\text { BLB024 } \\
\text { S1 }\end{array}$ & MMR & $\begin{array}{c}274.9 \\
0\end{array}$ & $\begin{array}{c}227.0 \\
0\end{array}$ & 0.00 & $\begin{array}{c}402 . \\
90\end{array}$ & $\begin{array}{c}1157 . \\
00\end{array}$ & 0.00 & 5.80 & - & $\begin{array}{c}1231 \\
.07\end{array}$ & 28.30 & 61.00 & 0.00 \\
\hline $\begin{array}{c}\text { MMR } \\
\text { BLB027 } \\
\text { S1 }\end{array}$ & MMR & $\begin{array}{c}272.2 \\
0\end{array}$ & $\begin{array}{c}227.0 \\
0\end{array}$ & 0.00 & $\begin{array}{c}487 . \\
60\end{array}$ & $\begin{array}{c}779.0 \\
0\end{array}$ & 0.00 & 7.40 & - & $\begin{array}{c}1451 \\
.46\end{array}$ & 29.10 & 56.00 & $\begin{array}{c}700.1 \\
5\end{array}$ \\
\hline $\begin{array}{c}\text { MMR } \\
\text { BLB038 } \\
\text { S } \\
\end{array}$ & MMR & $\begin{array}{c}189.0 \\
0\end{array}$ & $\begin{array}{c}330.0 \\
0\end{array}$ & 0.00 & $\begin{array}{c}43.5 \\
0\end{array}$ & - & 0.00 & - & - & $\begin{array}{c}170 . \\
47\end{array}$ & 14.10 & 39.00 & $\begin{array}{c}989.3 \\
5\end{array}$ \\
\hline $\begin{array}{c}\text { MMR } \\
\text { BLB043 } \\
\text { S }\end{array}$ & MMR & $\begin{array}{c}1852 . \\
80\end{array}$ & $\begin{array}{c}187.0 \\
0\end{array}$ & 0.00 & $\begin{array}{c}297 . \\
10\end{array}$ & $\begin{array}{c}686.0 \\
0\end{array}$ & 0.00 & 21.20 & - & 0.00 & 37.20 & 41.00 & 0.00 \\
\hline $\begin{array}{c}\text { MMR } \\
\text { BLB047 } \\
\text { S }\end{array}$ & MMR & $\begin{array}{c}104.2 \\
0\end{array}$ & $\begin{array}{c}256.0 \\
0\end{array}$ & 0.00 & $\begin{array}{c}93.0 \\
0\end{array}$ & $\begin{array}{c}285.0 \\
0\end{array}$ & 0.00 & - & - & 0.00 & 13.80 & 53.00 & 0.00 \\
\hline $\begin{array}{c}\mathrm{MMR} \\
\mathrm{BMB} 02 \\
2 \mathrm{~S} 1\end{array}$ & MMR & $\begin{array}{c}573.3 \\
0\end{array}$ & $\begin{array}{c}648.0 \\
0\end{array}$ & 0.00 & $\begin{array}{c}1020 \\
.30\end{array}$ & $\begin{array}{c}2150 . \\
00\end{array}$ & 0.00 & 16.70 & - & $\begin{array}{c}1474 \\
.42\end{array}$ & 33.40 & $\begin{array}{c}103.0 \\
0\end{array}$ & 0.00 \\
\hline $\begin{array}{c}\text { MMR } \\
\text { BMB04 } \\
2 \mathrm{~S}\end{array}$ & MMR & $\begin{array}{c}290.4 \\
0\end{array}$ & $\begin{array}{c}342.0 \\
0\end{array}$ & 0.00 & $\begin{array}{c}155 . \\
60\end{array}$ & $\begin{array}{c}308.0 \\
0\end{array}$ & 0.00 & - & - & 0.00 & 17.10 & 42.00 & 0.00 \\
\hline $\begin{array}{c}\text { MMR } \\
\text { BMB04 } \\
4 \mathrm{~S} \\
\end{array}$ & MMR & $\begin{array}{c}1173 . \\
30\end{array}$ & $\begin{array}{c}197.0 \\
0\end{array}$ & 0.00 & $\begin{array}{c}466 . \\
70\end{array}$ & $\begin{array}{c}1238 . \\
00\end{array}$ & 0.00 & 17.80 & - & $\begin{array}{c}248 . \\
52\end{array}$ & 19.00 & 77.00 & $\begin{array}{c}365.3 \\
0\end{array}$ \\
\hline $\begin{array}{c}\text { MMR } \\
\text { BMB04 } \\
6 \mathrm{~S}\end{array}$ & MMR & $\begin{array}{c}131.6 \\
0\end{array}$ & $\begin{array}{c}287.0 \\
0\end{array}$ & 0.00 & $\begin{array}{c}367 . \\
70\end{array}$ & $\begin{array}{c}1237 . \\
00\end{array}$ & 0.00 & 2.50 & - & 0.00 & 20.40 & 78.00 & 0.00 \\
\hline $\begin{array}{c}\text { MMR } \\
\text { BMB08 } \\
2 \mathrm{~S} \\
\end{array}$ & MMR & $\begin{array}{c}179.4 \\
0\end{array}$ & $\begin{array}{c}373.0 \\
0\end{array}$ & 0.00 & $\begin{array}{c}421 . \\
90\end{array}$ & $\begin{array}{c}1030 . \\
00\end{array}$ & 0.00 & 3.80 & - & $\begin{array}{c}211 . \\
79\end{array}$ & 26.20 & 73.00 & 0.00 \\
\hline $\begin{array}{c}\text { MMR } \\
\text { BRF009 } \\
\text { S2 }\end{array}$ & MMR & 91.40 & 91.00 & 0.00 & $\begin{array}{c}81.9 \\
0\end{array}$ & - & 0.00 & 16.50 & - & $\begin{array}{c}308 . \\
21\end{array}$ & 17.40 & 36.00 & 0.00 \\
\hline $\begin{array}{c}\text { MMR } \\
\text { BRF012 } \\
\text { 3S2 }\end{array}$ & MMR & 88.90 & $\begin{array}{c}186.0 \\
0\end{array}$ & 0.00 & - & - & 0.00 & - & - & $\begin{array}{c}1786 \\
.63\end{array}$ & 14.60 & 36.00 & 0.00 \\
\hline $\begin{array}{c}\text { MMR } \\
\text { BRF012 } \\
\text { S1 }\end{array}$ & MMR & 96.60 & $\begin{array}{c}180.0 \\
0\end{array}$ & 0.00 & 2.30 & - & 0.00 & - & - & 0.00 & 19.30 & 45.00 & 0.00 \\
\hline $\begin{array}{c}\text { MMR } \\
\text { BTA020 } \\
\text { S }\end{array}$ & MMR & $\begin{array}{c}154.8 \\
0\end{array}$ & $\begin{array}{c}313.0 \\
0\end{array}$ & 0.00 & - & - & 0.00 & - & - & 0.00 & 12.00 & 30.00 & 0.00 \\
\hline
\end{tabular}




\begin{tabular}{|c|c|c|c|c|c|c|c|c|c|c|c|c|c|}
\hline $\begin{array}{c}\text { MMR } \\
\text { BTR033 } \\
\text { S1 } \\
\end{array}$ & MMR & $\begin{array}{c}221.2 \\
0\end{array}$ & $\begin{array}{c}340.0 \\
0\end{array}$ & 0.00 & $\begin{array}{c}478 . \\
00\end{array}$ & $\begin{array}{c}1210 . \\
00\end{array}$ & 0.00 & 5.80 & - & $\begin{array}{c}1254 \\
.03\end{array}$ & 25.00 & 59.00 & 0.00 \\
\hline $\begin{array}{c}\text { MMR } \\
\text { BTR034 } \\
\text { S2 } \\
\end{array}$ & MMR & $\begin{array}{c}279.5 \\
0\end{array}$ & $\begin{array}{c}515.0 \\
0\end{array}$ & 0.00 & $\begin{array}{c}483 . \\
30\end{array}$ & $\begin{array}{c}2126 . \\
00\end{array}$ & 0.00 & 6.30 & - & $\begin{array}{c}445 . \\
95\end{array}$ & 24.50 & $\begin{array}{c}120.0 \\
0\end{array}$ & $\begin{array}{c}2115 . \\
68\end{array}$ \\
\hline $\begin{array}{c}\text { MMR } \\
\text { BTR035 } \\
\text { S3 }\end{array}$ & MMR & $\begin{array}{c}215.4 \\
0\end{array}$ & $\begin{array}{c}401.0 \\
0\end{array}$ & 0.00 & $\begin{array}{c}510 . \\
60\end{array}$ & $\begin{array}{c}1734 . \\
00\end{array}$ & 0.00 & 5.80 & - & 0.00 & 25.70 & $\begin{array}{c}106.0 \\
0\end{array}$ & 0.00 \\
\hline $\begin{array}{c}\text { MMR } \\
\text { BTR037 } \\
\text { Sd }\end{array}$ & MMR & $\begin{array}{c}272.4 \\
0\end{array}$ & $\begin{array}{c}516.0 \\
0\end{array}$ & 0.00 & $\begin{array}{c}266 . \\
10\end{array}$ & $\begin{array}{c}626.0 \\
0\end{array}$ & 0.00 & 2.80 & - & $\begin{array}{c}1162 \\
.20\end{array}$ & 24.20 & 64.00 & 0.00 \\
\hline $\begin{array}{c}\text { MMR } \\
\text { BTR037 } \\
\text { Se } \\
\end{array}$ & MMR & $\begin{array}{c}475.4 \\
0\end{array}$ & $\begin{array}{c}546.0 \\
0\end{array}$ & 0.00 & $\begin{array}{c}360 . \\
10\end{array}$ & $\begin{array}{c}618.0 \\
0\end{array}$ & 0.00 & 5.40 & - & $\begin{array}{c}1474 \\
.42\end{array}$ & 26.60 & 64.00 & 0.00 \\
\hline $\begin{array}{c}\text { MMR } \\
\text { BTR037 } \\
\text { Sf } \\
\end{array}$ & MMR & $\begin{array}{c}305.0 \\
0\end{array}$ & $\begin{array}{c}551.0 \\
0\end{array}$ & 0.00 & $\begin{array}{c}277 . \\
90\end{array}$ & $\begin{array}{c}737.0 \\
0\end{array}$ & 0.00 & 2.80 & - & $\begin{array}{c}831 . \\
62\end{array}$ & 25.50 & 63.00 & $\begin{array}{c}1659 . \\
06\end{array}$ \\
\hline $\begin{array}{c}\text { MMR } \\
\text { BTR039 } \\
\text { S }\end{array}$ & MMR & $\begin{array}{c}182.5 \\
0\end{array}$ & $\begin{array}{c}483.0 \\
0\end{array}$ & 0.00 & 9.70 & - & 0.00 & - & - & $\begin{array}{c}1400 \\
.95\end{array}$ & 15.80 & 34.00 & 0.00 \\
\hline $\begin{array}{c}\text { MMR } \\
\text { BTR049 } \\
\text { B }\end{array}$ & MMR & $\begin{array}{c}256.3 \\
0\end{array}$ & $\begin{array}{c}417.0 \\
0\end{array}$ & $\begin{array}{c}96551 \\
.85\end{array}$ & $\begin{array}{c}112 . \\
90\end{array}$ & $\begin{array}{c}258.0 \\
0\end{array}$ & 0.00 & 2.70 & - & $\begin{array}{c}303 . \\
62\end{array}$ & 15.20 & 33.00 & 0.00 \\
\hline $\begin{array}{c}\text { MMR } \\
\text { BTR050 } \\
\text { B } \\
\end{array}$ & MMR & $\begin{array}{c}215.0 \\
0\end{array}$ & $\begin{array}{c}353.0 \\
0\end{array}$ & 0.00 & $\begin{array}{c}66.9 \\
0\end{array}$ & $\begin{array}{c}128.0 \\
0\end{array}$ & 0.00 & - & - & 0.00 & 13.60 & 36.00 & 0.00 \\
\hline $\begin{array}{c}\text { MMR } \\
\text { BUB078 } \\
\text { B } \\
\end{array}$ & MMR & $\begin{array}{c}106.7 \\
0\end{array}$ & 55.00 & 0.00 & $\begin{array}{c}18.8 \\
0\end{array}$ & - & 0.00 & - & - & 0.00 & 3.60 & - & $\begin{array}{c}1019 . \\
79\end{array}$ \\
\hline $\begin{array}{c}\text { MMR } \\
\text { BUB079 } \\
\text { B }\end{array}$ & MMR & 16.40 & 14.00 & 0.00 & 7.70 & - & 0.00 & - & - & $\begin{array}{c}257 . \\
70\end{array}$ & 3.40 & - & 0.00 \\
\hline $\begin{array}{c}\text { MMR } \\
\text { BUB080 } \\
\text { B } \\
\end{array}$ & MMR & 28.80 & 25.00 & 0.00 & $\begin{array}{c}14.1 \\
0\end{array}$ & - & 0.00 & - & - & 0.00 & 2.80 & 15.00 & 0.00 \\
\hline $\begin{array}{c}\text { MMR } \\
\text { BUB081 } \\
\text { B } \\
\end{array}$ & MMR & 28.50 & 45.00 & 0.00 & 6.20 & - & 0.00 & - & - & $\begin{array}{c}1301 \\
.09\end{array}$ & 4.10 & - & $\begin{array}{c}616.4 \\
4\end{array}$ \\
\hline $\begin{array}{c}\text { MMR } \\
\text { CMB00 } \\
\text { 9S2 }\end{array}$ & MMR & $\begin{array}{c}340.9 \\
0\end{array}$ & $\begin{array}{c}412.0 \\
0\end{array}$ & 0.00 & $\begin{array}{c}346 . \\
70\end{array}$ & $\begin{array}{c}1010 . \\
00\end{array}$ & 0.00 & 4.70 & - & $\begin{array}{c}1080 \\
.71\end{array}$ & 26.80 & $\begin{array}{c}111.0 \\
0\end{array}$ & 0.00 \\
\hline $\begin{array}{c}\text { MMR } \\
\text { CMB01 } \\
1 \mathrm{~S} 3 \\
\end{array}$ & MMR & $\begin{array}{c}359.0 \\
0\end{array}$ & $\begin{array}{c}353.0 \\
0\end{array}$ & 0.00 & $\begin{array}{c}399 . \\
00\end{array}$ & $\begin{array}{c}830.0 \\
0\end{array}$ & 0.00 & 5.90 & - & $\begin{array}{c}823 . \\
59\end{array}$ & 28.60 & 73.00 & 0.00 \\
\hline $\begin{array}{c}\text { MMR } \\
\text { CMB03 } \\
\text { 3B } \\
\end{array}$ & MMR & $\begin{array}{c}308.6 \\
0\end{array}$ & $\begin{array}{c}325.0 \\
0\end{array}$ & 0.00 & $\begin{array}{c}171 . \\
90\end{array}$ & $\begin{array}{c}364.0 \\
0\end{array}$ & 0.00 & 2.90 & - & $\begin{array}{c}2003 \\
.57\end{array}$ & 17.70 & 40.00 & $\begin{array}{c}129.3 \\
8\end{array}$ \\
\hline $\begin{array}{c}\text { MMR } \\
\text { CMB03 } \\
\text { 4B } \\
\end{array}$ & MMR & $\begin{array}{c}185.8 \\
0\end{array}$ & $\begin{array}{c}508.0 \\
0\end{array}$ & 0.00 & $\begin{array}{c}62.8 \\
0\end{array}$ & $\begin{array}{c}131.0 \\
0\end{array}$ & 0.00 & 2.50 & - & 0.00 & 14.00 & 27.00 & 0.00 \\
\hline $\begin{array}{c}\text { MMR } \\
\text { CMB03 } \\
\text { 5B } \\
\end{array}$ & MMR & $\begin{array}{c}154.0 \\
0\end{array}$ & $\begin{array}{c}220.0 \\
0\end{array}$ & 0.00 & 7.00 & - & 0.00 & - & - & $\begin{array}{c}910 . \\
83\end{array}$ & 12.40 & 23.00 & $\begin{array}{c}235.9 \\
2\end{array}$ \\
\hline $\begin{array}{c}\text { MMR } \\
\text { CMB03 } \\
\text { 8B }\end{array}$ & MMR & $\begin{array}{c}269.4 \\
0\end{array}$ & $\begin{array}{c}424.0 \\
0\end{array}$ & 0.00 & - & - & 0.00 & 2.90 & - & $\begin{array}{c}199 . \\
16\end{array}$ & 8.90 & 25.00 & $\begin{array}{c}601.2 \\
2\end{array}$ \\
\hline
\end{tabular}




\begin{tabular}{|c|c|c|c|c|c|c|c|c|c|c|c|c|c|}
\hline $\begin{array}{c}\text { MMR } \\
\text { CTR003 } \\
\text { S1 }\end{array}$ & MMR & $\begin{array}{c}247.1 \\
0\end{array}$ & $\begin{array}{c}831.0 \\
0\end{array}$ & 0.00 & $\begin{array}{c}116 . \\
20\end{array}$ & $\begin{array}{c}1051 . \\
00\end{array}$ & 0.00 & - & - & $\begin{array}{c}447 . \\
10\end{array}$ & 18.20 & 98.00 & 0.00 \\
\hline $\begin{array}{c}\text { MMR } \\
\text { CTR010 } \\
\text { S3 }\end{array}$ & MMR & $\begin{array}{c}300.7 \\
0\end{array}$ & $\begin{array}{c}574.0 \\
0\end{array}$ & 0.00 & $\begin{array}{c}189 . \\
60\end{array}$ & $\begin{array}{c}697.0 \\
0\end{array}$ & 0.00 & - & - & $\begin{array}{c}1521 \\
.48\end{array}$ & 23.00 & 76.00 & 0.00 \\
\hline $\begin{array}{c}\text { MMR } \\
\text { CTR012 } \\
\text { B } \\
\end{array}$ & MMR & $\begin{array}{c}321.2 \\
0\end{array}$ & $\begin{array}{c}570.0 \\
0\end{array}$ & 0.00 & $\begin{array}{c}65.3 \\
0\end{array}$ & $\begin{array}{c}188.0 \\
0\end{array}$ & 0.00 & - & - & 0.00 & 18.90 & 46.00 & 0.00 \\
\hline $\begin{array}{c}\text { MMR } \\
\text { CTR013 } \\
\text { B } \\
\end{array}$ & MMR & $\begin{array}{c}189.5 \\
0\end{array}$ & $\begin{array}{c}344.0 \\
0\end{array}$ & 0.00 & $\begin{array}{c}11.1 \\
0\end{array}$ & - & 0.00 & - & - & 0.00 & 11.20 & 24.00 & 0.00 \\
\hline $\begin{array}{c}\text { MMR } \\
\text { CTR014 } \\
\text { B }\end{array}$ & MMR & $\begin{array}{c}269.7 \\
0\end{array}$ & $\begin{array}{c}268.0 \\
0\end{array}$ & 0.00 & - & - & 0.00 & - & - & $\begin{array}{c}506 . \\
79\end{array}$ & 7.50 & 15.00 & 0.00 \\
\hline $\begin{array}{c}\text { MMR } \\
\text { CTR015 } \\
\text { B }\end{array}$ & MMR & 45.50 & 50.00 & 0.00 & - & - & 0.00 & - & - & $\begin{array}{c}988 . \\
88\end{array}$ & 4.10 & 10.00 & 0.00 \\
\hline $\begin{array}{c}\text { MMR } \\
\text { CTR017 } \\
\text { B }\end{array}$ & MMR & 5.20 & 16.00 & $\begin{array}{l}\text { could } \\
\text { not } \\
\text { make } \\
\text { pellet }\end{array}$ & - & - & & - & - & & 3.40 & - & \\
\hline $\begin{array}{c}\text { MMR } \\
\text { CUB019 } \\
\text { S1 } \\
\end{array}$ & MMR & $\begin{array}{c}824.1 \\
0\end{array}$ & $\begin{array}{c}323.0 \\
0\end{array}$ & 0.00 & $\begin{array}{c}168 . \\
60\end{array}$ & $\begin{array}{c}446.0 \\
0\end{array}$ & 0.00 & 9.10 & - & $\begin{array}{c}773 . \\
09\end{array}$ & 17.20 & 51.00 & 0.00 \\
\hline $\begin{array}{c}\text { MMR } \\
\text { CUB020 } \\
\text { S2 }\end{array}$ & MMR & $\begin{array}{c}245.3 \\
0\end{array}$ & $\begin{array}{c}515.0 \\
0\end{array}$ & 0.00 & $\begin{array}{c}114 . \\
40\end{array}$ & $\begin{array}{c}352.0 \\
0\end{array}$ & 0.00 & - & - & $\begin{array}{c}38.4 \\
7\end{array}$ & 16.30 & 46.00 & 0.00 \\
\hline $\begin{array}{c}\text { MMR } \\
\text { E1LBFP }\end{array}$ & MMR & 41.90 & 70.00 & 0.00 & - & - & 0.00 & - & - & $\begin{array}{c}878 . \\
69 \\
\end{array}$ & 10.50 & 20.00 & 0.00 \\
\hline $\begin{array}{c}\text { MMR } \\
\text { E2 } \\
\end{array}$ & MMR & 6.90 & 18.00 & 0.00 & - & - & 0.00 & - & - & $\begin{array}{c}167 . \\
02 \\
\end{array}$ & 10.30 & 17.00 & 0.00 \\
\hline $\begin{array}{c}\text { MMR } \\
\text { E3 }\end{array}$ & MMR & 17.30 & 35.00 & 0.00 & - & - & 0.00 & - & - & $\begin{array}{c}134 . \\
89\end{array}$ & 6.90 & 19.00 & 0.00 \\
\hline $\begin{array}{c}\text { MMR } \\
\text { E4 }\end{array}$ & MMR & - & 8.00 & 0.00 & - & - & 0.00 & - & - & 0.00 & 7.30 & 14.00 & 0.00 \\
\hline $\begin{array}{c}\text { MMR } \\
\text { E5 }\end{array}$ & MMR & - & - & 0.00 & - & - & 0.00 & - & - & $\begin{array}{c}378 . \\
23\end{array}$ & 7.60 & 10.00 & $\begin{array}{c}768.6 \\
5 \\
\end{array}$ \\
\hline $\begin{array}{c}\text { MMR } \\
\text { IBB004 } \\
\text { S1 }\end{array}$ & MMR & $\begin{array}{c}1038 . \\
70\end{array}$ & $\begin{array}{c}560.0 \\
0\end{array}$ & 0.00 & $\begin{array}{c}67.0 \\
0\end{array}$ & $\begin{array}{c}826.0 \\
0\end{array}$ & 0.00 & 9.90 & - & 0.00 & 33.90 & $\begin{array}{c}130.0 \\
0\end{array}$ & 0.00 \\
\hline $\begin{array}{c}\text { MMR } \\
\text { IBB018 } \\
\text { S2 }\end{array}$ & MMR & $\begin{array}{c}515.3 \\
0\end{array}$ & $\begin{array}{c}1212 . \\
00\end{array}$ & $\begin{array}{c}15624 \\
.07\end{array}$ & $\begin{array}{c}223 . \\
70\end{array}$ & $\begin{array}{c}203.0 \\
0\end{array}$ & 0.00 & 3.60 & - & $\begin{array}{c}56.8 \\
3\end{array}$ & 21.60 & 54.00 & 0.00 \\
\hline $\begin{array}{c}\text { MMR } \\
\text { IFP- } \\
\text { TA001S } \\
1\end{array}$ & MMR & $\begin{array}{c}102.3 \\
0\end{array}$ & $\begin{array}{c}151.0 \\
0\end{array}$ & $\begin{array}{l}\text { sampl } \\
\text { e not } \\
\text { found }\end{array}$ & - & - & & - & - & & 13.60 & 26.00 & \\
\hline $\begin{array}{c}\text { MMR } \\
\text { IFP- } \\
\text { TA014S } \\
2\end{array}$ & MMR & $\begin{array}{c}305.5 \\
0\end{array}$ & $\begin{array}{c}348.0 \\
0\end{array}$ & 0.00 & - & - & 0.00 & - & - & $\begin{array}{c}157 . \\
84\end{array}$ & 13.80 & 23.00 & 0.00 \\
\hline $\begin{array}{c}\text { MMR } \\
\text { ILB003S } \\
3 \\
\end{array}$ & MMR & $\begin{array}{c}108.5 \\
0\end{array}$ & $\begin{array}{c}108.0 \\
0\end{array}$ & 0.00 & $\begin{array}{c}285 . \\
30\end{array}$ & $\begin{array}{c}509.0 \\
0\end{array}$ & 0.00 & 5.90 & - & $\begin{array}{c}1434 \\
.24\end{array}$ & $\begin{array}{c}985.8 \\
0\end{array}$ & 49.00 & 0.00 \\
\hline $\begin{array}{c}\text { MMR } \\
\text { ITR002S } \\
1\end{array}$ & MMR & $\begin{array}{c}341.9 \\
0\end{array}$ & $\begin{array}{c}469.0 \\
0\end{array}$ & 0.00 & $\begin{array}{c}97.4 \\
0\end{array}$ & $\begin{array}{c}632.0 \\
0\end{array}$ & 0.00 & - & - & $\begin{array}{c}456 . \\
28\end{array}$ & 19.60 & 62.00 & 0.00 \\
\hline
\end{tabular}




\begin{tabular}{|c|c|c|c|c|c|c|c|c|c|c|c|c|c|}
\hline $\begin{array}{c}\text { MMR } \\
\text { ITR009S } \\
2\end{array}$ & MMR & $\begin{array}{c}632.3 \\
0\end{array}$ & $\begin{array}{c}442.0 \\
0\end{array}$ & 0.00 & $\begin{array}{c}106 . \\
30\end{array}$ & $\begin{array}{c}504.0 \\
0\end{array}$ & 0.00 & 4.00 & - & 0.00 & 14.20 & 60.00 & 0.00 \\
\hline $\begin{array}{c}\text { MMR } \\
\text { ITR013S } \\
3\end{array}$ & MMR & $\begin{array}{c}328.6 \\
0\end{array}$ & $\begin{array}{c}444.0 \\
0\end{array}$ & 0.00 & $\begin{array}{c}86.7 \\
0\end{array}$ & $\begin{array}{c}684.0 \\
0\end{array}$ & 0.00 & 2.00 & - & 0.00 & 16.60 & 79.00 & 0.00 \\
\hline $\begin{array}{c}\text { MMR } \\
\text { IUB010 } \\
\text { S1 }\end{array}$ & MMR & $\begin{array}{c}395.7 \\
0\end{array}$ & $\begin{array}{c}213.0 \\
0\end{array}$ & 0.00 & 3.30 & - & 0.00 & - & - & $\begin{array}{c}111 . \\
93\end{array}$ & 14.00 & 35.00 & 0.00 \\
\hline $\begin{array}{c}\text { MMR } \\
\text { IUB014 } \\
\text { B } \\
\end{array}$ & MMR & 36.00 & 87.00 & 0.00 & $\begin{array}{c}19.7 \\
0\end{array}$ & - & 0.00 & - & - & $\begin{array}{c}1117 \\
.44\end{array}$ & 7.40 & 18.00 & 0.00 \\
\hline $\begin{array}{c}\text { MMR } \\
\text { IUB015 } \\
\text { B } \\
\end{array}$ & MMR & 30.20 & 30.00 & 0.00 & 3.80 & - & 0.00 & - & - & $\begin{array}{c}1590 \\
.35\end{array}$ & 9.30 & 12.00 & 0.00 \\
\hline $\begin{array}{c}\text { MMR } \\
\text { IUB017 } \\
\text { B }\end{array}$ & MMR & $\begin{array}{c}186.6 \\
0\end{array}$ & $\begin{array}{c}230.0 \\
0\end{array}$ & 0.00 & - & - & 0.00 & - & - & $\begin{array}{c}437 . \\
92\end{array}$ & 7.10 & 14.00 & $\begin{array}{c}410.9 \\
6\end{array}$ \\
\hline MMR J1 & MMR & 34.90 & 96.00 & 0.00 & - & - & 0.00 & - & - & $\begin{array}{c}24.6 \\
9\end{array}$ & 31.60 & 76.00 & 0.00 \\
\hline MMR J3 & MMR & 3.00 & 13.00 & 0.00 & - & - & 0.00 & - & - & $\begin{array}{c}29.2 \\
8\end{array}$ & 22.80 & 35.00 & 0.00 \\
\hline MMR J4 & MMR & - & 17.00 & 0.00 & - & - & 0.00 & - & - & $\begin{array}{c}1897 \\
.97 \\
\end{array}$ & 12.70 & 30.00 & 0.00 \\
\hline MMR J6 & MMR & 84.40 & $\begin{array}{c}124.0 \\
0 \\
\end{array}$ & 0.00 & - & - & 0.00 & - & - & $\begin{array}{c}819 . \\
00 \\
\end{array}$ & 11.90 & 25.00 & $\begin{array}{c}958.9 \\
0 \\
\end{array}$ \\
\hline $\begin{array}{c}\text { MMR } \\
\text { K2RBFP } \\
\end{array}$ & MMR & 12.30 & 31.00 & 0.00 & - & - & 0.00 & - & - & $\begin{array}{c}1608 \\
.71 \\
\end{array}$ & 16.30 & 33.00 & 0.00 \\
\hline $\begin{array}{c}\text { MMR } \\
\text { K2RBFP }\end{array}$ & MMR & 11.70 & 35.00 & 0.00 & - & - & 0.00 & - & - & $\begin{array}{c}1608 \\
.71\end{array}$ & 17.00 & 42.00 & 0.00 \\
\hline $\begin{array}{c}\text { MMR } \\
\text { K3 } \\
\end{array}$ & MMR & - & 22.00 & 0.00 & - & - & 0.00 & - & - & $\begin{array}{c}2416 \\
.80 \\
\end{array}$ & 11.30 & 26.00 & 0.00 \\
\hline $\begin{array}{c}\text { MMR } \\
\text { K5 } \\
\end{array}$ & MMR & - & 20.00 & 0.00 & - & - & 0.00 & - & - & $\begin{array}{c}819 . \\
00 \\
\end{array}$ & 9.60 & 26.00 & 0.00 \\
\hline $\begin{array}{c}\text { MMR } \\
\text { KILBFP }\end{array}$ & MMR & $\begin{array}{c}1938 . \\
70 \\
\end{array}$ & 55.00 & 0.00 & $\begin{array}{c}412 . \\
80 \\
\end{array}$ & - & 0.00 & 18.50 & - & $\begin{array}{c}29.2 \\
8\end{array}$ & 17.30 & 62.00 & 0.00 \\
\hline
\end{tabular}

Denotes that the concentration is below detection limit of the instrument 


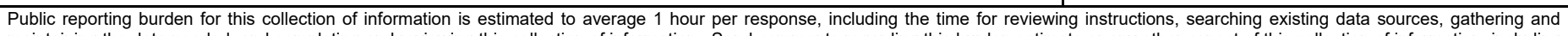

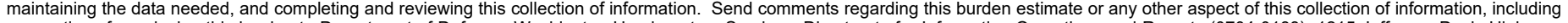

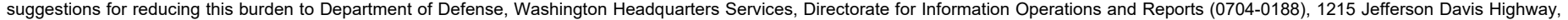

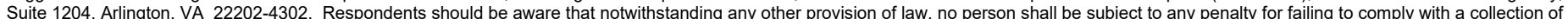
information if it does not display a currently valid OMB control number. PLEASE DO NOT RETURN YOUR FORM TO THE ABOVE ADDRESS.

\begin{tabular}{l|l|l} 
1. REPORT DATE (DD-MM-YYYY) & 2. REPORT TYPE & 3. DATES COVERED (FrOm - TO)
\end{tabular}

\begin{tabular}{l|l|l} 
June 2021 & Final \\
\hline
\end{tabular}

\section{TITLE AND SUBTITLE}

Application of Laser Induced Breakdown Spectroscopy (LIBS) for Environmental, Chemical, and Biological Sensing

\section{5a. CONTRACT NUMBER}

5b. GRANT NUMBER

5c. PROGRAM ELEMENT NUMBER $633734 \mathrm{~A}$

\section{AUTHOR(S)}

5d. PROJECT NUMBER

Holly VerMeulen, Jay L. Clausen, Ashley Mossell, Michael Morgan, Komi Messan, and Sam Beal

T15

5e. TASK NUMBER

5f. WORK UNIT NUMBER

02

7. PERFORMING ORGANIZATION NAME(S) AND ADDRESS(ES)

U.S. Army Engineer Research and Development Center

8. PERFORMING ORGANIZATION REPORT NUMBER

Cold Regions Research and Engineering Laboratory

72 Lyme Road

ERDC CRREL MP-21-2

Hanover, NH 03755

9. SPONSORING / MONITORING AGENCY NAME(S) AND ADDRESS(ES)

Headquarters, U.S. Army Corps of Engineers

Washington, DC 20314-1000

\section{DISTRIBUTION / AVAILABILITY STATEMENT}

Approved for public release; distribution is unlimited.

\section{SUPPLEMENTARY NOTES}

This paper was originally presented at the SPIE 2019 Conference held in San Diego, CA on 11-15 August 2019 and published in the conference proceedings.

\section{ABSTRACT}

The Army is interested in sensors capable of characterizing/monitoring the environment (battlefield or military training ranges) at proximal distances. Recently, we evaluated laser induced breakdown spectroscopy (LIBS) systems (hand-held, proximal, and bench top) for the characterization of metals (antimony, copper, lead, tungsten, and zinc) in soils obtained from military training ranges. We then compared the results to findings obtained with standard field and laboratory instrumentation for metals analysis - X-ray Fluorescence (XRF) and Inductively Couple Plasma- Optical Emission Spectroscopy (ICP-OES).

\section{SUBJECT TERMS}

Laser induced breakdown spectroscopy (LIBS), inductively coupled plasma- optical emission spectroscopy (ICP-OES),

$\mathrm{X}$-ray fluorescence (XRF)

\section{SECURITY CLASSIFICATION OF:}

\section{a. REPORT}

Unclassified

\section{b. ABSTRACT}

Unclassified c. THIS PAGE

Unclassified
17. LIMITATION OF ABSTRACT

SAR
18. NUMBER OF PAGES

28 19a. NAME OF RESPONSIBLE PERSON

19b. TELEPHONE NUMBER (include area code) 